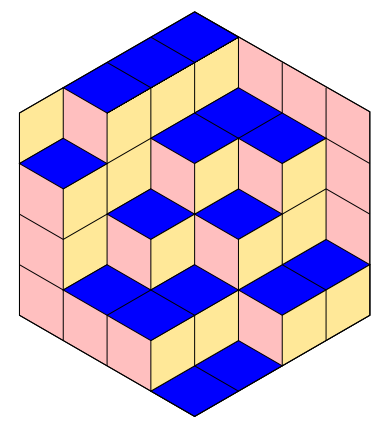

Mark Blume \& Lutz Hille

Quivers and moduli spaces of pointed curves of genus zero

Volume 4, issue 1 (2021), p. 89-124.

<http://alco.centre-mersenne.org/item/ALCO_2021__4_1_89_0>

(c) The journal and the authors, 2021.

Some rights reserved.

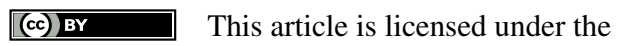

Creative Commons ATtribution 4.0 International LiCEnSE.

http://creativecommons.org/licenses/by/4.0/

Access to articles published by the journal Algebraic Combinatorics on the website http://alco.centre-mersenne.org/ implies agreement with the Terms of Use (http://alco.centre-mersenne.org/legal/).

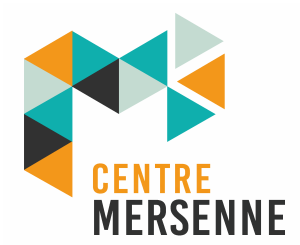

Algebraic Combinatorics is member of the Centre Mersenne for Open Scientific Publishing www.centre-mersenne.org 


\title{
Quivers and moduli spaces of pointed curves of genus zero
}

\author{
Mark Blume \& Lutz Hille
}

\begin{abstract}
We construct moduli spaces of representations of quivers over arbitrary schemes and show how moduli spaces of pointed curves of genus zero like the Grothendieck-Knudsen moduli spaces $\bar{M}_{0, n}$ and the Losev-Manin moduli spaces $\bar{L}_{n}$ can be interpreted as inverse limits of moduli spaces of representations of certain bipartite quivers. We also investigate the case of more general Hassett moduli spaces $\bar{M}_{0, a}$ of weighted pointed stable curves of genus zero.
\end{abstract}

\section{INTRODUCTION}

The main topic of this paper is the relation of moduli spaces of pointed curves of genus zero, in particular the Grothendieck-Knudsen moduli spaces $\bar{M}_{0, n}$ and the LosevManin moduli spaces $\bar{L}_{n}$, but also more general Hassett moduli spaces of weighted pointed stable curves of genus zero $\bar{M}_{0, a}$, to moduli spaces of representations of the following quivers $Q_{n}$ and $P_{n}$ with fixed dimension vector as indicated by the numbers at the vertices:

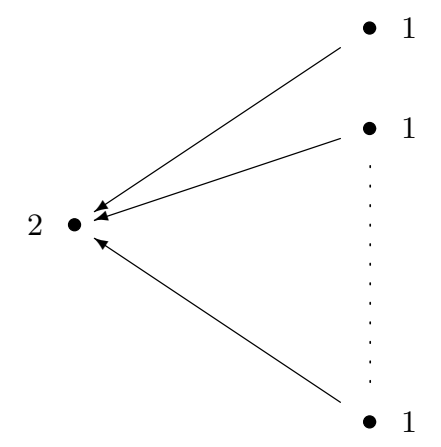

$Q_{n}$

$(n+1$ vertices $)$

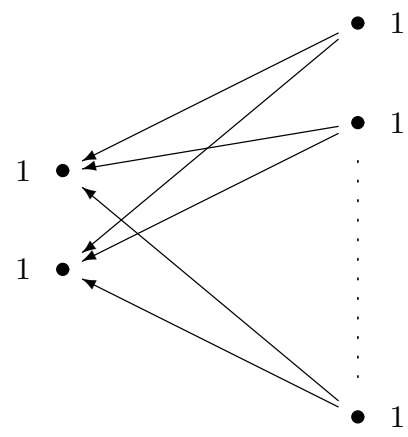

$P_{n}$

$(n+2$ vertices $)$

Manuscript received 6th February 2019, revised 4th March 2020, accepted 14th September 2020.

KEYWORDS. Moduli spaces, quiver representations, geometric invariant theory, algebraic stacks, root systems.

ACKnowledGements. Research supported by DFG CRC 878 Groups, Geometry and Actions. 
Moduli spaces of representations $\mathcal{M}^{\theta}(Q)$ of a quiver $Q$ with some fixed dimension vector are constructed via geometric invariant theory (GIT) and depend on the choice of a weight $\theta$. The collection of $\mathcal{M}^{\theta}(Q)$ for $\theta$ representing the finitely many GIT equivalence classes forms an inverse system and as inverse limit we have a space $\lim _{\theta} \mathcal{M}^{\theta}(Q)$ independent of choice of weights.

In this paper we show over arbitrary base schemes, see theorems 3.16 and 3.17 (over $\mathbb{C}$ this is essentially known via different methods, though usually formulated without quivers, see below):

THEOREM.

$$
\bar{L}_{n} \cong \lim _{\theta} \mathcal{M}^{\theta}\left(P_{n}\right) \quad \bar{M}_{0, n} \cong \underbrace{\lim }_{\theta} \mathcal{M}^{\theta}\left(Q_{n}\right)
$$

Our methods also apply to more general Hassett moduli spaces $\bar{M}_{0, a}$ of weighted pointed stable curves of genus zero [15]. In theorem 4.4 we show that any of these Hassett moduli spaces is a limit of GIT quotients over an area in the weight space (considering normalised weights), which is new already over $\mathbb{C}$ :

THEOREM.

$$
\bar{M}_{0, a} \cong \lim _{\overleftarrow{\theta<a}} \mathcal{M}^{\theta}\left(Q_{n}\right)
$$

It is natural to work over Spec $\mathbb{Z}$ as the moduli spaces of pointed curves are defined over the integers, and so are moduli spaces of representations of quivers. However, we have to extend the results on moduli spaces of representations of quivers in [20] to arbitrary base schemes (see theorem 1.8):

THEOREM. For a finite quiver $Q$ with indivisible dimension vector and generic weight $\theta$ there is, over an arbitrary base scheme, a fine moduli space $\mathcal{M}^{\theta}(Q)$ whose $Y$-valued points correspond to $\theta$-stable representations over $Y$ up to isomorphism over a Zariski cover.

Here the main issue is less the construction of these spaces by application of the theory of GIT over arbitrary schemes [1], [29] to this problem, but more the proof that these GIT quotients for generic weights are in fact fine moduli spaces of quiver representations and the application of the necessary techniques, like the result of $[2]$ in the proof of proposition 1.7.

To show the isomorphisms between moduli spaces of pointed curves of genus 0 and limits of GIT quotients we use the interpretation of these GIT quotients for generic weights as fine moduli spaces and compare the functor of the limit to the moduli functor of pointed curves via the following steps:

- The functor of the limit associates to a scheme $Y$ the set of collections $\left(\varphi_{\theta}\right)_{\theta}$ of morphisms (or equivalently collections $\left(V^{\theta}\right)_{\theta}$ of equivalence classes of $\theta$-stable representations over $Y$ ) for generic $\theta$ (i.e. corresponding to full-dimensional GIT equivalence classes) that satisfy relations coming from GIT classes of codimension one, see proposition 1.16 and (7) at the beginning of subsection 2.4.

- In proposition 2.15 we express the relations between the representations $\left(V^{\theta}\right)_{\theta}$ in the limit functor by certain equations. Whereas the original relations are local in the weight space, we have equations relating pairs of representations for all generic $\theta$. We can reduce these sets of equations by remark 2.17 .

- In the proofs of the results for the individual moduli spaces of pointed curves (theorems 3.16, 3.17 and 4.4) we restrict the collections $\left(V^{\theta}\right)_{\theta}$ to certain sets of generic weights. Considering representations as $\mathrm{P}^{1}$-bundles with $n$ sections (see corollary 2.10 and 2.12 ), these weights $\theta^{i}$ (resp. $\theta^{T}$ ) correspond to sets of 
three sections, such that a representation is stable with respect to this weight if and only if these three sections are disjoint.

- Normalising the representations with respect to the three sections for each of these weights, the functor of the inverse limit becomes a functor consisting of sections of $\mathbb{P}^{1}$ that satisfy certain equations. We compare these functors of the limit with the functors of the moduli spaces of pointed curves, described in theorems $3.11,3.14$ and 4.3, that arise from a natural closed embedding of these moduli spaces into a products of $\mathrm{P}^{1}$ 's related to root systems of type $A$.

There is the following known relation between moduli spaces of pointed curves of genus 0 and GIT quotients of $\left(\mathbb{P}^{1}\right)^{n}$. Working over the complex numbers, by [19] under some assumptions limits of GIT quotients of toric varieties $(X, T)$ by subtori of $T$ are the same as Chow quotients. Toric Chow quotients can be described by fibre polytopes, and in the case of quotients of projective spaces by secondary polytopes $[19,9]$. The Losev-Manin moduli space $\bar{L}_{n}$ is the projective toric variety corresponding to the $(n-1)$-dimensional permutohedron [23], which arises as secondary polytope of the product of simplices $\Delta^{1} \times \Delta^{n-1}$ and as fibre polytope for the operation of $\mathbb{G}_{m}$ on $\left(\mathbb{P}^{1}\right)^{n}$. By the Gelfand-MacPherson correspondence (see [17]) the GIT quotients $\left(\mathbb{P}^{1}\right)^{n}$ by $\mathrm{PGL}(2)$ and the GIT quotients of the Grassmannian $G(2, n)$ by the maximal torus $\left(\mathbb{G}_{m}\right)^{n} \subset \mathrm{GL}(n)$, and similarly the Chow quotients $\left(\mathbb{P}^{1}\right)^{n} / / \operatorname{PGL}(2)$ and $G(2, n) / /\left(\mathbb{G}_{m}\right)^{n}$ coincide over the complex numbers. By [17] the Chow quotients $G(2, n) / /\left(\mathbb{G}_{m}\right)^{n}$ coincide with the Hilbert quotients $G(2, n) / / /\left(\mathbb{G}_{m}\right)^{n}$ and by [18] $\bar{M}_{0, n}$ is the closure of the space of Veronese curves in the Hilbert scheme or Chow variety. This is used to show that the Chow quotient $G(2, n) / /\left(\mathbb{G}_{m}\right)^{n}$ is isomorphic to $\bar{M}_{0, n}$, see $[17,(4.1 .8)]$. Together the works [17], [18], [19] show that over the complex numbers $\bar{M}_{0, n}$ is the limit of GIT quotients $\left(\mathbb{P}^{1}\right)^{n} /{ }_{\theta} \operatorname{PGL}(2)$ (see also [32]).

With the straightforward link between the moduli spaces of representations $\mathcal{M}^{\theta}\left(Q_{n}\right), \mathcal{M}^{\theta}\left(P_{n}\right)$ and the GIT quotients $\left(\mathbb{P}^{1}\right)^{n} /{ }_{\theta} \operatorname{PGL}(2),\left(\mathbb{P}^{1}\right)^{n} /{ }_{\theta} \mathbb{G}_{m}$ as described in subsection 2.3 (related to the Gelfand-MacPherson correspondence) we recover the results that over the complex numbers $\bar{L}_{n} \cong \lim _{\theta}\left(\mathbb{P}^{1}\right)^{n} /{ }_{\theta} \mathbb{G}_{m}([19,23])$ and $\bar{M}_{0, n} \cong \lim _{\theta}\left(\mathbb{P}^{1}\right)^{n} /_{\theta} \mathrm{PGL}(2)([17])$. Our different, more direct methods are valid over arbitrary base schemes and do not involve Chow varieties.

It is known by [11] that over the complex numbers for fixed $n$ all Hassett moduli spaces of $n$-pointed weighted stable curves of genus zero can be realised as GIT quotients of a certain space involving the component of the Chow variety of degree $d$ curves in $\mathbb{P}^{d}$ containing rational normal curves, which, as a parameter space of embedded $n$-pointed curves of genus zero, is already close to the moduli problem.

In the present paper we describe these Hassett moduli spaces as a limit of GIT quotients that come from quivers, hence in particular GIT quotients of affine space. Here the individual GIT quotients are quite simple, furthermore, in our construction we restrict to a collection of GIT quotients for generic weights all of which are products of $\mathrm{P}^{1}$ 's.

Observing that the limits of moduli spaces of representations for these very simple quivers give rise to relevant moduli spaces of pointed curves, it may be interesting to describe and identify the varieties that arise this way from other quivers.

OUtLINE OF THE PAPER. In section 1 we construct fine moduli spaces of quiver representations over arbitrary schemes. To construct the spaces $\mathcal{M}^{\theta-s}(Q, d) \subseteq \mathcal{M}^{\theta}(Q, d)$ of $\theta$-stable and $\theta$-semistable representations we apply the theory of GIT (see [24]) over arbitrary base schemes, using the formulation in terms of stacks in [1]. We show in theorem 1.8 that for a finite quiver $Q$, an indivisible dimension vector $d$ and a weight $\theta$ the space $\mathcal{M}^{\theta-s}(Q, d)$ is a fine moduli space parametrising $\theta$-stable representations 
up to isomorphism over a Zariski cover. The main point here is to show that the natural group operation on the stable locus of the representation space is free and the morphism to the quotient a torsor, see proposition 1.7. This extends the results of $[20]$.

The first step to describe the functors of points of inverse limits of moduli spaces of quiver representations is taken in proposition 1.16 by describing the functor of the limit by the functors of the moduli spaces $\mathcal{M}^{\theta}(Q, d)$ for equivalence classes of generic weights $\theta$ subject to some relations.

Section 2 contains on the one hand some preparations and miscellaneous material concerning the quivers $P_{n}$ and $Q_{n}$ : the structure of the weight spaces for the quivers $P_{n}$ and $Q_{n}$ (compare to $[17,(1.2),(1.3)],[9,7.3 . \mathrm{C}]$ ); the relation between $Q_{n+2}$ and $P_{n}$, their weights and stable representations (this corresponds to the morphism $\bar{M}_{0, n+2} \rightarrow \bar{L}_{n}$ after a choice of two of the $n+2$ marked points); the relation of moduli spaces of representations for $P_{n}$ and $Q_{n}$ and GIT quotients of $\left(\mathbb{P}^{1}\right)^{n}$. The last two points, discussed in subsections 2.2 and 2.3 , both derive from different presentations of certain stacks.

On the other hand subsection 2.4 is one of the central parts of this paper where in proposition 2.15 we describe the functor of the limit of moduli spaces of representations for the quivers $P_{n}$ and $Q_{n}$ in terms of families of representations $\left(V^{\theta}\right)_{\theta}$, where the weights $\theta$ represent the equivalence classes of generic weights, satisfying certain equations.

In section 3 we consider the Grothendieck-Knudsen moduli spaces and LosevManin moduli spaces. It is known that the moduli spaces $\bar{L}_{n}$ and $\bar{M}_{0, n}$ can be embedded into a product of $\mathbb{P}^{1}$ 's via the natural morphisms $\bar{L}_{n} \rightarrow \bar{L}_{2} \cong \mathbb{P}^{1}$ and $\bar{M}_{0, n} \rightarrow \bar{M}_{0,4} \cong \mathbb{P}^{1}$ (see $\left.[3,10]\right)$. These closed embeddings have interpretations in terms of root systems of type $A$. In the case of the Losev-Manin moduli space $\bar{L}_{n} \subseteq\left(\mathbb{P}^{1}\right)^{\left(\begin{array}{l}n \\ 2\end{array}\right)}$ each copy of $\mathbb{P}^{1}$ corresponds to a root subsystem in $A_{n-1}$ isomorphic to $A_{1}$, whereas the equations come from the root subsystems isomorphic to $A_{2}$, see [3].

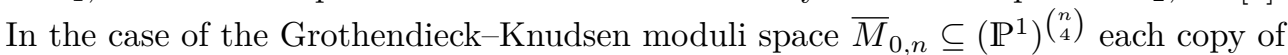
$\mathrm{P}^{1}$ corresponds to a root subsystem in $A_{n-1}$ isomorphic to $A_{3}$, whereas the equations come from the root subsystems isomorphic to $A_{4}$. This way the moduli space $\bar{M}_{0, n}$ can also be interpreted as the cross-ratio variety of root systems of type $A_{3}$ in $A_{n-1}$, cf. [27, 28]. We summarise the results on these embeddings, the interpretation in terms of root systems and the related description of the functor of points of $\bar{L}_{n}$ and $\bar{M}_{0, n}$ in theorems 3.11 and 3.14 .

Proving that there are isomorphisms $\bar{L}_{n} \cong \lim _{\theta} \mathcal{M}^{\theta}\left(P_{n}\right)$ (theorem 3.16) and $\bar{M}_{0, n} \cong \lim _{\theta} \mathcal{M}^{\theta}\left(Q_{n}\right)$ (theorem 3.17), we compare the moduli functors of pointed curves with the corresponding functors of the limit. We use the description of proposition 2.15 and simplify it, where the main step consists of restricting the family of representations $\left(V^{\theta}\right)_{\theta}$ to certain sets of weights of the form $\theta^{i}$ (resp. $\theta^{T}$ ).

In section 4 we apply the same methods to the Hassett moduli spaces to show that $\bar{M}_{0, a} \cong \lim _{\theta<a} \mathcal{M}^{\theta}\left(Q_{n}\right)$ (theorem 4.4). Here some additions are necessary to take into account the notion of $a$-stability of $n$-pointed curves. On the quiver side this corresponds to a restriction to weights in the convex polytope $P(a)=\{\theta \in \Delta(2, n) \mid \theta<a\}$. We also need a description of the moduli functor $\bar{M}_{0, a}$ analogous to that of $\bar{L}_{n}$ and $\bar{M}_{0, n}$ arising from an embedding into a product of $\mathbb{P}^{1}$ 's, see theorem 4.3 .

Notations And COnventions. We work over a base scheme $S$, usually we think of $S$ as Spec $\mathbb{Z}$. Products are over the base scheme $S$ unless otherwise specified. We use the theory of schemes as developed in $[12,13]$. We sometimes write $y \in Y$ for 
a geometric point $y:$ Spec $k \rightarrow Y$. We use the standard theory of algebraic stacks, see [30]. We work with algebraic stacks in the fppf topology. Concerning stability of quiver representations we use the sign convention opposite to [20]. For $d, d^{\prime} \in \mathbb{Q}^{n}$ we write $d^{\prime} \leqslant d$ if and only if $d_{i}^{\prime} \leqslant d_{i}$ for all $i$, and we write $d^{\prime}<d$ if $d^{\prime} \leqslant d$ and $d^{\prime} \neq d$.

\section{Moduli Spaces of Quiver Representations}

1.1. Moduli of QUiver RePresentations over arbitrary schemes. Let $Q$ be a finite connected quiver with set of vertices $Q_{0}$ and set of arrows $Q_{1}$. For an arrow $\alpha \in Q_{1}$ we denote $s(\alpha)$ and $t(\alpha)$ the source and target, i.e. $s(\alpha) \stackrel{\alpha}{\longrightarrow} t(\alpha)$.

Definition 1.1. A representation of $Q$ of dimension vector $\left(d_{q}\right)_{q \in Q_{0}}$ over a scheme $Y$ is a tuple $V=\left(\left(V_{q}\right)_{q \in Q_{0}},\left(\varphi_{\alpha}\right)_{\alpha \in Q_{1}}\right)$ where $V_{q}$ is a locally free $\mathcal{O}_{Y}$-module of rank $d_{q}$ and $\varphi_{\alpha}: V_{s(\alpha)} \rightarrow V_{t(\alpha)}$ a homomorphism of $\mathcal{O}_{Y}$-modules. A representation over $Y$ is called free if the underlying $\mathcal{O}_{Y}$-module is free. The notions of homomorphisms of representations, subrepresentations etc. are defined as usual.

DEFINITION 1.2. The representation space of $Q$ for the dimension vector $d=\left(d_{q}\right)_{q \in Q_{0}}$ over the base scheme $S$ is the affine space over $S$

$$
R(Q, d)=\prod_{\alpha \in Q_{1}} \underline{\operatorname{Hom}}_{S}\left(\mathcal{O}_{S}^{\oplus d_{s(\alpha)}}, \mathcal{O}_{S}^{\oplus d_{t(\alpha)}}\right)
$$

where $\underline{\operatorname{Hom}}_{S}\left(\mathcal{O}_{S}^{\oplus d_{s(\alpha)}}, \mathcal{O}_{S}^{\oplus d_{t(\alpha)}}\right)$ is the Hom-scheme that represents the contravariant functor on $S$-schemes $Y \mapsto \operatorname{Hom}_{Y}\left(\mathcal{O}_{Y}^{\oplus d_{s(\alpha)}}, \mathcal{O}_{Y}^{\oplus d_{t(\alpha)}}\right)$. Equivalently, the scheme $R(Q, d)$ can be defined as the scheme that represents the contravariant functor on $S$-schemes

$$
Y \mapsto\{\text { free representations with fixed basis of }(Q, d) \text { over } Y\} .
$$

On the representation space $R(Q, d)$ the group scheme over $S$

$$
G(d)=\prod_{q \in Q_{0}} \mathrm{GL}\left(d_{q}\right)
$$

operates by $\left(g_{q}\right)_{q}\left(\nu_{\alpha}\right)_{\alpha}=\left(g_{t(\alpha)} \nu_{\alpha} g_{s(\alpha)}^{-1}\right)_{\alpha}$ where $\left(g_{q}\right)_{q} \in G(d)(Y),\left(\nu_{\alpha}\right)_{\alpha} \in R(Q, d)(Y)$ for $S$-schemes $Y$. The diagonal subgroup $\mathbb{G}_{m} \cong \Delta \subseteq G(d)$ operates trivially, giving rise to a $\overline{G(d)}=G(d) / \Delta$-operation on $R(Q, d)$.

By $[20]$ the following definition of (semi)stability is equivalent to (semi)stability in the sense of GIT of [24] (with $\prod_{q} \operatorname{det}\left(g_{q}\right)^{-\theta_{q}}$ the character corresponding to $\theta$ ).

DeFINITION 1.3. For a representation $V$ of $Q$ of dimension vector $d=\left(d_{q}\right)_{q \in Q_{0}}$ over an algebraically closed field and a weight $\theta=\left(\theta_{q}\right)_{q \in Q_{0}} \in \mathbb{Q}^{Q_{0}}$, using the notations $\operatorname{dim}(V)=\left(\operatorname{dim}\left(V_{q}\right)\right)_{q \in Q_{0}}$ and $\theta(d)=\sum_{q \in Q_{0}} \theta_{q} d_{q}$, we define:

(a) The representation $V$ is called $\theta$-semistable if $\theta(d)=0$ and every subrepresentation $V^{\prime} \subseteq V$ satisfies $\theta\left(\operatorname{dim}\left(V^{\prime}\right)\right) \leqslant 0$.

(b) A $\theta$-semistable representation $V$ is called $\theta$-stable if the only subrepresentations $V^{\prime} \subseteq V$ with $\theta\left(\operatorname{dim}\left(V^{\prime}\right)\right)=0$ are $V$ and 0 .

DeFINITION 1.4. A representation $V$ of $Q$ of dimension vector $\left(d_{q}\right)_{q \in Q_{0}}$ over a scheme $Y$ is called $\theta$-(semi) stable if for all geometric points $y:$ Spec $k \rightarrow Y$ the representation $V_{y}=V \otimes_{\mathcal{O}_{Y}} k$ is $\theta-($ semi) stable.

The functors on $S$-schemes

$$
Y \mapsto\{\text { free } \theta \text {-(semi)stable representations with fixed basis of }(Q, d) \text { over } Y\}
$$


are represented by open $G(d)$-invariant subschemes $R^{\theta}(Q, d) \subseteq R(Q, d)$ (parametrising $\theta$-semistable representations) and $R^{\theta-s}(Q, d) \subseteq R^{\theta}(Q, d)$ (parametrising $\theta$-stable representations).

In order to construct moduli spaces of representations, following [20] we consider GIT quotients by the (geometrically) reductive group scheme $G(d)$ or $\overline{G(d)}$ (see [1, Section 9] for some results on (geometrically) reductive group schemes). GIT over arbitrary locally noetherian schemes has been worked out first in [29], here we use the formulation of [1] in terms of adequate moduli spaces of Artin stacks.

Let $\mathcal{M}^{\theta}(Q, d)=R(Q, d) /_{\theta} G(d)$ the GIT quotient with respect to $\mathscr{L}=\mathcal{O}_{R(Q, d)}$ with the linearisation determined by the character $\theta$ (if $\theta$ is not integral replace $\theta$ by $l \theta$ for some $l \in \mathbb{Z}_{>0}$ such that $l \theta$ is integral), that is

$$
\mathcal{M}^{\theta}(Q, d)=\operatorname{Proj}_{S} \bigoplus_{k \geqslant 0}\left(p_{*}\left(\mathscr{L}^{\otimes k}\right)\right)^{G(d)}
$$

where $p: R(Q, d) \rightarrow S$ is the structure morphism. We have the quotient morphism

$$
R^{\theta}(Q, d) \rightarrow \mathcal{X}^{\theta}(Q, d) \stackrel{q}{\rightarrow} \mathcal{M}^{\theta}(Q, d) .
$$

where $\mathcal{X}^{\theta}(Q, d)=\left[R^{\theta}(Q, d) / \overline{G(d)}\right]$ is the quotient stack. The next theorem follows from the result in the affine case [1, Thm. 9.1.4] as this property is local [1, Prop. 5.2.9].

TheOREM $1.5([1]) . q: \mathcal{X}^{\theta}(Q, d) \rightarrow \mathcal{M}^{\theta}(Q, d)$ is an adequate moduli space.

In particular, we have the following properties (see [1], cf. also [29]):

(1) The homomorphism $\mathcal{O}_{\mathcal{M}^{\theta}(Q, d)} \rightarrow q_{*} \mathcal{O}_{\mathcal{X}^{\theta}(Q, d)}$ is an isomorphism.

(2) $q$ is surjective, universally closed and universally submersive.

(3) For an algebraically closed field $k$ the morphism $q$ induces a bijection

$$
\left[\mathcal{X}^{\theta}(Q, d)(k)\right] / \sim \rightarrow \mathcal{M}^{\theta}(Q, d)(k)
$$

where $\sim$ is the equivalence relation on the set of isomorphism classes $\left[\mathcal{X}^{\theta}(Q, d)(k)\right]$ defined by $x_{1} \sim x_{2}$ if $\overline{\left\{x_{1}\right\}} \cap \overline{\left\{x_{2}\right\}} \neq \varnothing$ in $\mathcal{X}^{\theta}(Q, d)_{s}$ for $x_{1}, x_{2} \in \mathcal{X}^{\theta}(Q, d)(k)$ over $s:$ Spec $k \rightarrow S$.

(4) Assume that $S$ is locally noetherian. Then $\mathcal{M}^{\theta}(Q, d)$ is of finite type over $S$ and $q_{*} \mathscr{F}$ is coherent for every coherent $\mathcal{O}_{\mathcal{X}^{\theta}(Q, d)}$-module $\mathscr{F}$.

(5) $q$ is universal for morphisms to schemes, i.e. for an $S$-scheme $Z$ the map

$$
\operatorname{Mor}_{S}\left(\mathcal{M}^{\theta}(Q, d), Z\right) \rightarrow \operatorname{Mor}_{S}\left(\mathcal{X}^{\theta}(Q, d), Z\right), \quad f \mapsto f \circ q
$$

is a bijection.

(6) Let $Y \rightarrow \mathcal{M}^{\theta}(Q, d)$ be a flat morphism of $S$-schemes. Then $\mathcal{X}^{\theta}(Q, d) \times \mathcal{M}^{\theta}(Q, d)$ $Y \rightarrow Y$ is an adequate moduli space.

Statement (5) means that $R^{\theta}(Q, d) \rightarrow \mathcal{M}^{\theta}(Q, d)$ is a categorical quotient. Restricting to the open subscheme of stable points $R^{\theta-s}(Q, d) \subseteq R^{\theta}(Q, d)$ there is the following result corresponding to the notion of geometric quotient.

Corollary 1.6. Let $\mathcal{X}^{\theta-s}(Q, d)=\left[R^{\theta-s}(Q, d) / \overline{G(d)}\right]$ and $\mathcal{M}^{\theta-s}(Q, d)$ the image of $\mathcal{X}^{\theta-s}(Q, d)$ in $\mathcal{M}^{\theta}(Q, d)$. Then $\mathcal{X}^{\theta-s}(Q, d) \rightarrow \mathcal{M}^{\theta-s}(Q, d)$ is an adequate moduli space. For algebraically closed fields $k$ the map $\left[\mathcal{X}^{\theta-s}(Q, d)(k)\right] \rightarrow \mathcal{M}^{\theta-s}(Q, d)(k)$ is a bijection, where $\left[\mathcal{X}^{\theta-s}(Q, d)(k)\right]$ denotes the set of isomorphism classes of objects in $\mathcal{X}^{\theta-s}(Q, d)(k)$.

In addition to these standard GIT results, in our situation we have:

Proposition 1.7. The operation of $\overline{G(d)}$ on $R^{\theta-s}(Q, d)$ is free and the morphism $R^{\theta-s}(Q, d) \rightarrow \mathcal{M}^{\theta-s}(Q, d)$ is a $\overline{G(d)}$-torsor in the fppf topology. 
Proof. We first show that the operation of $\overline{G(d)}$ has trivial stabilisers. For an $S$-scheme $Y$ the stabiliser $\operatorname{St}(v) \subseteq G(d)_{Y}$ of an element $v \in R^{\theta-s}(Q, d)(Y)$ contains the diagonal group scheme $\Delta_{Y} \subseteq G(d)_{Y}$ and coincides with the automorphism group scheme $\operatorname{Aut}_{Y}(V)$ of the corresponding free representation $V=\left(\left(V_{q}\right)_{q},\left(\nu_{\alpha}\right)_{\alpha}\right)$ of $(Q, d)$ over $Y$. The automorphism group scheme $\operatorname{Aut}_{Y}(V)$ is open dense in the endomorphism scheme $\operatorname{End}_{Y}(V)$. As the $\mathcal{O}_{Y}$-module $\mathscr{E} n d_{Y}(V)$ is the kernel of the homomorphism of locally free sheaves $\bigoplus_{q} \mathscr{E} n d_{Y}\left(V_{q}\right) \rightarrow \bigoplus_{\alpha} \mathscr{H} o m_{Y}\left(V_{s(\alpha)}, V_{t(\alpha)}\right),\left(\varphi_{q}\right)_{q} \mapsto\left(\nu_{\alpha} \varphi_{s(\alpha)}-\right.$ $\left.\varphi_{t(\alpha)} \nu_{\alpha}\right)_{\alpha}$, we have $\underline{\operatorname{End}}_{Y}(V)=\mathbb{A}_{Y}(\mathscr{C})$ with $\mathscr{C}=\operatorname{coker}\left(\bigoplus_{\alpha} \mathscr{H} o m_{Y}\left(V_{s(\alpha)}, V_{t(\alpha)}\right)^{\vee} \rightarrow\right.$ $\left.\bigoplus_{q} \mathscr{E} n d_{Y}\left(V_{q}\right)^{\vee}\right)$. Since $v$ is stable, all geometric fibres of $\operatorname{St}(v)=\underline{\text { Aut }}_{Y}(V)$ are of dimension 1 (containing the diagonal group $\Delta$ ), therefore all geometric fibres of $\underline{\text { End }}_{Y}(V)$ are 1-dimensional affine spaces, so $\underline{\operatorname{End}}_{Y}(V)$ is an affine line bundle over $Y$. It follows that $\operatorname{St}(v)=\underline{\operatorname{Aut}}_{Y}(V)=\Delta_{Y}$.

We show that the operation is proper, that is $\Psi: \overline{G(d)} \times R^{\theta-s}(Q, d) \rightarrow R^{\theta-s}(Q, d) \times$ $R^{\theta-s}(Q, d),(g, v) \mapsto(g v, v)$ is a proper morphism, using the valuative criterion [12, II, (7.3.8)]. We can assume we have the base scheme $S=\operatorname{Spec} \mathbb{Z}$. Let $A$ be a discrete valuation ring with maximal ideal $\mathfrak{m}$, field of fractions $K$ and $k=A / \mathfrak{m}$, and let $t$ be a generator of $\mathfrak{m}$. Given a morphism Spec $A \rightarrow R^{\theta-s}(Q, d) \times R^{\theta-s}(Q, d)$ we have to show that the map $\operatorname{Mor}_{R^{\theta-s}(Q, d) \times R^{\theta-s}(Q, d)}\left(\operatorname{Spec} A, \overline{G(d)} \times R^{\theta-s}(Q, d)\right) \rightarrow$ $\operatorname{Mor}_{R^{\theta-s}(Q, d) \times R^{\theta-s}(Q, d)}\left(\operatorname{Spec} K, \overline{G(d)} \times R^{\theta-s}(Q, d)\right)$ coming from the canonical homomorphism $A \rightarrow K$ is surjective (it is injective because the morphism is separated). The morphism Spec $A \rightarrow R^{\theta-s}(Q, d) \times R^{\theta-s}(Q, d)$ corresponds to a pair of representations $V^{\prime}=\left(\left(V_{q}^{\prime}\right)_{q},\left(\nu_{\alpha}^{\prime}\right)_{\alpha}\right), V=\left(\left(V_{q}\right)_{q},\left(\nu_{\alpha}\right)_{\alpha}\right)$ of $(Q, d)$ over Spec $A$ and a morphism Spec $K \rightarrow \overline{G(d)} \times R^{\theta-s}(Q, d)$ making the diagram arising from the above maps (the usual diagram when applying the valuative criterion) commutative corresponds to a pair $\left(g, V^{\prime \prime}\right)$ such that $V^{\prime \prime}=V_{K}, g V_{K}=V_{K}^{\prime}$. The element $g \in \overline{G(d)}(K)$ corresponds to a family of matrices with coefficients in $K$ up to a common factor in $K^{*}$, so we can represent it by a family of matrices $\left(M_{q}\right)_{q}$ with coefficients in $A$ and choose bases of $V_{q}$ and $V_{q}^{\prime}$ such that all $M_{q}$ are diagonal with diagonal entries of the form $t^{e}$. Let $l$ be the minimal integer such that $t^{l}$ occurs as a diagonal entry of some $M_{q}$. Considering the matrices for the homomorphisms $\nu_{\alpha}, \nu_{\alpha}^{\prime}$ with respect to the chosen bases, the $(i, j)$-entries for the two matrices for a given $\alpha$ differ by a factor $t^{e} \neq 1$ if the diagonal entries for the corresponding pairs of basis elements differ by such a factor. In this case modulo $\mathfrak{m}$ one of the two $(i, j)$-entries vanishes. Let $W_{q}^{\prime} \subseteq V_{q}^{\prime}$ be the submodules generated by those basis elements which correspond to the diagonal entries of the form $t^{l}$, and similary $\left(U_{q}\right)_{q}$ the submodules of $\left(V_{q}\right)_{q}$ generated by the basis elements corresponding to the remaining diagonal entries. Then central fibres of the modules $W_{q}^{\prime} \subseteq V_{q}^{\prime}$ form a subrepresentation $W_{k}^{\prime} \subseteq V_{k}^{\prime}$ and the central fibres of the modules $U_{q} \subseteq V_{q}$ form a subrepresentation $U_{k} \subset V_{k}$. If these were proper nonzero subrepresentations, then, because $V_{k}, V_{k}^{\prime}$ are $\theta$-stable, both subrepresentations would satisfy $\theta\left(W_{k}^{\prime}\right)<0, \theta\left(U_{k}\right)<0$, which is impossible. Therefore $W_{k}^{\prime}=V_{k}^{\prime}, U_{k}=0$. It follows that the element $g \in \overline{G(d)}(K)$ can be represented by matrices with coefficients in $A \backslash \mathfrak{m}$. These matrices are invertible as matrices over $A$ and form the required element in $\overline{G(d)}(A)$.

Being proper and quasifinite, the morphism $\Psi: \overline{G(d)} \times R^{\theta-s}(Q, d) \rightarrow R^{\theta-s}(Q, d) \times$ $R^{\theta-s}(Q, d)$ is finite by [12, III (1), (4.4.2)] or [12, IV (3), (8.11.1)]. The morphism $\Psi$ is a monomorphism because the operation has trivial stabilisers. As finite epimorphisms of rings are surjective (see [22, Ch. IV, Prop. 1.7]), it follows that $\Psi$ is a closed embedding, i.e. the operation $\overline{G(d)} \times R^{\theta-s}(Q, d) \rightarrow R^{\theta-s}(Q, d)$ is free. 
The sheaf-quotient (fppf) $\left(R^{\theta-s}(Q, d) / \overline{G(d)}\right)_{\text {fppf }}$, i.e. the quotient in the category of fppf-sheaves, is the sheaf associated to the presheaf $Y \mapsto R^{\theta-s}(Q, d)(Y) / \overline{G(d)}(Y)$ on the fppf-site of $S$-schemes (see e.g. [4, 3.4.1]). As the operation $\overline{G(d)} \times R^{\theta-s}(Q, d) \rightarrow$ $R^{\theta-s}(Q, d)$ is free and a geometric quotient $\pi: R^{\theta-s}(Q, d) \rightarrow R^{\theta-s}(Q, d) / \overline{G(d)}$ exists, by $\left[2\right.$, Thm. 6] the canonical morphism $\left(R^{\theta-s}(Q, d) / \overline{G(d)}\right)_{\mathrm{fppf}} \rightarrow R^{\theta-s}(Q, d) / \overline{G(d)}$ is an isomorphism and the morphism $\pi$ is fppf. Since the sheaf-quotient $\left(R^{\theta-s}(Q, d) / \overline{G(d)}\right)_{\text {fppf }}$ has the property that $\overline{G(d)} \times R^{\theta-s}(Q, d) \rightarrow R^{\theta-s}(Q, d) \times{ }_{\left(R^{\theta-s}(Q, d) / \overline{G(d)}\right)_{\mathrm{fppf}}} R^{\theta-s}(Q, d)$ is an isomorphism (see e.g. $[4,3.4 .5]$ ), it follows that the morphism of schemes $R^{\theta-s}(Q, d) \rightarrow R^{\theta-s}(Q, d) / \overline{G(d)}$ is a $\overline{G(d)}$-torsor in the fppf topology.

The tautological $G(d)$-equivariant representation $U_{R^{\theta-s}(Q, d)}$ on $R^{\theta-s}(Q, d)$ can be made into a $\overline{G(d)}$-equivariant representation if and only if the dimension vector $d$ is indivisible, i.e. the set of integers $d_{q}$ is coprime, see [20, Proof of Prop. 5.3]. In this case by descent along the $\overline{G(d)}$-torsor $R^{\theta-s}(Q, d) \rightarrow \mathcal{M}^{\theta-s}(Q, d)$ one obtains a representation $U_{\mathcal{M}^{\theta-s}(Q, d)}$ on $\mathcal{M}^{\theta-s}(Q, d)$.

THEOREM 1.8. For an indivisible dimension vector $d=\left(d_{q}\right)_{q \in Q_{0}}$ the scheme $\mathcal{M}^{\theta-s}(Q, d)$ with universal representation $U_{\mathcal{M}^{\theta-s}(Q, d)}$ is a fine moduli space of $\theta$ stable representations of $(Q, d)$ in the sense that it represents the contravariant functor on $S$-schemes

$$
Y \mapsto\{\theta \text {-stable representations of }(Q, d) \text { over } Y\} / \sim
$$

of $\theta$-stable representations up to isomorphism over a Zariski cover.

Proof. We denote the above functor $F^{\theta-s}(Q, d)$ and $q: R^{\theta-s}(Q, d) \rightarrow \mathcal{M}^{\theta-s}(Q, d)$ the quotient morphism. We have the morphisms $\mathcal{M}^{\theta-s}(Q, d) \rightarrow F^{\theta-s}(Q, d),(f: Y \rightarrow$ $\left.\mathcal{M}^{\theta-s}(Q, d)\right) \mapsto f^{*} U_{\mathcal{M}^{\theta-s}(Q, d)}$ and $F^{\theta-s}(Q, d) \rightarrow \mathcal{M}^{\theta-s}(Q, d), V \mapsto f_{V}$, where $f_{V}$ is locally defined as $f_{V}=q \circ \tilde{f}_{V}$ for free $V$ and $\tilde{f}_{V}: Y \rightarrow R^{\theta-s}(Q, d)$ is determined by $V$ and a choice of basis of $V$, using that $R^{\theta-s}(Q, d)$ with $U_{R^{\theta-s}(Q, d)}$ represents the functor of $\theta$-stable free representations with fixed bases.

To show that the composition $F^{\theta-s}(Q, d) \rightarrow \mathcal{M}^{\theta-s}(Q, d) \rightarrow F^{\theta-s}(Q, d)$ is the identity, it is enough to show that $f_{V}^{*} U_{\mathcal{M}^{\theta-s}(Q, d)} \cong V$ for free representations $V$. It is $f_{V}^{*} U_{\mathcal{M}^{\theta-s}(Q, d)}=\left(q \circ \tilde{f}_{V}\right)^{*} U_{\mathcal{M}^{\theta-s}(Q, d)} \cong \tilde{f}_{V}^{*} U_{R^{\theta-s}(Q, d)} \cong V$.

To show that the composition $\mathcal{M}^{\theta-s}(Q, d) \rightarrow F^{\theta-s}(Q, d) \rightarrow \mathcal{M}^{\theta-s}(Q, d)$ is the identity, it suffices to show that $f=f_{f^{*} U_{\mathcal{M}^{\theta-s}(Q, d)}}$ for $f: Y \rightarrow \mathcal{M}^{\theta-s}(Q, d)$ such that $f^{*} U_{\mathcal{M}^{\theta-s}(Q, d)}$ is free. Let $p_{Y}: Y^{\prime} \rightarrow Y$ be an fppf morphism such that the $\overline{G(d)}$-torsor $R_{Y}=Y_{f \times} \times{ }_{q} R^{\theta-s}(Q, d) \rightarrow Y$ becomes a trivial torsor $R_{Y^{\prime}} \rightarrow Y^{\prime}$ after base extension by $p_{Y}$. A section $Y^{\prime} \rightarrow R_{Y^{\prime}}$ gives a morphism $\tilde{f}^{\prime}: Y^{\prime} \rightarrow R^{\theta-s}(Q, d)$ which satisfies $q \circ \tilde{f}^{\prime}=$ $f \circ p_{Y}$. We have $\left(\tilde{f}^{\prime}\right)^{*} U_{R^{\theta-s}(Q, d)} \cong p_{Y}^{*} f^{*} U_{\mathcal{M}^{\theta-s}(Q, d)} \cong p_{Y}^{*}\left(\tilde{f}_{f^{*} U_{\mathcal{M}^{\theta-s}(Q, d)}}\right)^{*} U_{R^{\theta-s}(Q, d)}$. Therefore $\tilde{f}^{\prime}$ coincides with $\tilde{f}_{f^{*} U_{\mathcal{M}^{\theta-s}(Q, d)}} \circ p_{Y}$ up to an element of $\overline{G(d)}$ that arises from comparison of the two bases coming from $U_{R^{\theta-s}(Q, d)}$ of these isomorphic representations. Composing with $q$, it follows $f \circ p_{Y}=f_{f^{*} U_{\mathcal{M}^{\theta-s}(Q, d)}} \circ p_{Y}$, and thus $f=f_{f^{*} U_{\mathcal{M}^{\theta-s}(Q, d)}}$ since $p_{Y}$ is an epimorphism.

The equivalence relation on isomorphism classes of geometric points of $\mathcal{X}^{\theta}(Q, d)$ in (3) after theorem 1.5, or equivalently expressed as GIT-equivalence on $R^{\theta}(Q, d)(k)$ defined by $x_{1} \sim x_{2}$ if $\overline{G(d)_{s} x_{1}} \cap \overline{G(d)_{s} x_{2}} \neq \varnothing$ in $R^{\theta}(Q, d)_{s}$ for $x_{1}, x_{2} \in R^{\theta}(Q, d)(k)$ over $s:$ Spec $k \rightarrow S$, is by [20] equivalent to S-equivalence of semistable representations as in the following definition. 
Definition 1.9. Two $\theta$-semistable representations $V, V^{\prime}$ of $Q$ over an algebraically closed field are called $S$-equivalent if there are filtrations in the category of $\theta$-semistable representations $0=V_{0} \subsetneq \ldots \subsetneq V_{l}=V$ and $0=V_{0}^{\prime} \subsetneq \ldots \subsetneq V_{l}^{\prime}=V^{\prime}$ with $\theta$-stable quotients such that $\bigoplus_{i} V_{i+1} / V_{i} \cong \bigoplus_{i} V_{i+1}^{\prime} / V_{i}^{\prime}$.

In particular, $\theta$-stable representations are $\mathrm{S}$-equivalent if and only if they are isomorphic. Note that the $\mathrm{S}$ in S-equivalence doesn't refer to the base scheme $S$.

COROLlary 1.10. Via the morphism $R^{\theta}(Q, d) \rightarrow \mathcal{M}^{\theta}(Q, d)$ any $\theta$-semistable representation of $(Q, d)$ over an $S$-scheme $Y$ determines a morphism $Y \rightarrow \mathcal{M}^{\theta}(Q, d)$ over $S$. This way for a geometric point $s:$ Spec $k \rightarrow S$ the geometric points $\mathcal{M}^{\theta}(Q, d)_{s}(k)$ over $s$ are in bijection with the $S$-equivalence classes of $\theta$-semistable representations of $(Q, d)$ over $k$.

1.2. DeCOMPOSITION OF THE WEIGHT SPACE AND Limits OF MODULI SPACES OF QUIVER REPRESENTATIONS. In the case of GIT quotients of projective varieties $X$ over an algebraically closed field the chamber structure of the space $\mathrm{NS}^{G}(X)_{\mathbb{Q}}$, where $\mathrm{NS}^{G}(X)$ is the Néron-Severi group of $G$-equivariant line bundles, has been described in $[31,7,25]$, and in the case $X=R(Q, d)$ of quivers (without oriented cycles) in $[16,5]$.

Let $Q$ be a finite connected quiver, $d=\left(d_{q}\right)_{q \in Q_{0}}$ a dimension vector, $S$ be a scheme and $R(Q, d)$ the representation space over $S$. We have the space $\mathbb{Q}^{Q_{0}}$ of fractional linearisations of the line bundle $\mathcal{O}_{R(Q, d)}$.

DEFINITION 1.11. We define the weight space

$$
H(Q, d)=\left\{\theta \in \mathbb{Q}^{Q_{0}} \mid \theta(d)=0\right\}
$$

and

$$
C(Q, d)=\left\{\theta \in H(Q, d) \mid R^{\theta}(Q, d) \neq \varnothing\right\} .
$$

For the finitely many dimension vectors $0<d^{\prime}<d$ we define

$$
\begin{aligned}
H_{d^{\prime}} & =\left\{\theta \in H(Q, d) \mid \theta\left(d^{\prime}\right)=0\right\}, \\
H_{d^{\prime}}^{<0} & =\left\{\theta \in H(Q, d) \mid \theta\left(d^{\prime}\right)<0\right\}, \\
H_{d^{\prime}}^{\leqslant 0} & =\left\{\theta \in H(Q, d) \mid \theta\left(d^{\prime}\right) \leqslant 0\right\} .
\end{aligned}
$$

REMARK 1.12. Let the base scheme be $S=\operatorname{Spec} k, k$ an algebraically closed field.

(1) Existence of subrepresentations of a given dimension vector is a closed condition on $R(Q, d)$, see [26, Lemma 3.1]. We call a dimension vector $0<d^{\prime}<d$ a generic dimension vector if there is a non-empty open subscheme of $R(Q, d)$ of representations that have a subrepresentation of dimension vector $d^{\prime}$, or equivalently, all representations of dimension vector $d$ have a subrepresentation of dimension vector $d^{\prime}$. There is a dense open subset in $R(Q, d)$ of representations which only have subrepresentations of generic dimension vectors.

(2) The set of weights that allow (semi)stable representations is determined by the generic dimension vectors: the intersection $\bigcap_{d^{\prime} \text { generic }} H_{d^{\prime}}^{<0}$ is the set of weights $\theta \in H(Q, d)$ such that $\theta$-stable representations exist, and

$$
C(Q, d)=\bigcap_{d^{\prime} \text { generic }} H_{d^{\prime}}^{\leq 0}
$$

Thus $C(Q, d)$ is a convex polyhedral cone in $H(Q, d)$, and it is full-dimensional if there is a $\theta \in H(Q, d)$ such that $\theta$-stable representations exist. If the quiver $Q$ has no oriented cycles then $C(Q, d)$ is strongly convex.

(3) By [6, Cor. 1], improving the results of [26] (see Thm. 3.3, Thm. 5.4 and the text following the proof of Thm. 5.4 in [26]), the generic dimension vectors, and thus the cone $C(Q, d)$, are combinatorially determined. 
Definition 1.13. Weights $\theta, \theta^{\prime} \in H(Q, d)$ are called GIT equivalent if

$$
R^{\theta}(Q, d)=R^{\theta^{\prime}}(Q, d)
$$

We denote the GIT equivalence class of $\theta$ by

$$
C_{\theta}=\left\{\theta^{\prime} \in H(Q, d) \mid R^{\theta}(Q, d)=R^{\theta^{\prime}}(Q, d)\right\}
$$

and denote

$$
\bar{C}_{\theta}=\left\{\theta^{\prime} \in H(Q, d) \mid R^{\theta}(Q, d) \subseteq R^{\theta^{\prime}}(Q, d)\right\} .
$$

For the standard results in the following proposition see [5] (following [25]; proven for quivers without oriented cycles, but the results and methods of proof also apply to quivers with oriented cycles).

Proposition 1.14. Let $S=$ Spec $k, k$ an algebraically closed field. For $\theta \in C(Q, d)$ the set $\bar{C}_{\theta}$ is a convex polyhedral cone and relint $\left(\bar{C}_{\theta}\right)=C_{\theta}$. The cones $\bar{C}_{\theta}$ for $\theta \in C(Q, d)$ form a finite fan with support $C(Q, d)$.

In the situation of proposition 1.14 the decomposition of $C(Q, d)$ into GIT equivalence classes is determined by segments of the hyperplanes $H_{d^{\prime}}$. The relevant part $W_{d^{\prime}} \subseteq H_{d^{\prime}}$ consists of those $\theta$ such that there is a $\theta$-semistable representation $V$ with a subrepresentation $V^{\prime} \subset V$ of dimension vector $d^{\prime}$ such that $\theta\left(V^{\prime}\right)=0$. Since in this case both $V^{\prime}$ and $V / V^{\prime}$ are also $\theta$-semistable, we have the equivalent condition that there is a $\theta$-semistable $V$ of the form $V \cong V^{\prime} \oplus V^{\prime \prime}$ with $V^{\prime}, V^{\prime \prime} \theta$-semistable of dimension vector $d^{\prime}, d-d^{\prime}$, thus:

$$
W_{d^{\prime}}=C\left(Q, d^{\prime}\right) \cap C\left(Q, d-d^{\prime}\right)
$$

Using the fact from remark 1.12.(3) that the sets $C(Q, d)$ for any $d$ are combinatorially determined, this implies the following result.

Proposition 1.15. The set $C(Q, d) \subseteq H(Q, d)$ and its decomposition into GIT equivalence classes over an algebraically closed field $k$ do not depend on the base field $k$.

It follows that the above results concerning the decomposition of $H(Q, d)$, in particular proposition 1.14 , also apply to the case of an arbitrary base scheme $S$. The weight space decomposition over $S$ can be determined by looking at an arbitrary fibre (or the situation over any algebraically closed field).

Assume that stable representations for some weight $\theta$ exist. Then $C(Q, d)$ is fulldimensional. The sets $W_{d^{\prime}}$ of codimension one are called walls. Walls whose intersections with $\operatorname{int}(C(Q, d))$ are nonempty are called inner walls, otherwise outer walls. A weight $\theta \in H(Q, d)$ is said to be generic if $R^{\theta}(Q, d)=R^{\theta-s}(Q, d) \neq \varnothing$. The GIT equivalence classes of generic weights are open sets called chambers, the connected components of weights $\theta \in \operatorname{int} C(Q, d)$ not contained in an inner wall.

By variation of geometric invariant theory quotients (VGIT), cf. [31], [7], quiver varieties of nearby weights are related. Let $C_{\theta_{0}}, C_{\theta} \subset C(Q, d)$ be GIT equivalence classes such that $C_{\theta_{0}} \subseteq \bar{C}_{\theta}$. Then the inclusion $R^{\theta}(Q, d) \subseteq R^{\theta_{0}}(Q, d)$ induces a morphism over $S$

$$
\varphi_{\theta, \theta_{0}}: \mathcal{M}^{\theta}(Q, d) \rightarrow \mathcal{M}^{\theta_{0}}(Q, d)
$$

The morphisms $\varphi_{\theta, \theta_{0}}: \mathcal{M}^{\theta}(Q, d) \rightarrow \mathcal{M}^{\theta_{0}}(Q, d)$ for representatives of the GIT equivalence classes in $C(Q, d)$ form an inverse system and we can consider its inverse limit $\lim _{\theta} \mathcal{M}^{\theta}(Q, d)$.

Proposition 1.16. Let $Q$ be a quiver and $\left(d_{q}\right)_{q \in Q_{0}}$ be an indivisible dimension vector. Assume that stable representations for some weight $\theta$ exist. Then the functor 
$\varliminf_{\theta} \mathcal{M}^{\theta}(Q, d)$ is isomorphic to the closed subfunctor of the product of $\mathcal{M}^{\theta}(Q, d)$ for generic $\theta$

(1) $Y \mapsto\left\{\begin{array}{l|l}\left(\varphi_{\theta}\right)_{\theta} \in \prod_{\theta \text { generic }} \mathcal{M}^{\theta}(Q, d)(Y) & \begin{array}{l}\forall \theta, \theta^{\prime} \text { generic } \forall \tau \in \bar{C}_{\theta} \cap \bar{C}_{\theta^{\prime}}: \\ \varphi_{\theta, \tau} \circ \varphi_{\theta}=\varphi_{\theta^{\prime}, \tau} \circ \varphi_{\theta^{\prime}}\end{array}\end{array}\right\}$

Proof. The limit functor is isomorphic to this functor since each $\tau \in C(Q, d)$ is contained in some $\bar{C}_{\theta}$ for a generic $\theta$. This functor is a closed subfunctor of the product because $\mathcal{M}^{\tau}(Q, d)$ is a scheme separated over $S$ (use $[13,(5.2 .5)]$ ).

\section{Weight SPACE DeCOMPosition AND MOduli SPACES FOR SOME BIPARTITE QUIVERS}

2.1. The Quivers $P_{n}, Q_{n}$ And the structure of the Weight Space. We define the quivers $P_{n}$ and $Q_{n}$ with fixed dimension vectors (cf. introduction).

DeFinition 2.1.

(a) Let $Q_{n}=\left(\left\{p, q_{1}, \ldots, q_{n}\right\},\left\{\alpha_{1}, \ldots, \alpha_{n}\right\}\right)$ where $s\left(\alpha_{i}\right)=q_{i}, t\left(\alpha_{i}\right)=p$ with dimension vector given by $d_{p}=2$ and $d_{q_{i}}=1$.

(b) Let $P_{n}=\left(\left\{p_{1}, p_{2}, q_{1}, \ldots, q_{n}\right\},\left\{\alpha_{1,1}, \alpha_{1,2}, \ldots, \alpha_{n, 1}, \alpha_{n, 2}\right\}\right)$ where $s\left(\alpha_{i, j}\right)=q_{i}$, $t\left(\alpha_{i, j}\right)=p_{j}$ with dimension vector $(1, \ldots, 1)$.

For these quivers with dimension vectors we determine the decomposition of the weight space as considered in subsection 1.2. In the following when studying the structure of GIT classes in the cone $C(Q) \subset H(Q)$ for $Q=Q_{n}, P_{n}$ we restrict to the intersection with a certain affine hyperplane. In the case $Q_{n}$ this yields the hypersimplex $\Delta(2, n)$, in the case $P_{n}$ the product of simplices $\Delta^{1} \times \Delta^{n-1}$.

REMARK 2.2. Decomposition of the weight space for $Q_{n}$.

(1) A weight for the quiver $Q_{n}$ is given by a tuple $\theta=\left(\eta, \theta_{1}, \ldots, \theta_{n}\right)$ where $\theta_{p}=\eta$, $\theta_{q_{i}}=\theta_{i}$. The weight space is the $n$-dimensional hyperplane $H\left(Q_{n}\right)=\{\theta \in$ $\left.\mathbb{Q}^{n+1} \mid 2 \eta=-\sum_{i} \theta_{i}\right\} \subset \mathbb{Q}^{n+1}$. Omitting the coordinate $\eta$ we have an isomorphism $H\left(Q_{n}\right) \cong \mathbb{Q}^{n}$, with basis $e_{1}, \ldots, e_{n} \in \mathbb{Q}^{n}$ and coordinates $\theta_{1}, \ldots, \theta_{n}$. A semistable representation exists if and only if $0 \leqslant \theta_{i} \leqslant \frac{1}{2} \sum_{i=1}^{n} \theta_{i}$ for all $i$, this defines a full-dimensional strongly convex polyhedral cone $C\left(Q_{n}\right) \subset H\left(Q_{n}\right)$. The affine hyperplane $\left\{\sum_{i=1}^{n} \theta_{i}=2\right\}$ intersects this cone in the hypersimplex

$$
C\left(Q_{n}\right) \cap\left\{\sum_{i=1}^{n} \theta_{i}=2\right\}=\operatorname{conv}\left\{e_{i}+e_{j} \mid i \neq j\right\}=\Delta(2, n) .
$$

(2) For $\theta=\left(\theta_{1}, \ldots, \theta_{n}\right) \in \Delta(2, n)$ a $\theta$-semistable representation that is not $\theta$ stable exists if and only if $\theta$ is contained in one of the following walls. The outer walls are of the form $W_{i}=\left\{\theta_{i}=0\right\} \cap \Delta(2, n)=\operatorname{conv}\left\{e_{j}+e_{k} \mid j, k \neq i\right\} \cong$ $\Delta(2, n-1)$ and $W_{\{\{i\},\{1, \ldots, n\} \backslash\{i\}\}}=\left\{\theta_{i}=1\right\} \cap \Delta(2, n)=\operatorname{conv}\left\{e_{i}+e_{j} \mid j \in\right.$ $\{1 \ldots, n\} \backslash\{i\}\} \cong \Delta(1, n-1)=\Delta^{n-2}$. The inner walls are

$$
\begin{gathered}
W_{\left\{J, J^{\complement}\right\}}=\left\{\theta \in \Delta(2, n) \mid \sum_{i \in J} \theta_{i}=1\right\}=\operatorname{conv}\left\{e_{i}+e_{j} \mid i \in J, j \in J^{\complement}\right\} \\
\text { for } J \subset\{1, \ldots, n\}, 2 \leqslant|J| \leqslant n-2, J^{\complement}=\{1, \ldots, n\} \backslash J . \text { It is } \\
W_{\left\{J, J^{\complement}\right\}} \cong \Delta^{|J|-1} \times \Delta^{n-|J|-1} .
\end{gathered}
$$

(3) An inner wall $W_{\left\{J, J^{\complement}\right\}}$ intersects the boundary of $\Delta(2, n)$ as follows: $W_{\left\{J, J^{\complement}\right\}} \cap$

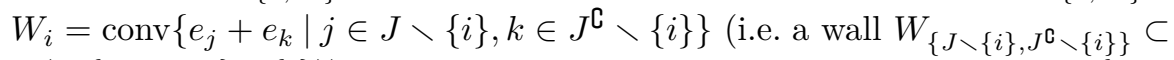
$\Delta(2,\{1, \ldots, n\} \backslash\{i\}))$ and, assuming $i \in J, W_{\left\{J, J^{\mathrm{C}}\right\}} \cap W_{\left\{\{i\},\{i\}^{\mathrm{C}}\right\}}=\operatorname{conv}\left\{e_{i}+\right.$ $\left.e_{j} \mid j \in J^{\complement}\right\}=\Delta^{\left|J^{\complement}\right|-1}$. 
(4) Distinct inner walls $W_{\left\{J_{1}, J_{1}^{\complement}\right\}}, W_{\left\{J_{2}, J_{2}^{\complement}\right\}} \subset \Delta(2, n)$ do not intersect in the interior of $\Delta(2, n)$ if and only if the two partitions of $\{1, \ldots, n\}$ into two subsets are compatible in the sense that there is an inclusion between the sets $J_{1}, J_{1}^{\complement}$ and $J_{2}, J_{2}^{\complement}$. In this case, if we assume $J_{1} \subset J_{2}$, the intersection $W_{\left\{J_{1}, J_{1}^{\mathrm{C}}\right\}} \cap W_{\left\{J_{2}, J_{2}^{\mathrm{C}}\right\}}=\operatorname{conv}\left\{e_{j}+e_{k} \mid j \in J_{1}, k \in J_{2}^{\complement}\right\}=\Delta^{\left|J_{1}\right|-1} \times \Delta^{\left|J_{2}^{\mathrm{C}}\right|-1}$ is a wall of the face $\Delta\left(2, J_{1} \cup J_{2}^{\complement}\right)$ of $\Delta(2, n)$.

We have the representation space $R\left(Q_{n}\right)=\left(\mathbb{A}^{2}\right)^{n}$ with the operation of GL(2) $\times$ $\left(\mathbb{G}_{m}\right)^{n}$. A free representation of $Q_{n}$ over a scheme $Y$ after a choice of a basis can be expressed as a tuple $V=\left(s_{1}, \ldots, s_{n}\right)$ of $n$ sections of $\mathbb{A}_{Y}^{2} \rightarrow Y$. We write $s_{i} \sim s_{j}$ if $s_{i}$ and $s_{j}$ are in the same $\mathbb{G}_{m}$-orbit.

LEMmA 2.3. Let $V=\left(s_{1}, \ldots, s_{n}\right)$ be a representation of $Q_{n}$ over an algebraically closed field that is semistable with respect to some weight in the interior of $\Delta(2, n)$, in particular all $s_{i}$ are nonzero. Let the partition $\{1, \ldots, n\}=\bigsqcup_{l} J_{l}$ be defined by $i, j \in J_{l}$ for some $l$ if and only if $s_{i} \sim s_{j}$. The set $\Theta(V)=\{\theta \in \Delta(2, n) \mid V$ is $\theta$-semistable $\}$ is a closed convex polytope in $\Delta(2, n)$ with the following properties:

(a) We have $\Theta(V)=\bigcap_{l}\left\{\theta \in \Delta(2, n) \mid \sum_{i \in J_{l}} \theta_{i} \leqslant 1\right\}$, i.e. $\Theta(V)$ is bounded within $\Delta(2, n)$ by the inner walls $W_{\left\{J_{l}, J_{l}^{\complement}\right\}}$ for $2 \leqslant\left|J_{l}\right| \leqslant n-2$. Any two distinct of these walls do not intersect in the interior of $\Delta(2, n)$.

(b) The sets of vertices and edges of $\Theta(V)$ are subsets of the sets of vertices and edges of $\Delta(2, n) . \Theta(V)=\operatorname{conv}\left\{e_{i}+e_{j} \mid i \in J_{l}, j \in J_{l^{\prime}}\right.$ for some $\left.l \neq l^{\prime}\right\}$.

(c) $\Theta(V)$ is full dimensional if and only if there are $\geqslant 3$ classes $J_{l}$, or equivalently, $V$ is stable with respect to some weight. Otherwise there are two classes $J$ and $J^{\complement}$ and $\Theta(V)=W_{\left\{J, J^{\complement}\right\}}$.

Proof. For (a) note that the nontrivial subrepresentations of $V$ with one-dimensional subspace in $V_{q}$ are given by the sets $J_{l}$ (the spaces $\left(V_{q_{j}}\right)_{j \in J_{l}}$ together with the onedimensional subspace of $V_{p}$ determined by $\left.\left(s_{j}\right)_{j \in J_{l}}\right)$. That the walls do not intersect in the interior follows from remark 2.2.(4). Concerning (b), as the inner walls bounding $\Theta(V)$ do not intersect in the interior of $\Delta(2, n)$, the first statement follows inductively using remark 2.2.(3) and (4). Then $\Theta(V)$ is the convex hull of the vertices of $\Delta(2, n)$ it contains. The statements in (c) are easy to show.

The property that the sets of vertices and edges of $\Theta(V)$ are subsets of the sets of vertices and edges of $\Delta(2, n)$ means that $\Theta(V)$ is a matroid polytope. Matroid polytopes were defined in [8] (see also [17, (1.2.6)]), and by [8, Thm. 4.1] matroid polytopes in the hypersimplex $\Delta(k, n)$ correspond to matroids of rank $k$ on $\{1, \ldots, n\}$. In the theory of GIT $\Theta(V)$ corresponds to the stability set of $V$ denoted $\Omega(V)$ in [25].

Considering the quiver $P_{n}$ we have the representation space $R\left(P_{n}\right)=\left(\mathbb{A}^{1} \times \mathbb{A}^{1}\right)^{n}$ with the operation of $\left(\mathbb{G}_{m} \times \mathbb{G}_{m}\right) \times\left(\mathbb{G}_{m}\right)^{n}$. We write a free representation of $P_{n}$ over a scheme $Y$ after a choice of a basis as a tuple $V=\left(s_{1}, \ldots, s_{n}\right)$ of sections of $\left(\mathbb{A}^{1} \times \mathbb{A}^{1}\right)_{Y} \rightarrow Y$ where $s_{i}$ corresponds to $\left(\alpha_{i, 1}, \alpha_{i, 2}\right)$. We set $s_{i} \sim s_{j}$ if $s_{i}$ and $s_{j}$ are in the same $\mathbb{G}_{m}$-orbit and add the sections $s_{0}=(0,1), s_{\infty}=(1,0)$ which are only used to compare with sections $s_{i}$ up to $\sim$.

REMARK 2.4. Decomposition of the weight space for $P_{n}$.

(1) A weight for the quiver $P_{n}$ is an element $\theta=\left(\eta_{1}, \eta_{2}, \theta_{1}, \ldots, \theta_{n}\right)$ where $\theta_{p_{i}}=\eta_{i}$, $\theta_{q_{i}}=\theta_{i}$. We also write this as $\theta=\eta_{1} f_{1}+\eta_{2} f_{2}+\sum_{i} \theta_{i} e_{i}$ with $f_{1}, f_{2}, e_{1}, \ldots, e_{n}$ a basis of $\mathbb{Q}^{n+2}$. The weight space is the $(n+1)$-dimensional hyperplane $H\left(P_{n}\right)=\left\{\sum_{i} \theta_{i}=-\eta_{1}-\eta_{2}\right\} \subset \mathbb{Q}^{n+2}$. A semistable representation exists if and only if $\eta_{1}, \eta_{2} \leqslant 0$ and $\theta_{1}, \ldots, \theta_{n} \geqslant 0$. This defines a full-dimensional cone 
$C\left(P_{n}\right) \subset H\left(P_{n}\right)$. The affine hyperplane defined by $\eta_{1}+\eta_{2}=-1$ intersects this cone in a product of simplices

$$
\begin{aligned}
C\left(P_{n}\right) \cap\left\{\eta_{1}+\eta_{2}=-1\right\} & =\operatorname{conv}\left\{e_{1}-f_{1}, \ldots, e_{n}-f_{1}, e_{1}-f_{2}, \ldots, e_{n}-f_{2}\right\} \\
& =\Delta^{1} \times \Delta^{n-1} .
\end{aligned}
$$

(2) The outer walls are of the form $W_{q_{i}}=\left\{\theta_{i}=0\right\} \cap\left(\Delta^{1} \times \Delta^{n-1}\right) \cong \Delta^{1} \times \Delta^{n-2}$ and $W_{p_{j}}=\left\{\eta_{j}=0\right\} \cap\left(\Delta^{1} \times \Delta^{n-1}\right) \cong \Delta^{n-1}$. The inner walls are

$$
\begin{aligned}
W_{J} & =\left\{\theta \in \Delta^{1} \times \Delta^{n-1} \mid \sum_{i \in J} \theta_{i}=-\eta_{1}\right\} \\
& =\operatorname{conv}\left(\left\{e_{i}-f_{1} \mid i \in J\right\} \cup\left\{e_{i}-f_{2} \mid i \in J^{\complement}\right\}\right)
\end{aligned}
$$

for $J \subset\{1, \ldots, n\}, 1 \leqslant|J| \leqslant n-1, n \geqslant 2$. It is $W_{J} \cong \Delta^{n-1}$.

(3) An inner wall $W_{J}$ intersects the boundary of $\Delta^{1} \times \Delta^{n-1}$ as follows: $W_{J} \cap$ $W_{q_{i}}=\operatorname{conv}\left(\left\{e_{j}-f_{1} \mid j \in J \backslash\{i\}\right\} \cup\left\{e_{j}-f_{2} \mid j \in J^{\complement} \backslash\{i\}\right\}\right)$ (i.e. a wall $\left.W_{J \backslash\{i\}} \subset \Delta^{1} \times \Delta^{n-2}\right)$ and $W_{J} \cap W_{q_{1}}=\operatorname{conv}\left(\left\{e_{i}-f_{2} \mid i \in J^{\complement}\right\}\right)=\Delta^{\left|J^{\complement}\right|-1}$, $W_{J} \cap W_{q_{2}}=\operatorname{conv}\left(\left\{e_{i}-f_{1} \mid i \in J\right\}\right)=\Delta^{|J|-1}$.

(4) Two inner walls $W_{J_{1}}, W_{J_{2}}$ do not intersect in the interior of $\Delta^{1} \times \Delta^{n-1}$ if and only if $J_{1} \subsetneq J_{2}$ or $J_{2} \subsetneq J_{1}$

REMARK 2.5. The notation is such that for $\tau \in \operatorname{relint}\left(W_{J}\right)$ representations $\left(s_{1}, \ldots, s_{n}\right)$ with $s_{j} \sim s_{\infty}=(1,0)$ for $j \in J$ and $s_{i} \sim s_{0}=(0,1)$ for $i \in J^{\complement}$ are strictly $\tau$-semistable.

The following lemma can be proven in the same way as lemma 2.3 using remark 2.4.

LEMmA 2.6. Let $V=\left(s_{1}, \ldots, s_{n}\right)$ be a representation of $P_{n}$ over an algebraically closed field that is semistable with respect to some weight in the interior of $\Delta^{1} \times \Delta^{n-1}$, in particular all $s_{i}$ are nonzero. Let $J_{0}, J_{\infty} \subset\{1, \ldots, n\}$ be defined by

$$
J_{0}=\left\{i \mid s_{i} \sim s_{0}=(0,1)\right\}, J_{\infty}=\left\{i \mid s_{i} \sim s_{\infty}=(1,0)\right\} .
$$

The set $\Theta(V)=\left\{\theta \in \Delta^{1} \times \Delta^{n-1} \mid V\right.$ is $\theta$-semistable $\}$ is a closed convex polytope in $\Delta^{1} \times \Delta^{n-1}$ with the following properties:

(a) $\Theta(V)=\left\{\theta \in \Delta^{1} \times \Delta^{n-1} \mid \sum_{i \in J_{0}} \theta_{i} \leqslant-\eta_{2}\right\} \cap\left\{\theta \in \Delta^{1} \times \Delta^{n-1} \mid \sum_{i \in J_{\infty}} \theta_{i} \leqslant\right.$ $\left.-\eta_{1}\right\}$, i.e. $\Theta(V)$ is bounded within $\Delta^{1} \times \Delta^{n-1}$ by the walls $W_{J_{0}^{\mathrm{c}}}, W_{J_{\infty}}$. These walls, if distinct, do not intersect in the interior of $\Delta^{1} \times \Delta^{n-1}$.

(b) The set of vertices of $\Theta(V)$ is a subset of the set of vertices of $\Delta^{1} \times \Delta^{n-1}$. It is $\Theta(V)=\operatorname{conv}\left(\left\{e_{i}-f_{2} \mid i \in J_{0}\right\} \cup\left\{e_{i}-f_{1} \mid i \in J_{\infty}\right\}\right)$.

(c) $\Theta(V)$ is full dimensional if and only if $\left(J_{0} \cup J_{\infty}\right)^{\complement} \neq \varnothing$, or equivalently, $V$ is stable with respect to some weight. Otherwise $\Theta(V)=W_{J_{0}^{\mathrm{C}}}=W_{J_{\infty}}$.

2.2. Relation Between Representations of $Q_{n+2}$ And $P_{n}$. We show that the structure of GIT equivalence classes in $\Delta^{1} \times \Delta^{n-1} \subset H\left(P_{n}\right)$ arises from the structure of GIT equivalence classes in $\Delta(2, n+2)$ in the neighbourhood of some vertex $e_{a}+e_{b}$ and compare the corresponding stacks and moduli spaces of representations.

The subset $\Delta(2, n+2)_{a, b}=\Delta(2, n+2) \cap\left\{\theta_{a}+\theta_{b} \geqslant \sum_{i \neq a, b} \theta_{i}\right\} \backslash\left\{e_{a}+e_{b}\right\}$ is part of a cone with apex $e_{a}+e_{b}$ and we can project it onto $W_{\left\{\{a, b\},\{a, b\}^{\mathrm{C}}\right\}}=$ $\Delta(2, n+2) \cap\left\{\theta_{a}+\theta_{b}=\sum_{i \neq a, b} \theta_{i}\right\}$ by

$$
\begin{aligned}
\Delta(2, n+2)_{a, b} & \rightarrow W_{\left\{\{a, b\},\{a, b\}^{\complement}\right\}} \\
\theta=\left(\theta_{a}, \theta_{b},\left(\theta_{i}\right)_{i \neq a, b}\right) & \mapsto \bar{\theta}=\left(\lambda\left(\theta_{a}-1\right)+1, \lambda\left(\theta_{b}-1\right)+1,\left(\lambda \theta_{i}\right)_{i \neq a, b}\right)
\end{aligned}
$$

where $\lambda=1 / \sum_{i \neq a, b} \theta_{i}$. The wall $W_{\left\{\{a, b\},\{a, b\}^{0}\right\}}=\operatorname{conv}\left(\left\{e_{i}+e_{a} \mid i \neq a, b\right\} \cup\left\{e_{i}+\right.\right.$ $\left.\left.e_{b} \mid i \neq a, b\right\}\right) \subset \Delta(2, n+2)$ can be identified with $\Delta^{1} \times \Delta^{n-1}=\operatorname{conv}\left(\left\{e_{i}-f_{2} \mid i \neq\right.\right.$ 
$\left.a, b\} \cup\left\{e_{i}-f_{1} \mid i \neq a, b\right\}\right) \subset H\left(P_{n}\right)$ by

$$
\begin{aligned}
W_{\left\{\{a, b\},\{a, b\}^{\mathrm{C}}\right\}} & \rightarrow \Delta^{1} \times \Delta^{n-1} \subset H\left(P_{n}\right) \\
\bar{\theta}=\left(\bar{\theta}_{a}, \bar{\theta}_{b},\left(\bar{\theta}_{i}\right)_{i \neq a, b}\right) & \mapsto \theta^{\prime}=\left(\eta_{1}^{\prime}=\bar{\theta}_{a}-1, \eta_{2}^{\prime}=\bar{\theta}_{b}-1,\left(\theta_{i}^{\prime}=\bar{\theta}_{i}\right)_{i \neq a, b}\right)
\end{aligned}
$$

We observe that the projections of the walls of $\Delta(2, n+2)$ near $e_{a}+e_{b}$ coincide with the walls of $\Delta^{1} \times \Delta^{n-1} \subset H\left(P_{n}\right)$. The walls meeting the interior of $\Delta(2, n+2)_{a, b}$ are the walls $W_{\left\{J, J^{\complement}\right\}}$ with $a \in J, b \in J^{\complement}$ or $a \in J^{\complement}, b \in J$. Their intersections with $W_{\left\{\{a, b\},\{a, b\}^{\complement}\right\}}$ are exactly the inner walls of $\Delta^{1} \times \Delta^{n-1}$ : assume $a \in J, b \in J^{\complement}$, then the equation $\sum_{j \in J} \theta_{j}=1$ for $W_{\left\{J, J^{\complement}\right\}}$ is equivalent to the equation $\sum_{j \in J \backslash\{a\}} \theta_{j}^{\prime}=-\eta_{1}^{\prime}$ defining $W_{J \backslash\{a\}} \subset \Delta^{1} \times \Delta^{n-1}$.

Proposition 2.7. Let $a, b \in\{1, \ldots, n+2\}, a \neq b$.

(a) The maps

$$
\begin{aligned}
& \left\{\begin{array}{l}
\text { free representations with fixed bases } \\
V=\left(s_{1}, \ldots, s_{n+2}\right) \text { of } Q_{n+2} \text { over } Y \\
\text { such that } s_{a} \cap 0=s_{b} \cap 0=\varnothing \\
\text { and } \mathbb{G}_{m} s_{a} \cap \mathbb{G}_{m} s_{b}=\varnothing
\end{array}\right\} \rightarrow\left\{\begin{array}{l}
\text { free representations } \\
\text { with fixed bases of } P_{n} \\
\text { over } Y
\end{array}\right\} \\
& V=\left(s_{1}, \ldots, s_{n+2}\right) \mapsto V^{\prime}=\left(s_{i}^{\prime}\right)_{i \neq a, b}
\end{aligned}
$$

for schemes $Y$ over a base scheme $S$, where $s_{i}^{\prime}$ arises from $s_{i}$ by the base change that transforms $s_{a}$ to $(1,0)$ and $s_{b}$ to $(0,1)$, induce an isomorphism of stacks

$$
\begin{aligned}
{\left[R\left(Q_{n+2}\right)_{a, b} /((\operatorname{GL}(2) \times\right.} & \left.\left.\left.\left(\mathbb{G}_{m}\right)^{n}\right) / \mathbb{G}_{m}\right)\right] \\
& \left.\rightarrow\left[R\left(P_{n}\right) /\left(\left(\mathbb{G}_{m} \times \mathbb{G}_{m}\right) \times\left(\mathbb{G}_{m}\right)^{n}\right) / \mathbb{G}_{m}\right)\right]
\end{aligned}
$$

where $R\left(Q_{n+2}\right)_{a, b}=R\left(Q_{n+2}\right) \backslash\left(\left\{s_{a}=0\right\} \cup\left\{s_{b}=0\right\} \cup\left\{\mathbb{G}_{m} s_{a}=\mathbb{G}_{m} s_{b}\right\}\right)$.

(b) We have the map

$$
\begin{aligned}
\Delta(2, n+2)_{a, b} & \rightarrow \Delta^{1} \times \Delta^{n-1} \subset H\left(P_{n}\right) \\
\theta & \mapsto \theta^{\prime}=\left(\eta_{1}^{\prime}=\lambda\left(\theta_{a}-1\right), \eta_{2}^{\prime}=\lambda\left(\theta_{b}-1\right),\left(\theta_{i}^{\prime}=\lambda \theta_{i}\right)_{i \neq a, b}\right)
\end{aligned}
$$

where $\lambda=1 / \sum_{i \neq a, b} \theta_{i}$. On the fibres of this map the $\theta$-(semi)stable locus in $R\left(Q_{n+2}\right)_{a, b}$ does not change.

(c) Let $\theta \in W_{\left\{\{a, b\},\{a, b\}^{\complement}\right\}} \subset \Delta(2, n+2)$ and $\theta^{\prime} \in \Delta^{1} \times \Delta^{n-1} \subset H\left(P_{n}\right)$ its image under the map (4). Let $l \in \mathbb{N}_{>0}$ such that $l \theta$ and $l \theta^{\prime}$ are integral. Then the equivariant line bundles $\mathcal{O}_{R\left(Q_{n+2}\right)_{a, b}}$ with $l \theta$-linearisation and $\mathcal{O}_{R\left(P_{n}\right)}$ with $l \theta^{\prime}$ linearisation define isomorphic line bundles on the quotient stacks under the identification (3).

Proof. (a) The maps on the sets of morphisms are given by composition with the base change maps that transform $s_{a}, s_{b}$ to $(1,0),(0,1)$ and forgetting the automorphisms of the spaces corresponding to the vertices $q_{a}, q_{b}$. The inverse functor is given on the objects by maps $\left(s_{i}\right)_{i \neq a, b} \mapsto\left(s_{i}\right)_{i}$ setting $s_{a}=(1,0), s_{b}=(0,1)$ and on the morphisms by taking those automorphisms of the spaces corresponding to the vertices $q_{a}, q_{b}$ such that $s_{a}, s_{b}$ remain fixed. One checks that both compositions of these two functors are isomorphic to the identity functors.

(b) The fibres are the line segments $\left[\bar{\theta}, e_{a}+e_{b}\right] \backslash\left\{e_{a}+e_{b}\right\}$ for $\bar{\theta} \in W_{\left\{\{a, b\},\{a, b\}^{\complement}\right\}}$. All $\theta$ contained in this line segment are elements of the same GIT equivalence class except $\bar{\theta}$. But $R^{\bar{\theta}}\left(Q_{n+2}\right)$ differs from $R^{\theta}\left(Q_{n+2}\right)$ for the other $\theta$ only outside $R\left(Q_{n+2}\right)_{a, b}$.

(c) The isomorphism (3) identifies the structure sheaves of the two stacks. We have to verify that the additional multiplications by the corresponding characters 
coincide. It suffices to consider the groupoids of $Y$-valued points for $S$-schemes $Y$ of the two given groupoid schemes. The elements of $\left(\left(\mathrm{GL}(2) \times\left(\mathbb{G}_{m}\right)^{n}\right) / \mathbb{G}_{m}\right)(Y)$ fixing $s_{a}=(1,0), s_{b}=(0,1)$ are of the form $\left(\left(\begin{array}{cc}\alpha_{a} & 0 \\ 0 & \alpha_{b}\end{array}\right), \alpha_{a}, \alpha_{b},\left(\alpha_{i}\right)_{i \neq a, b}\right)$, the corresponding element $\left.\left(\beta_{1}, \beta_{2},\left(\alpha_{i}\right)_{i \neq a, b}\right) \in\left(\left(\mathbb{G}_{m} \times \mathbb{G}_{m}\right) \times\left(\mathbb{G}_{m}\right)^{n}\right) / \mathbb{G}_{m}\right)(Y)$ satisfies $\beta_{1}=\alpha_{a}$, $\beta_{2}=\alpha_{b}$. Applying the character corresponding to $l \theta=\left(l \eta, l \theta_{1}, \ldots, l \theta_{n+2}\right)$ we obtain $\left(\alpha_{a} \alpha_{b}\right)^{l \eta} \alpha_{a}^{l \theta_{a}} \alpha_{b}^{l \theta_{b}} \prod_{i \neq a, b} \alpha_{i}^{l \theta_{i}}$ and applying $l \theta^{\prime}$ we have $\beta_{1}^{l \eta_{1}^{\prime}} \beta_{2}^{l \eta_{2}^{\prime}} \prod_{i \neq a, b} \alpha_{i}^{l \theta_{i}^{\prime}}$. As $\eta=-1$, this coincides for $\theta_{i}^{\prime}=\theta_{i}, \eta_{1}^{\prime}=\theta_{a}-1, \eta_{2}^{\prime}=\theta_{b}-1$.

Corollary 2.8. Let $a, b \in\{1, \ldots, n+2\}, a \neq b$.

(a) For $\theta \in \Delta(2, n+2)_{a, b} \backslash W_{\left\{\{a, b\},\{a, b\}^{\circ}\right\}}$ a free representation $V=\left(s_{1}, \ldots, s_{n+2}\right)$ of $Q_{n+2}$ is $\theta$-(semi)stable if and only if $V$ satisfies $s_{a} \cap 0=\varnothing, s_{b} \cap 0=\varnothing$, $\mathbb{G}_{m} s_{a} \cap \mathbb{G}_{m} s_{b}=\varnothing$ and its image under (2) is a $\theta^{\prime}$-(semi)stable representation of $P_{n}$, where $\theta^{\prime}$ is the image of $\theta$ under the map (4).

(b) The map (4) defines a bijection between the GIT equivalence classes in $\Delta^{1} \times$ $\Delta^{n-1}$ and in $\Delta(2, n+2)_{a, b} \backslash W_{\left\{\{a, b\},\{a, b\}^{c}\right\}}\left(\right.$ resp. $\left.W_{\left\{\{a, b\},\{a, b\}^{\mathrm{C}}\right\}}\right)$.

(c) For $\theta \in \Delta(2, n+2)_{a, b} \backslash W_{\left\{\{a, b\},\{a, b\}^{\complement}\right\}}$ the isomorphism (3) induces an isomorphism of stacks

$$
\begin{aligned}
{\left[R^{\theta}\left(Q_{n+2}\right) /((\operatorname{GL}(2)\right.} & \left.\left.\left.\times\left(\mathbb{G}_{m}\right)^{n}\right) / \mathbb{G}_{m}\right)\right] \\
& \left.\rightarrow\left[R^{\theta^{\prime}}\left(P_{n}\right) /\left(\left(\mathbb{G}_{m} \times \mathbb{G}_{m}\right) \times\left(\mathbb{G}_{m}\right)^{n}\right) / \mathbb{G}_{m}\right)\right]
\end{aligned}
$$

and thus an isomorphism of their moduli spaces

$$
\mathcal{M}^{\theta}\left(Q_{n+2}\right) \rightarrow \mathcal{M}^{\theta^{\prime}}\left(P_{n}\right) .
$$

These isomorphisms determine an isomorphism of the inverse systems of the moduli spaces $\mathcal{M}^{\theta}\left(Q_{n+2}\right)$ for $\theta \in \Delta(2, n+2)_{a, b} \backslash W_{\left\{\{a, b\},\{a, b\}^{\complement}\right\}}$ and $\mathcal{M}^{\theta^{\prime}}\left(P_{n}\right)$ for $\theta^{\prime} \in \Delta^{1} \times \Delta^{n-1}$.

2.3. Moduli spaces for $P_{n}, Q_{n}$ ANd GIT quotients of PROduCts of $\mathbb{P}^{1}$ s. We compare the moduli spaces of representations of $Q_{n}$ and $P_{n}$ to GIT quotients of $\left(\mathbb{P}^{1}\right)^{n}$ by the quotient groups $\left(\left(\mathrm{GL}(2) \times\left(\mathbb{G}_{m}\right)^{n}\right) / \mathbb{G}_{m}\right) /\left(\left(\mathbb{G}_{m} \times\left(\mathbb{G}_{m}\right)^{n}\right) / \mathbb{G}_{m}\right) \cong \operatorname{PGL}(2)$ and $\left(\left(\left(\mathbb{G}_{m}\right)^{2} \times\left(\mathbb{G}_{m}\right)^{n}\right) / \mathbb{G}_{m}\right) /\left(\left(\mathbb{G}_{m} \times\left(\mathbb{G}_{m}\right)^{n}\right) / \mathbb{G}_{m}\right) \cong \mathbb{G}_{m}$.

We have $\mathrm{NS}\left(\left(\mathbb{P}^{1}\right)^{n}\right)=\operatorname{Pic}\left(\left(\mathbb{P}^{1}\right)^{n}\right) \cong \mathbb{Z}^{n}$, where $\left(\theta_{1}, \ldots, \theta_{n}\right) \in \mathbb{Z}^{n}$ corresponds to the line bundle $\mathcal{O}_{\left(\mathbb{P}^{1}\right)^{n}}\left(\theta_{1}, \ldots, \theta_{n}\right)=\mathcal{O}_{\mathbb{P}^{1}}\left(\theta_{1}\right) \otimes \ldots \otimes \mathcal{O}_{\mathbb{P}^{1}}\left(\theta_{n}\right)$. A line bundle $\mathcal{O}_{\left(\mathbb{P}^{1}\right)^{n}}\left(\theta_{1}, \ldots, \theta_{n}\right)$ with $\theta_{i} \in 2 \mathbb{Z}$ for all $i$ has a $\operatorname{PGL}(2)$-linearisation $\left(\mathcal{O}_{\mathbb{P}^{1}}(-2)\right.$ being the canonical sheaf of $\left.\mathbb{P}^{1}\right)$, and if an element of $\operatorname{Pic}\left(\left(\mathbb{P}^{1}\right)^{n}\right)$ has a $\operatorname{PGL}(2)$-linearisation then this linearisation is unique. Thus we can identify $\operatorname{NS}^{\mathrm{PGL}(2)}\left(\left(\mathbb{P}^{1}\right)^{n}\right)_{\mathbb{Q}}=$ $\operatorname{Pic}^{\mathrm{PGL}(2)}\left(\left(\mathbb{P}^{1}\right)^{n}\right)_{\mathbb{Q}} \cong \mathbb{Q}^{n}$ with $H\left(Q_{n}\right)$ by $\left(\theta_{1}, \ldots, \theta_{n}\right) \leftrightarrow\left(\eta=-\frac{1}{2} \sum_{i} \theta_{i}, \theta_{1}, \ldots, \theta_{n}\right)$.

Proposition 2.9. Let $R^{*}\left(Q_{n}\right)=R\left(Q_{n}\right) \backslash \bigcup_{i}\left\{s_{i}=0\right\} \cong\left(\mathbb{A}^{2} \backslash\{0\}\right)^{n}$.

(a) The morphism $R^{*}\left(Q_{n}\right) \rightarrow\left(\mathbb{P}^{1}\right)^{n}$ induces an isomorphism of stacks

$$
\left[R^{*}\left(Q_{n}\right) /\left(\left(\mathrm{GL}(2) \times\left(\mathbb{G}_{m}\right)^{n}\right) / \mathbb{G}_{m}\right)\right] \rightarrow\left[\left(\mathrm{P}^{1}\right)^{n} / \mathrm{PGL}(2)\right] .
$$

(b) Let $\theta=\left(\eta, \theta_{1}, \ldots, \theta_{n}\right) \in H\left(Q_{n}\right)$ be integral. Then the line bundle $\mathcal{O}_{\left(\mathbb{P}^{1}\right)^{n}}\left(\theta_{1}, \ldots, \theta_{n}\right)$ has a (unique) $\mathrm{PGL}(2)$-linearisation and the equivariant line bundles $\mathcal{O}_{R^{*}\left(Q_{n}\right)}$ with $\theta$-linearisation and $\mathcal{O}_{\left(\mathbb{P}^{1}\right)^{n}}\left(\theta_{1}, \ldots, \theta_{n}\right)$ define isomorphic line bundles on the quotient stacks under the identification in (5).

Proof. Consider the operation of the subgroup $\left(\mathbb{G}_{m}\right)^{n} \cong\left(\mathbb{G}_{m} \times\left(\mathbb{G}_{m}\right)^{n}\right) / \mathbb{G}_{m} \subset$ $\left(\mathrm{GL}(2) \times\left(\mathbb{G}_{m}\right)^{n}\right) / \mathbb{G}_{m}$ on $R^{*}\left(Q_{n}\right) \cong\left(\mathbb{A}^{2} \backslash\{0\}\right)^{n}$. The operation of $\mathbb{G}_{m}$ on $\mathbb{A}^{2} \backslash\{0\}$ is free and $\mathbb{A}^{2} \backslash\{0\} \rightarrow \mathbb{P}^{1}$ a $\mathbb{G}_{m}$-torsor. The structure sheaf on $\mathbb{A}^{2} \backslash\{0\}$ with $\mathbb{G}_{m^{-}}$ linearisation by $\theta$ descends to the line bundle $\mathcal{O}_{\mathbb{P}^{1}}(\theta)$. Thus $R^{*}\left(Q_{n}\right) \rightarrow\left(\mathbb{P}^{1}\right)^{n}$ is a 
$\left(\mathbb{G}_{m}\right)^{n}$-torsor and $\mathcal{O}_{R^{*}\left(Q_{n}\right)}$ with $\left(\mathbb{G}_{m}\right)^{n}$-linearisation by $\left(\theta_{1}, \ldots, \theta_{n}\right)$ descends to the line bundle $\mathcal{O}_{\left(\mathbb{P}^{1}\right)^{n}}\left(\theta_{1}, \ldots, \theta_{n}\right)$.

(a) A $Y$-valued point of $\left[R^{*}\left(Q_{n}\right) /\left(\left(\mathrm{GL}(2) \times\left(\mathbb{G}_{m}\right)^{n}\right) / \mathbb{G}_{m}\right)\right]$ for some $S$-scheme $Y$ corresponds to a $\left(\mathrm{GL}(2) \times\left(\mathbb{G}_{m}\right)^{n}\right) / \mathbb{G}_{m}$-torsor $E \rightarrow Y$ with an equivariant morphism $E \rightarrow R^{*}\left(Q_{n}\right)$. Taking quotients by $\left(\mathbb{G}_{m}\right)^{n} \subset\left(\mathrm{GL}(2) \times\left(\mathbb{G}_{m}\right)^{n}\right) / \mathbb{G}_{m}$ we obtain a $\operatorname{PGL}(2)$-equivariant morphism $E /\left(\mathbb{G}_{m}\right)^{n} \rightarrow\left(\mathbb{P}^{1}\right)^{n}$ where $E /\left(\mathbb{G}_{m}\right)^{n} \rightarrow Y$ is a PGL(2)torsor. The diagram

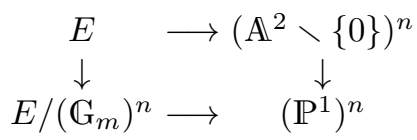

is cartesian and its vertical arrows are $\left(\mathbb{G}_{m}\right)^{n}$-torsors. For a $Y$-valued point of $\left[\left(\mathbb{P}^{1}\right)^{n} / \mathrm{PGL}(2)\right]$, a PGL(2)-torsor $\bar{E} \rightarrow Y$ with equivariant morphism to $\left(\mathbb{P}^{1}\right)^{n}$, we construct a $\left(\mathrm{GL}(2) \times\left(\mathbb{G}_{m}\right)^{n}\right) / \mathbb{G}_{m}$-torsor over $Y$ with an equivariant morphism to $\left(\mathbb{A}^{2} \backslash\{0\}\right)^{n}$ by taking the fibred product $\bar{E} \times\left(\mathbb{P}^{1}\right)^{n}\left(\mathbb{A}^{2} \backslash\{0\}\right)^{n}$. One verifies that this way two functors between $\left[R^{*}\left(Q_{n}\right) /\left(\left(\mathrm{GL}(2) \times\left(\mathbb{G}_{m}\right)^{n}\right) / \mathbb{G}_{m}\right)\right]$ and $\left[\left(\mathbb{P}^{1}\right)^{n} / \operatorname{PGL}(2)\right]$ are defined whose compositions are isomorphic to the identity functors.

(b) As the categories of sheaves do not change under stackification we may work with the prestack $\left[R^{*}\left(Q_{n}\right) /\left(\left(\mathrm{GL}(2) \times\left(\mathbb{G}_{m}\right)^{n}\right) / \mathbb{G}_{m}\right)\right]^{\text {pre }}$ of trivial $\left(\mathrm{GL}(2) \times\left(\mathbb{G}_{m}\right)^{n}\right) / \mathbb{G}_{m^{-}}$ torsors $E \rightarrow Y$ with an equivariant morphism $E \rightarrow R^{*}\left(Q_{n}\right)$, and its image in $\left[\left(\mathbb{P}^{1}\right)^{n} / \mathrm{PGL}(2)\right]$ under the isomorphism (5). Objects in $\left[R^{*}\left(Q_{n}\right) /((\mathrm{GL}(2) \times\right.$ $\left.\left.\left.\left(\mathbb{G}_{m}\right)^{n}\right) / \mathbb{G}_{m}\right)\right]^{\text {pre }}$ over an $S$-scheme $Y$ correspond to morphisms $Y \rightarrow R^{*}\left(Q_{n}\right)$, a morphism $(g, y): Y \rightarrow\left(\mathrm{GL}(2) \times\left(\mathbb{G}_{m}\right)^{n}\right) / \mathbb{G}_{m} \times R^{*}\left(Q_{n}\right)$ gives an arrow $y \rightarrow g y$ over $i d_{Y}$.

Let $\mathscr{L}$ be the line bundle on $\left[R^{*}\left(Q_{n}\right) /\left(\left(\mathrm{GL}(2) \times\left(\mathbb{G}_{m}\right)^{n}\right) / \mathbb{G}_{m}\right)\right]^{\text {pre }}$ defined by the equivariant line bundle $\mathcal{O}_{R^{*}\left(Q_{n}\right)}$ with linearisation by $\theta$, in particular $\mathscr{L}(y)=\Gamma\left(Y, y^{*} \mathcal{O}_{R^{*}\left(Q_{n}\right)}\right)$ for $y: Y \rightarrow R^{*}\left(Q_{n}\right)$. We describe the push-forward $\overline{\mathscr{L}}$ with respect to the isomorphism (5), which by definition is given by $\overline{\mathscr{L}}(\bar{y})=\lim _{\overline{y^{\prime}} \rightarrow \bar{y}} \mathscr{L}\left(y^{\prime}\right)$ where $\overline{y^{\prime}}$ is the image of the object $y^{\prime}$ under (5). For open embeddings $\bar{y}: \bar{U} \hookrightarrow\left(\mathbb{P}^{1}\right)^{n}$ and $y: U \hookrightarrow R^{*}\left(Q_{n}\right)$ such that $U \subseteq R^{*}\left(Q_{n}\right)$ is the preimage of $\bar{U} \subseteq\left(\mathbb{P}^{1}\right)^{n}$ we have $\overline{\mathscr{L}}(\bar{y})=\lim _{\overline{y^{\prime}} \rightarrow \bar{y}} \mathscr{L}\left(y^{\prime}\right)=\lim _{h \in\left(\mathbb{G}_{m}\right)^{n}(U), \overline{h y} \rightarrow \bar{y}} \mathscr{L}(h y)=\Gamma\left(U, \mathcal{O}_{R^{*}\left(Q_{n}\right)}\right)^{\left(\mathbb{G}_{m}\right)^{n}}=$ $\Gamma\left(\bar{U}, \mathcal{O}_{\left(\mathbb{P}^{1}\right)^{n}}\left(\theta_{1}, \ldots, \theta_{n}\right)\right)$. Further we have the natural restriction maps. Thus the line bundle $\overline{\mathscr{L}}$ comes from the line bundle $\mathcal{O}_{\left(\mathbb{P}^{1}\right)^{n}}\left(\theta_{1}, \ldots, \theta_{n}\right)$ on $\left(\mathbb{P}^{1}\right)^{n}$ with some PGL(2)-linearisation.

COROLlary 2.10

(a) We have an identification $\operatorname{Pic}^{\mathrm{PGL}(2)}\left(\left(\mathbb{P}^{1}\right)^{n}\right) \cong H\left(Q_{n}\right) \cap \mathbb{Z}^{n+1}$ by $\left(\theta_{1}, \ldots, \theta_{n}\right) \leftrightarrow$ $\left(\eta=-\frac{1}{2} \sum_{i} \theta_{i}, \theta_{1}, \ldots, \theta_{n}\right)$.

(b) For $\left(\eta, \theta_{1}, \ldots, \theta_{n}\right) \in H\left(Q_{n}\right)$ such that $\theta_{i}>0$ for all $i$ a free representation $V=\left(s_{1}, \ldots, s_{n}\right)$ of $Q_{n}$ is $\left(\eta, \theta_{1}, \ldots, \theta_{n}\right)$-(semi)stable if and only if $s_{i} \cap 0=\varnothing$ for all $i$ and its image under $(5)$ is a $\left(\theta_{1}, \ldots, \theta_{n}\right)$-(semi) stable section of $\left(\mathbb{P}^{1}\right)^{n}$.

(c) The image of int $C\left(Q_{n}\right) \subset H\left(Q_{n}\right)$ under the isomorphism $\operatorname{Pic}^{\operatorname{PGL}(2)}\left(\left(\mathbb{P}^{1}\right)^{n}\right)_{\mathbb{Q}} \cong$ $H\left(Q_{n}\right)$ is the $\mathrm{PGL}(2)$-ample cone in $\mathrm{Pic}^{\mathrm{PGL}(2)}\left(\left(\mathbb{P}^{1}\right)^{n}\right)_{\mathrm{Q}}$. The GIT classes in these open cones coincide.

(d) For $\theta \in H\left(Q_{n}\right) \cong \operatorname{Pic}^{\mathrm{PGL}(2)}\left(\left(\mathbb{P}^{1}\right)^{n}\right)_{\mathbb{Q}}$ such that $\theta_{i}>0$ for all $i$ the isomorphism (5) induces isomorphisms of the stacks of $\theta$-(semi)stable points, of their moduli spaces and their inverse systems.

One can also compare these stacks with $\left[G(2, n) /\left(\left(\mathbb{G}_{m}\right)^{n} / \mathbb{G}_{m}\right)\right]$, cf. $[17,2.4]$.

Similarly, one can show the analogous results for the quiver $P_{n}$. In the case of the quiver $P_{n}$ we have $\mathrm{NS}^{\mathbb{G}_{m}}\left(\left(\mathbb{P}^{1}\right)^{n}\right)=\operatorname{Pic}^{\mathbb{G}_{m}}\left(\left(\mathbb{P}^{1}\right)^{n}\right) \cong \mathbb{Z}^{n+1}$, where $\left(\theta_{1}, \ldots, \theta_{n}\right) \in \mathbb{Z}^{n}$ 
defines an element $\mathcal{O}_{\left(\mathbb{P}^{1}\right)^{n}}\left(\theta_{1}, \ldots, \theta_{n}\right) \in \operatorname{Pic}\left(\left(\mathbb{P}^{1}\right)^{n}\right)$ and for each $\mathcal{O}_{\left(\mathbb{P}^{1}\right)^{n}}\left(\theta_{1}, \ldots, \theta_{n}\right)$ the set of $\mathbb{G}_{m}$-linearisations is a principal homogeneous space under the character group $\mathbb{Z}$ of $\mathbb{G}_{m}$. Below we identify $\operatorname{Pic}^{\mathbb{G}_{m}}\left(\left(\mathbb{P}^{1}\right)^{n}\right)$ with $H\left(P_{n}\right) \cap \mathbb{Z}^{n+2}$, where $\left(\eta_{1}, \eta_{2}, \theta_{1}, \ldots, \theta_{n}\right) \in H\left(P_{n}\right) \cap \mathbb{Z}^{n+2}$ corresponds to the line bundle $\mathcal{O}_{\left(\mathbb{P}^{1}\right)^{n}}\left(\theta_{1}, \ldots, \theta_{n}\right)$ with a certain $\mathbb{G}_{m}$-linearisation, and the $\mathbb{G}_{m^{m}}$-linearisations on $\mathcal{O}_{\left(\mathbb{P}^{1}\right)^{n}}\left(\theta_{1}, \ldots, \theta_{n}\right)$ corresponding to $\left(\eta_{1}, \eta_{2}, \theta_{1}, \ldots, \theta_{n}\right)$ and $\left(\eta_{1}^{\prime}, \eta_{2}^{\prime}, \theta_{1}, \ldots, \theta_{n}\right)$ differ by the character $(\eta,-\eta)=$ $\left(\eta_{1}-\eta_{1}^{\prime}, \eta_{2}-\eta_{2}^{\prime}\right)$ of $\left(\mathbb{G}_{m}\right)^{2} / \mathbb{G}_{m} \cong \mathbb{G}^{m}$.

Proposition 2.11. Let $R^{*}\left(P_{n}\right)=R\left(P_{n}\right) \backslash \bigcup_{i}\left\{s_{i}=0\right\} \cong\left(\mathbb{A}^{2} \backslash\{0\}\right)^{n}$.

(a) The morphism $R^{*}\left(P_{n}\right) \rightarrow\left(\mathbb{P}^{1}\right)^{n}$ induces an isomorphism of stacks

$$
\left[R^{*}\left(P_{n}\right) /\left(\left(\left(\mathbb{G}_{m}\right)^{2} \times\left(\mathbb{G}_{m}\right)^{n}\right) / \mathbb{G}_{m}\right)\right] \rightarrow\left[\left(\mathbb{P}^{1}\right)^{n} / \mathbb{G}_{m}\right]
$$

(b) Let $\left(\eta_{1}, \eta_{2}, \theta_{1}, \ldots, \theta_{n}\right) \in H\left(P_{n}\right)$ be integral. Then the line bundle $\mathcal{O}_{R^{*}\left(P_{n}\right)}$ with $\left(\left(\mathbb{G}_{m}\right)^{2} \times\left(\mathbb{G}_{m}\right)^{n}\right) / \mathbb{G}_{m}$-linearisation given by $\left(\eta_{1}, \eta_{2}, \theta_{1}, \ldots, \theta_{n}\right)$ descends to the line bundle $\mathcal{O}_{\left(\mathbb{P}^{1}\right)^{n}}\left(\theta_{1}, \ldots, \theta_{n}\right)$ with a certain $\mathbb{G}_{m}$-linearisation, and both define isomorphic line bundles on the quotient stacks under the identification in (6).

\section{COROllary 2.12 .}

(a) For $\theta=\left(\eta_{1}, \eta_{2}, \theta_{1}, \ldots, \theta_{n}\right) \in H\left(P_{n}\right)$ such that $\theta_{i}>0$ for all $i$ a free representation $V=\left(s_{1}, \ldots, s_{n}\right)$ of $P_{n}$ is $\theta$-(semi)stable if and only if $s_{i} \cap 0=\varnothing$ for all $i$ and its image under (5) is a (semi)stable section of $\left(\mathbb{P}^{1}\right)^{n}$ with respect to $\mathcal{O}_{\left(\mathbb{P}^{1}\right)^{n}}\left(\theta_{1}, \ldots, \theta_{n}\right)$ with the corresponding $\mathbb{G}_{m}$-linearisation.

(b) The image of int $C\left(P_{n}\right) \subset H\left(P_{n}\right)$ under the isomorphism $\operatorname{Pic}^{\mathbb{G}_{m}}\left(\left(\mathbb{P}^{1}\right)^{n}\right)_{\mathbb{Q}} \cong$ $H\left(P_{n}\right)$ is the $\mathbb{G}_{m}$-ample cone in $\operatorname{Pic}^{\mathbb{G}_{m}}\left(\left(\mathbb{P}^{1}\right)^{n}\right)_{\mathbb{Q}}$. The GIT classes in these open cones coincide.

(c) For $\theta \in H\left(P_{n}\right) \cong \operatorname{Pic}^{\mathbb{G}_{m}}\left(\left(\mathbb{P}^{1}\right)^{n}\right)_{\mathbb{Q}}$ such that $\theta_{i}>0$ for all $i$ the isomorphism (5) induces isomorphisms of the stacks of $\theta$-(semi)stable points, of their moduli spaces and their inverse systems.

In the following, when considering free representations $V=\left(s_{1}, \ldots, s_{n}\right)$ of $Q_{n}$ and $P_{n}$ such that $s_{i} \cap 0=\varnothing$ up to isomorphism, we will often write them as tuples of sections of $\left(\mathbb{P}^{1}\right)^{n}$.

2.4. The Functor of InVERSE Limits of QUiver VARIETIES For $P_{n}$ AND $Q_{n}$. Let $Q=Q_{n}$ or $Q=P_{n}$. Because all chambers are connected via the relative interiors of GIT equivalence classes of codimension one, we can rewrite the contravariant functor on $S$-schemes (1) in proposition 1.16 as

$$
\text { (7) } Y \mapsto\left\{\begin{array}{l|l}
\left(\varphi_{V^{\theta}}\right)_{\theta} \in \prod_{\theta \text { generic }} \mathcal{M}^{\theta}(Q)(Y) & \begin{array}{l}
\forall \theta, \theta^{\prime} \text { generic } \forall C_{\tau} \subset \operatorname{int} C(Q) \text { GIT } \\
\text { equiv. class of codimension one such that } \\
\bar{C}_{\tau} \subseteq \bar{C}_{\theta} \cap \bar{C}_{\theta^{\prime}}: \varphi_{\theta, \tau} \circ \varphi_{V^{\theta}}=\varphi_{\theta^{\prime}, \tau} \circ \varphi_{V^{\theta^{\prime}}}
\end{array}
\end{array}\right\}
$$

where the product is over representatives $\theta$ of the generic GIT equivalence classes and $\varphi_{V^{\theta}}: Y \rightarrow \mathcal{M}^{\theta}(Q)$ is the morphism determined by a $\theta$-stable representation $V^{\theta}$ over $Y$.

For a representation $V$ of $Q$ over an $S$-scheme $Y$ stable with respect to some generic $\theta$ the polytopes $\Theta(V(y))$ for the fibres $V(y)$ over the geometric points $y$ of $Y$ defined in subsection 2.1 have the property that $\left\{y^{\prime} \in Y \mid \Theta(V(y)) \subseteq \Theta\left(V\left(y^{\prime}\right)\right)\right\}$ are the points of an open subscheme of $Y$. Therefore for two representations $V, V^{\prime}$ we have an open subscheme

$$
U\left(V, V^{\prime}\right)=\left\{y \in Y \mid \operatorname{int}\left(\Theta(V(y)) \cap \Theta\left(V^{\prime}(y)\right)\right) \neq \varnothing\right\} \subseteq Y .
$$


If $V^{\theta}, V^{\theta^{\prime}}$ are part of a family over $Y$ satisfying the conditions in (7), then

$$
U\left(V^{\theta}, V^{\theta^{\prime}}\right)=\left\{y \in Y \mid V^{\theta}(y) \theta^{\prime} \text {-stable }\right\}=\left\{y \in Y \mid V^{\theta^{\prime}}(y) \theta \text {-stable }\right\}
$$

and $\left.\left.V^{\theta}\right|_{U\left(V^{\theta}, V^{\theta^{\prime}}\right)} \cong V^{\theta^{\prime}}\right|_{U\left(V^{\theta}, V^{\theta^{\prime}}\right)}$ by the conditions coming from the walls meeting the interior of the polytopes $\Theta\left(V^{\theta}(y)\right), \Theta\left(V^{\theta^{\prime}}(y)\right)$.

To study the conditions coming from walls that separate polytopes $\Theta(V(y))$ we consider the schemes of representations of $Q$ for weights in a GIT equivalence class $C_{\tau}$ of codimension one with adjacent chambers $C_{\theta}, C_{\theta^{\prime}}$. For $Q=Q_{n}, C_{\tau} \cap \Delta(2, n)$ is contained in a wall of the form $W_{\left\{J, J^{\complement}\right\}}$ for some $J \subset\{1, \ldots, n\}, 2 \leqslant|J| \leqslant n-2$. For $Q=P_{n}, C_{\tau} \cap \Delta^{1} \times \Delta^{n-1}$ is contained in a wall of the form $W_{J}$ for some $J \subset\{1, \ldots, n\}$, $1 \leqslant|J| \leqslant n-1$. In case $Q=Q_{n}$ let $i \in J^{\complement}, j \in J$, in case $Q=P_{n}$ let $i=0, j=\infty$. Let $Z_{\tau} \subset R^{\tau}(Q)$ be the closed subscheme of strictly $\tau$-semistable points and $V^{\tau}$ its tautological representation considered as tuple of sections $\left(s_{1}^{\tau}, \ldots, s_{n}^{\tau}\right)$ of $\mathbb{P}_{Z_{\tau}}^{1} \rightarrow Z_{\tau}$. The geometric fibres of $Z_{\tau}$ over $S$ consist of the following three strata: the subset where both conditions $s_{l}^{\tau}=s_{j}^{\tau} \Leftrightarrow l \in J$ and $s_{k}^{\tau}=s_{i}^{\tau} \Leftrightarrow k \in J^{\complement}$ are satisfied, and the two subsets $Z_{\tau, J}, Z_{\tau, J^{\mathrm{C}}}$ where only the first (resp. the second) condition is satisfied. In case $Q=Q_{n}$ assume $\sum_{j \in J} \theta_{j}>1$, then $\sum_{i \in J^{\mathrm{C}}} \theta_{i}^{\prime}>1$ and $Z_{\tau, J}=Z_{\tau} \cap R^{\theta^{\prime}}(Q)$, $Z_{\tau, J^{\mathrm{C}}}=Z_{\tau} \cap R^{\theta}(Q)$. The image of $Z_{\tau, J}$ forms a projective space $\mathbb{P}_{S}^{\left|J^{\mathrm{C}}\right|-2} \subset \mathcal{M}^{\theta^{\prime}}(Q)$ the image of $Z_{\tau, J^{c}}$ forms a projective space $\mathbb{P}_{S}^{|J|-2} \subset \mathcal{M}^{\theta}(Q)$ and the image of $Z_{\tau}$ forms a subscheme $Z^{\tau} \subset \mathcal{M}^{\tau}(Q)$ isomorphic to the base scheme $S$. The morphisms $\mathcal{M}^{\theta}(Q) \rightarrow \mathcal{M}^{\tau}(Q) \leftarrow \mathcal{M}^{\theta^{\prime}}(Q)$ restrict to morphisms $\mathbb{P}_{S}^{|J|-2} \rightarrow Z^{\tau} \leftarrow \mathbb{P}_{S}^{\left|J^{\complement}\right|-2}$ and are isomorphisms elsewhere. The case $Q=P_{n}$ is similar with projective spaces $\mathbb{P}_{S}^{|J|-1} \subset$ $\mathcal{M}^{\theta}(Q), \mathbb{P}_{S}^{\left|J^{\complement}\right|-1} \subset \mathcal{M}^{\theta^{\prime}}(Q)$.

Coordinate functions on $R^{*}(Q)$ are given by the tautological family on $R^{*}(Q)$, considered as sections $s=\left(s_{1}=\left(s_{1,0}: s_{1,1}\right), \ldots, s_{n}=\left(s_{n, 0}: s_{n, 1}\right)\right)$ of $\mathbb{P}_{R^{*}(Q)}^{1} \rightarrow$ $R^{*}(Q)$, as follows. In case $Q=Q_{n}$ for $i, j \in\{1, \ldots, n\}$ over the invariant open subscheme $\left\{y \mid s_{i}(y) \neq s_{j}(y)\right\} \subset R^{*}(Q)$ there is the section $\left(\begin{array}{cc}s_{i, 1} & -s_{i, 0} \\ -s_{j, 1} & s_{j, 0}\end{array}\right)$ in $\operatorname{PGL}(2)$ that transforms the tautological family $s$ to the family $\tilde{s}$ with $\tilde{s}_{i}=(0: 1), \tilde{s}_{j}=(1: 0)$. In case $Q=P_{n}$ we have the additional sections $s_{i}=s_{0}=(0: 1), s_{j}=s_{\infty}=(1: 0)$ and we set $\tilde{s}=s$. The invariant open subscheme

$$
U_{\tau}=\left\{y \mid s_{i}(y) \neq s_{j}(y), \forall k \in J^{\complement}: s_{k}(y) \neq s_{j}(y), \forall l \in J: s_{l}(y) \neq s_{i}(y)\right\} \subset R^{*}(Q)
$$

contains $Z_{\tau}$. The algebra of invariant functions on $U_{\tau}$ corresponds to the subalgebra of torus invariant functions in the $\mathcal{O}_{S}$-algebra generated by $\frac{\tilde{s}_{l, 1}}{\tilde{s}_{l, 0}}$ for $l \in J$ and $\frac{\tilde{s}_{k, 0}}{\tilde{s}_{k, 1}}$ for $k \in J^{\complement}$. This $\mathcal{O}_{S}$-algebra of torus invariants is generated by $f_{k, l}^{i, j}=\frac{\tilde{s}_{k, 0} \tilde{s}_{l, 1}}{\tilde{s}_{k, 1} \tilde{s}_{l, 0}}$ for $l \in J$, $k \in J^{\complement}$. The function $f_{k, l}^{i, j}$ can be written in terms of $s$ as

$$
f_{k, l}^{i, j}=\frac{\left(s_{j, 0} s_{l, 1}-s_{j, 1} s_{l, 0}\right)\left(s_{i, 1} s_{k, 0}-s_{i, 0} s_{k, 1}\right)}{\left(s_{i, 1} s_{l, 0}-s_{i, 0} s_{l, 1}\right)\left(s_{j, 0} s_{k, 1}-s_{j, 1} s_{k, 0}\right)} .
$$

These invariant functions are sometimes called the cross-ratios of four sections. Since invariant regular functions on the representation space correspond to regular functions on the moduli spaces we obtain the following result.

LEMMA 2.13.

(a) The invariant regular functions $f_{k, l}^{i, j}$ on $U_{\tau}$ define regular functions on the image of $U_{\tau} \cap R^{\tau}(Q)$ (resp. $U_{\tau} \cap R^{\theta}(Q), U_{\tau} \cap R^{\theta^{\prime}}(Q)$ ) in $\mathcal{M}^{\tau}(Q)$ (resp. $\left.\mathcal{M}^{\theta}(Q), \mathcal{M}^{\theta^{\prime}}(Q)\right)$ which we also denote $f_{k, l}^{i, j}$. These satisfy $\varphi_{\theta, \tau}^{*}\left(f_{k, l}^{i, j}\right)=f_{k, l}^{i, j}$, $\varphi_{\theta^{\prime}, \tau}^{*}\left(f_{k, l}^{i, j}\right)=f_{k, l}^{i, j}$. 
(b) For $z \in Z^{\tau}$ the local ring $\mathcal{O}_{\mathcal{M}^{\tau}(Q), z}$ is the localisation of an $\mathcal{O}_{S}$-algebra generated by the functions $f_{k, l}^{i, j}$ for $l \in J, k \in J^{\complement}$.

We express the conditions in (7) for a family $\left(V^{\theta}\right)_{\theta}$ locally in terms of equations.

Lemma 2.14. Let $V^{\theta}, V^{\theta^{\prime}}$ be free representations of $Q=Q_{n}$ or $Q=P_{n}$ over an $S$-scheme $Y$ stable with respect to generic $\theta, \theta^{\prime}$. Assume $V^{\theta}, V^{\theta^{\prime}}$ are part of a collection as in (7). We consider $V^{\theta}, V^{\theta^{\prime}}$ as tuples of sections $\left(s_{i}^{\theta}\right)_{i}=\left(s_{i, 0}^{\theta}: s_{i, 1}^{\theta}\right)_{i},\left(s_{i}^{\theta^{\prime}}\right)_{i}=\left(s_{i, 0}^{\theta^{\prime}}\right.$ : $\left.s_{i, 1}^{\theta^{\prime}}\right)_{i}$ of $\mathbb{P}_{Y}^{1}$, in case $Q=P_{n}$ we add the sections $s_{0}^{\theta}, s_{0}^{\theta^{\prime}}=(0: 1), s_{\infty}^{\theta}, s_{\infty}^{\theta^{\prime}}=(1: 0)$. In case $Q=Q_{n}$ let $i, j \in\{1, \ldots, n\}$, in case $Q=P_{n}$ let $i=0, j=\infty$. Let $U_{i, j}=$ $\left\{y \mid s_{i}^{\theta}(y) \neq s_{j}^{\theta}(y), s_{i}^{\theta^{\prime}}(y) \neq s_{j}^{\theta^{\prime}}(y)\right\} \subseteq Y$. We choose homogeneous coordinates $x_{0}^{\theta}, x_{1}^{\theta}$ and $x_{0}^{\theta^{\prime}}, x_{1}^{\theta^{\prime}}$ of $\mathbb{P}_{U_{i, j}}^{1}$ such that $s_{i}^{\theta}=(0: 1), s_{j}^{\theta}=(1: 0)$ with respect to $x_{0}^{\theta}, x_{1}^{\theta}$ and $s_{i}^{\theta^{\prime}}=(0: 1), s_{j}^{\theta^{\prime}}=(1: 0)$ with respect to $x_{0}^{\theta^{\prime}}, x_{1}^{\theta^{\prime}}$. Then $V^{\theta}$ and $V^{\theta^{\prime}}$ are over $U_{i, j}$ related by the equations

$$
s_{k, 0}^{\theta} s_{l, 1}^{\theta} s_{k, 1}^{\theta^{\prime}} s_{l, 0}^{\theta^{\prime}}=s_{k, 1}^{\theta} s_{l, 0}^{\theta} s_{k, 0}^{\theta^{\prime}} s_{l, 1}^{\theta^{\prime}}
$$

for all $k, l \in\{1, \ldots, n\}$.

Proof. Over $U\left(V^{\theta}, V^{\theta^{\prime}}\right)$ the equations hold because $\left.\left.V^{\theta}\right|_{U\left(V^{\theta}, V^{\theta^{\prime}}\right)} \cong V^{\theta^{\prime}}\right|_{U\left(V^{\theta}, V^{\theta^{\prime}}\right)}$.

Consider the local situation around a point $y \in U_{i, j} \backslash U\left(V^{\theta}, V^{\theta^{\prime}}\right)$. Assume first that the polytopes $\Theta\left(V^{\theta}(y)\right)$ and $\Theta\left(V^{\theta^{\prime}}(y)\right)$ meet in an inner wall $W$. In case $Q=P_{n}$ let $W=W_{J}$ and we can assume that $s_{h}^{\theta}(y)=s_{j}^{\theta}(y)=s_{\infty}^{\theta}(y)=(1: 0)$ for $h \in J$ and $s_{h}^{\theta^{\prime}}(y)=s_{i}^{\theta^{\prime}}(y)=s_{0}^{\theta^{\prime}}(y)=(0: 1)$ for $h \in J^{\complement}$. In case $Q=Q_{n}$ let $W=W_{\left\{J, J^{\mathrm{C}}\right\}}$ and we can assume $j \in J, i \in J^{\complement}$ and $s_{h}^{\theta}(y)=s_{j}^{\theta}(y)=(1: 0)$ for $h \in J, s_{h}^{\theta^{\prime}}(y)=$ $s_{i}^{\theta^{\prime}}(y)=(0: 1)$ for $h \in J^{\complement}$. For $k, l$ there are the cases: $k, l \in J$ (similar: $k, l \in J^{\complement}$ ) or $k \in J, l \in J^{\complement}$ (similar: $k \in J^{\complement}, l \in J$ ).

Case $k \in J^{\complement}, l \in J$ : We have $s_{l, 0}^{\theta}(y), s_{l, 0}^{\theta^{\prime}}(y) \neq 0$ and $s_{k, 1}^{\theta}(y), s_{k, 1}^{\theta^{\prime}}(y) \neq 0$. In a neighbourhood of $y$ equation (8) is equivalent to the equation $f_{k, l}^{\theta, i, j}=f_{k, l}^{\theta^{\prime}, i, j}$ with $f_{k, l}^{\theta, i, j}=\frac{s_{k, 0}^{\theta} s_{l, 1}^{\theta}}{s_{k, 1}^{\theta} s_{l, 0}^{\theta}}, f_{k, l}^{\theta^{\prime}, i, j}=\frac{s_{k, 0}^{\theta^{\prime}} s_{l, 1}^{\theta^{\prime}}}{s_{k, 1}^{\theta^{\prime}} s_{l, 0}^{\theta^{\prime}}}$. The regular functions $f_{k, l}^{\theta, i, j}$ (resp. $f_{k, l}^{\theta^{\prime}, i, j}$ ) are pullbacks of the regular functions $f_{k, l}^{i, j}$ on $\mathcal{M}^{\theta}(Q)$ (resp. $\left.\mathcal{M}^{\theta^{\prime}}(Q)\right)$ via $\varphi_{V^{\theta}}$ (resp. $\varphi_{V^{\theta^{\prime}}}$ ). Because $\varphi_{\theta, \tau} \circ \varphi_{V^{\theta}}=\varphi_{\theta^{\prime}, \tau} \circ \varphi_{V^{\theta^{\prime}}}$ and using lemma 2.13.(a) it follows $f_{k, l}^{\theta, i, j}=f_{k, l}^{\theta^{\prime}, i, j}$.

Case $k, l \in J$ : We have $s_{k, 0}^{\theta}(y), s_{k, 0}^{\theta^{\prime}}(y) \neq 0$ and $s_{l, 0}^{\theta}(y), s_{l, 0}^{\theta^{\prime}}(y) \neq 0$. In a neighbourhood of $y$ equation (8) is equivalent to the equation $\frac{s_{l, 1}^{\theta}}{s_{l, 0}^{\theta}} \frac{\theta_{k, 1}^{\theta^{\prime}}}{s_{k, 0}^{\theta_{1}^{\prime}}}=\frac{s_{k, 1}^{\theta}}{s_{k, 0}^{\theta}} \frac{\theta_{l, 1}^{\theta^{\prime}}}{s_{l, 0}^{\prime}}$. Multiplying with $\frac{s_{g, 0}^{\theta} s_{h, 0}^{\theta^{\prime}}}{s_{g, 1}^{\theta} s_{h, 1}^{\theta^{\prime}}}$ for $g \in J^{\complement}$ such that $s_{g}^{\theta}(y) \neq(0: 1)$ and $h \in J$ such that $s_{h}^{\theta^{\prime}}(y) \neq(1: 0)$, we obtain the equivalent equation $f_{g, l}^{\theta, i, j} f_{h, k}^{\theta^{\prime}, i, j}=f_{g, k}^{\theta, i, j} f_{h, l}^{\theta^{\prime}, i, j}$. This equation holds because by the first case we have $f_{g, l}^{\theta, i, j}=f_{g, l}^{\theta^{\prime}, i, j}, f_{g, k}^{\theta, i, j}=f_{g, k}^{\theta^{\prime}, i, j}$.

This shows that equations (8) hold for $V^{\theta}, V^{\theta^{\prime}}$ in a neighbourhood of $y$ if the polytopes $\Theta\left(V^{\theta}(y)\right)$ and $\Theta\left(V^{\theta^{\prime}}(y)\right)$ have overlapping interiors or meet in an inner wall.

To show the general case we show that if the union of the sets of walls separating $\Theta\left(V^{\theta}(y)\right), \Theta\left(V^{\theta^{\prime}}(y)\right)$ and $\Theta\left(V^{\theta^{\prime}}(y)\right), \Theta\left(V^{\theta^{\prime \prime}}(y)\right)$ are exactly the walls that separate $\Theta\left(V^{\theta}(y)\right), \Theta\left(V^{\theta^{\prime \prime}}(y)\right)$ and if $V^{\theta}, V^{\theta^{\prime}}$ and $V^{\theta^{\prime}}, V^{\theta^{\prime \prime}}$ in a neighbourhood of $y$ are related by equations of the form (8), then so are $V^{\theta}, V^{\theta^{\prime \prime}}$. Assume that $\Theta\left(V^{\theta}(y)\right)$ and $\Theta\left(V^{\theta^{\prime}}(y)\right)$ are separated by an inner wall $W_{J}$ (resp. $\left.W_{\left\{J, J^{\mathrm{C}}\right\}}\right)$ and that $\Theta\left(V^{\theta^{\prime}}(y)\right)$ and $\Theta\left(V^{\theta^{\prime \prime}}(y)\right)$ are separated by an inner wall $W_{J^{\prime}}\left(\right.$ resp. $\left.W_{\left\{J^{\prime},\left(J^{\prime}\right)^{\complement}\right\}}\right)$ such that $J \subset J^{\prime}$, 
these walls are part of the boundary of $\Theta\left(V^{\theta^{\prime}}(y)\right)$ and both walls separate $\Theta\left(V^{\theta}(y)\right)$ and $\Theta\left(V^{\theta^{\prime \prime}}(y)\right)$. If $s_{i}^{\theta}(y) \neq s_{j}^{\theta}(y), s_{i}^{\theta^{\prime \prime}}(y) \neq s_{j}^{\theta^{\prime \prime}}(y)$ then either $i \in J, j \in\left(J^{\prime}\right)^{\complement}$ or $j \in J$, $i \in\left(J^{\prime}\right)^{\complement}$ and thus $s_{i}^{\theta^{\prime}}(y) \neq s_{j}^{\theta^{\prime}}(y)$. We assume $j \in J, i \in\left(J^{\prime}\right)^{\complement}$.

Case $k \in J^{\prime}, l \in J^{\complement}$ (similar: $\left.k \in\left(J^{\prime}\right)^{\complement}, l \in J\right)$. It is $s_{k}^{\theta^{\prime}}(y) \neq(0: 1), s_{l}^{\theta^{\prime}}(y) \neq(1: 0)$ and in a neighbourhood of $y$ we have

$$
s_{k, 0}^{\theta} s_{l, 1}^{\theta} s_{k, 1}^{\theta^{\prime \prime}} s_{l, 0}^{\theta^{\prime \prime}} s_{k, 0}^{\theta^{\prime}} s_{l, 1}^{\theta^{\prime}}=s_{k, 0}^{\theta} s_{l, 1}^{\theta} s_{k, 1}^{\theta^{\prime}} s_{l, 0}^{\theta^{\prime}} s_{k, 0}^{\theta^{\prime \prime}} s_{l, 1}^{\theta^{\prime \prime}}=s_{k, 0}^{\theta^{\prime}} s_{l, 1}^{\theta^{\prime}} s_{k, 1}^{\theta} s_{l, 0}^{\theta} s_{k, 0}^{\theta^{\prime \prime}} s_{l, 1}^{\theta^{\prime \prime}},
$$

thus $s_{k, 0}^{\theta} s_{l, 1}^{\theta} s_{k, 1}^{\theta^{\prime \prime}} s_{l, 0}^{\theta^{\prime \prime}}=s_{k, 1}^{\theta} s_{l, 0}^{\theta} s_{k, 0}^{\theta^{\prime \prime}} s_{l, 1}^{\theta^{\prime \prime}}$.

Case $k \in J^{\prime}, l \in J$ (similar: $\left.k \in\left(J^{\prime}\right)^{\complement}, l \in J^{\complement}\right)$. Choose $h \in J^{\complement} \cap J^{\prime}$. Then $s_{k}^{\theta^{\prime}}(y), s_{l}^{\theta^{\prime}}(y) \neq(0: 1)$ and $s_{h}^{\theta}(y)=(0: 1), s_{h}^{\theta^{\prime \prime}}(y)=(1: 0), s_{h}^{\theta^{\prime}}(y) \neq(1: 0),(0: 1)$. In a neighbourhood of $y$ we have

$$
\begin{aligned}
s_{k, 0}^{\theta} s_{l, 1}^{\theta} s_{k, 1}^{\theta^{\prime \prime}} s_{l, 0}^{\theta^{\prime \prime}} s_{k, 0}^{\theta^{\prime}} s_{l, 0}^{\theta^{\prime}} s_{h, 1}^{\theta^{\prime}} s_{h, 1}^{\theta} s_{h, 0}^{\theta^{\prime \prime}} & =s_{k, 0}^{\theta} s_{l, 1}^{\theta} s_{k, 1}^{\theta^{\prime}} s_{l, 0}^{\theta^{\prime \prime}} s_{k, 0}^{\theta^{\prime \prime}} s_{l, 0}^{\theta^{\prime}} s_{h, 1}^{\theta^{\prime \prime}} s_{h, 1}^{\theta} s_{h, 0}^{\theta^{\prime}} \\
& =s_{k, 0}^{\theta^{\prime}} s_{l, 1}^{\theta} s_{k, 1}^{\theta} s_{l, 0}^{\theta^{\prime \prime}} s_{k, 0}^{\theta^{\prime \prime}} s_{l, 0}^{\theta^{\prime}} s_{h, 1}^{\theta^{\prime \prime}} s_{h, 1}^{\theta^{\prime}} s_{h, 0}^{\theta} \\
& =s_{k, 0}^{\theta^{\prime}} s_{l, 1}^{\theta^{\prime}} s_{k, 1}^{\theta} s_{l, 0}^{\theta^{\prime \prime}} s_{k, 0}^{\theta^{\prime \prime}} s_{l, 0}^{\theta} s_{h, 1}^{\theta^{\prime \prime}} s_{h, 1}^{\theta} s_{h, 0}^{\theta^{\prime}} \\
& =s_{k, 0}^{\theta^{\prime}} s_{l, 1}^{\theta^{\prime \prime}} s_{k, 1}^{\theta} s_{l, 0}^{\theta^{\prime}} s_{k, 0}^{\theta^{\prime \prime}} s_{l, 0}^{\theta} s_{h, 1}^{\theta^{\prime}} s_{h, 1}^{\theta} s_{h, 0}^{\theta^{\prime \prime}}
\end{aligned}
$$

$\operatorname{thus} s_{k, 0}^{\theta} s_{l, 1}^{\theta} s_{k, 1}^{\theta^{\prime \prime}} s_{l, 0}^{\theta^{\prime \prime}}=s_{k, 1}^{\theta} s_{l, 0}^{\theta} s_{k, 0}^{\theta^{\prime \prime}} s_{l, 1}^{\theta^{\prime \prime}}$.

Proposition 2.15. For $Q=Q_{n}$ or $Q=P_{n}$ the functor of the inverse limit of moduli spaces of representations is isomorphic to the functor $\operatorname{Lim}(Q)$ defined by

$$
Y \mapsto\left\{\left(\varphi_{V^{\theta}}\right)_{\theta} \in \prod_{\theta} \mathcal{M}_{\text {generic }}^{\theta}(Q)(Y) \mid \text { locally equations (8) hold for }\left(V^{\theta}\right)_{\theta}\right\}
$$

where the product is over representatives $\theta$ of the generic GIT equivalence classes and $\varphi_{V^{\theta}}: Y \rightarrow \mathcal{M}^{\theta}(Q)$ is the morphism determined by a $\theta$-stable representation $V^{\theta}$ over $Y$.

Proof. By lemma 2.14 for a family $\left(V^{\theta}\right)_{\theta}$ satisfying the conditions of (7) the equations (8) hold locally. We show the opposite implication.

Let $\left(V^{\theta}\right)_{\theta}$ be a family of representations over an $S$-scheme $Y$ as in (9). Let $y \in Y$. Let $C_{\tau}$ be an equivalence class of codimension 1 in the interior and $\theta, \theta^{\prime}$ generic such that $\bar{C}_{\tau}=\bar{C}_{\theta} \cap \bar{C}_{\theta^{\prime}}$. We can consider $V^{\theta}, V^{\theta^{\prime}}$ in a neighbourhood $U$ of $y$ as tuples of sections $\left(s_{i}^{\theta}\right)_{i},\left(s_{i}^{\theta^{\prime}}\right)_{i}$ of $\mathbb{P}_{U}^{1} \rightarrow U$. In case $Q=Q_{n}$ let $i, j \in\{1, \ldots, n\}$ such that $s_{i}^{\theta}(y) \neq s_{j}^{\theta}(y)$ and $s_{i}^{\theta^{\prime}}(y) \neq s_{j}^{\theta^{\prime}}(y)$ and choose coordinates such that $s_{i}^{\theta}, s_{i}^{\theta^{\prime}}=(0: 1)$ and $s_{j}^{\theta}, s_{j}^{\theta^{\prime}}=(1: 0)$. In case $Q=P_{n}$ let $i=0, j=\infty$, then $s_{i}^{\theta}=s_{0}^{\theta}=(0: 1)$, $s_{j}^{\theta}=s_{\infty}^{\theta}=(1: 0)$ and the same for $\theta^{\prime}$.

If there exists $k \in\{1, \ldots, n\}$ such that $s_{k}^{\theta}(y) \neq(0: 1),(1: 0), s_{k}^{\theta^{\prime}}(y) \neq(0: 1),(1: 0)$ then the equations $s_{k, 0}^{\theta} s_{l, 1}^{\theta} s_{k, 1}^{\theta^{\prime}} s_{l, 0}^{\theta^{\prime}}=s_{k, 1}^{\theta} s_{l, 0}^{\theta} s_{k, 0}^{\theta^{\prime}} s_{l, 1}^{\theta^{\prime}}$ show that in a neighbourhood of $y V^{\theta} \cong V^{\theta^{\prime}}$ and thus $\varphi_{\theta, \tau} \circ \varphi_{V^{\theta}}=\varphi_{\theta^{\prime}, \tau} \circ \varphi_{V^{\theta^{\prime}}}$.

Otherwise there is $J \subset\{1, \ldots, n\}$, where we can assume that $j \in J, i \in J^{\complement}$, such that $\forall k \in J^{\complement}: s_{k}^{\theta}(y)=(0: 1), s_{k}^{\theta^{\prime}}(y) \neq(1: 0)$ and $\forall l \in J: s_{l}^{\theta}(y) \neq(0: 1)$, $s_{l}^{\theta^{\prime}}(y)=(1: 0)$. Let $x \in S$ be the image of $y \in Y$ and $z \in Z^{\tau}$ the point over $x$, then $\varphi_{\theta, \tau} \circ \varphi_{V^{\theta}}(y)=z=\varphi_{\theta^{\prime}, \tau} \circ \varphi_{V^{\theta^{\prime}}}(y)$. As equations (8) hold, the functions $f_{k, l}^{\theta, i, j}=\frac{s_{k, 0}^{\theta} s_{l, 1}^{\theta}}{s_{k, 1}^{\theta} s_{l, 0}^{\theta}}$ and $f_{k, l}^{\theta^{\prime}, i, j}=\frac{s_{k, s^{\prime}}^{\theta^{\prime}} \theta_{l, 1}^{\theta^{\prime}}}{s_{k, 1}^{\theta^{\prime}} s_{l, 0}^{\theta^{\prime}}}$ coincide. We may consider these functions as elements of the local ring $\mathcal{O}_{Y, y}$. Using lemma 2.13 , these are the pullbacks of the functions $f_{k, l}^{i, j}$ in $\mathcal{O}_{\mathcal{M}^{\tau}(Q), z}$ 
under $\varphi_{\theta, \tau} \circ \varphi_{V^{\theta}}$ and $\varphi_{\theta^{\prime}, \tau} \circ \varphi_{V^{\theta^{\prime}}}$, and it follows that the homomorphisms of local rings $\mathcal{O}_{\mathcal{M}^{\tau}(Q), z} \rightarrow \mathcal{O}_{Y, y}$ agree.

Since $\varphi_{\theta, \tau} \circ \varphi_{V^{\theta}}$ and $\varphi_{\theta^{\prime}, \tau} \circ \varphi_{V^{\theta^{\prime}}}$ coincide as maps of sets of points and in each point the induced homomorphisms of local rings coincide, it follows $\varphi_{\theta, \tau} \circ \varphi_{V^{\theta}}=$ $\varphi_{\theta^{\prime}, \tau} \circ \varphi_{V^{\theta^{\prime}}}$.

Let $\left(V^{\theta}\right)_{\theta}$ be a family of representations over $Y$ satisfying the conditions of (7) or equivalently (9). For $V^{\theta}, V^{\theta^{\prime}}$ we have the natural structure of a closed subscheme $Z\left(V^{\theta}, V^{\theta^{\prime}}\right) \subseteq Y$ supported on the closed subset $Y \backslash U\left(V^{\theta}, V^{\theta^{\prime}}\right)$. Under the assumptions and notations of lemma 2.14 let $y \in U_{i, j}$ and assume $k, l \in\{1, \ldots, n\}$ such that $s_{k}^{\theta} \neq(0: 1),(1: 0), s_{k}^{\theta^{\prime}} \neq(0: 1)$ and $s_{l}^{\theta^{\prime}} \neq(0: 1),(1: 0), s_{l}^{\theta} \neq(1: 0)$. Because equations (8) hold in a neighbourhood of $y$, the scheme defined by the equation $s_{k}^{\theta^{\prime}}=(1: 0)$ coincides with the scheme defined by the equation $s_{l}^{\theta}=(0: 1)$ and does not depend on the choice of $k, l$. The scheme $Z\left(V^{\theta}, V^{\theta^{\prime}}\right)$ can be defined in a neighbourhood of $y$ by each of these equations.

The following proposition gives a geometric interpretation of the condition in (9) that two representations are related by equations (8), and indicates the relation between inverse limits of moduli spaces of representations for the quivers $P_{n}, Q_{n}$ and moduli spaces of chains and trees of $\mathbb{P}^{1}$ 's with marked points.

Proposition 2.16. Let $V^{\theta}, V^{\theta^{\prime}}$ be free representations of $Q=Q_{n}$ or $Q=P_{n}$ over an $S$-scheme $Y$ stable with respect to generic $\theta, \theta^{\prime}$. We use the notations of lemma 2.14 and assume that $V^{\theta}, V^{\theta^{\prime}}$ over $U_{i, j}$ are related by equations (8). Consider over $U_{i, j}$ the equations

$$
s_{k, 0}^{\theta} s_{k, 1}^{\theta^{\prime}} x_{1}^{\theta} x_{0}^{\theta^{\prime}}=s_{k, 1}^{\theta} s_{k, 0}^{\theta^{\prime}} x_{0}^{\theta} x_{1}^{\theta^{\prime}}
$$

(a) Let $C_{i, j} \subset\left(\mathbb{P}^{1}\right)_{U_{i, j}}^{2}$ be the closed subscheme defined by the equations (10) for all $k$. For given $k$ let

$U_{i, j, k}=\left\{y \in U_{i, j} \mid s_{i}^{\theta}(y), s_{j}^{\theta}(y), s_{k}^{\theta}(y)\right.$ distinct or $s_{i}^{\theta^{\prime}}(y), s_{j}^{\theta^{\prime}}(y), s_{k}^{\theta^{\prime}}(y)$ distinct $\}$.

Then over $U_{i, j, k}$ the subscheme $C_{i, j, k}=C_{i, j} \times_{U_{i, j}} U_{i, j, k} \subset\left(\mathbb{P}^{1}\right)_{U_{i, j, k}}^{2}$ is given by the single equation (10) for this $k$.

(b) The curves $C_{i, j} \subset\left(\mathbb{P}^{1}\right)_{U_{i, j}}^{2}$ glue (with the appropriate coordinate changes) to a reduced curve $C \subset\left(\mathbb{P}^{1}\right)_{Y}^{2}$ flat over $Y$, which contains all pairs of sections $\left(s_{l}^{\theta}, s_{l}^{\theta^{\prime}}\right)$, is isomorphic to $\mathbb{P}_{U\left(V^{\theta}, V^{\theta^{\prime}}\right)}^{1}$ over $U\left(V^{\theta}, V^{\theta^{\prime}}\right) \subseteq Y$ via its two projections, and degenerates exactly over the subscheme $Z\left(V^{\theta}, V^{\theta^{\prime}}\right) \subseteq Y$ to a chain of two $\mathbb{P}^{1}$ 's intersecting transversally such that each projection defines an isomorphism on one component and contracts the other to a reduced point.

(c) The curve $C$ induces morphisms $\mathbb{P}_{Y}^{1} \leftrightarrows \mathbb{P}_{Y}^{1}$ which degenerate to $\mathbb{P}^{1} \rightarrow$ pt. over $Z\left(V^{\theta}, V^{\theta^{\prime}}\right)$ and which restrict to mutually inverse isomorphisms $\mathbb{P}_{U\left(V^{\theta}, V^{\theta^{\prime}}\right)}^{1} \leftrightarrow \mathbb{P}_{U\left(V^{\theta}, V^{\theta^{\prime}}\right)}^{1}$ that give rise to the isomorphism $\left.V^{\theta}\right|_{U\left(V^{\theta}, V^{\theta^{\prime}}\right)} \cong$ $\left.V^{\theta^{\prime}}\right|_{U\left(V^{\theta}, V^{\theta^{\prime}}\right)}$.

Proof. (a) Assume that $s_{k}^{\theta}(y) \neq(0: 1),(1: 0)$. We can further assume $s_{k}^{\theta^{\prime}}(y) \neq(1: 0)$ (similar: $\left.s_{k}^{\theta^{\prime}}(y) \neq(0: 1)\right)$. Then in a neighbourhood of $y$ for all $l$ we have

$$
s_{l, 0}^{\theta} s_{l, 1}^{\theta^{\prime}} x_{1}^{\theta} x_{0}^{\theta^{\prime}}=s_{l, 0}^{\theta} s_{l, 1}^{\theta^{\prime}} \frac{s_{k, 1}^{\theta} s_{k, 0}^{\theta^{\prime}}}{s_{k, 0}^{\theta} s_{k, 1}^{\theta^{\prime}}} x_{0}^{\theta} x_{1}^{\theta^{\prime}}=s_{l, 1}^{\theta} s_{l, 0}^{\theta^{\prime}} x_{0}^{\theta} x_{1}^{\theta^{\prime}}
$$

using equation (10) for $k$ and equation (8) for $k, l$. 
(b) To show that the curves $C_{i, j}$ glue, it suffices to show that the curves $C_{i, j}$ and $C_{i, j^{\prime}}$ (and similar: $C_{i, j}$ and $C_{i^{\prime}, j}$ ) coincide over $U_{i, j} \cap U_{i . j^{\prime}}$ after the base changes

$$
\left(\begin{array}{c}
s_{k, 0}^{\theta, i, j^{\prime}} \\
s_{k, i}^{\theta, i, j^{\prime}}
\end{array}\right)=\left(\begin{array}{cc}
s_{j, 0}^{\theta, i, j^{\prime}} & 0 \\
\theta, i, j^{\prime} & a
\end{array}\right)\left(\begin{array}{c}
s_{k, 0}^{\theta, i, j} \\
s_{j, 1}^{\theta, i, j} \\
s_{k, 1}
\end{array}\right) \quad \text { and } \quad\left(\begin{array}{c}
s_{k, 0}^{\theta, i, j} \\
s_{k, i, j}^{\theta, i, j}
\end{array}\right)=\left(\begin{array}{cc}
a & 0 \\
-s_{j, 1}^{\theta, i, j^{\prime}} & s_{j, 0}^{\theta, i, j^{\prime}}
\end{array}\right)\left(\begin{array}{c}
s_{k, 0}^{\theta, i, j^{\prime}} \\
s_{k, i}^{\theta, i, j^{\prime}}
\end{array}\right),
$$

where $a$ satisfies the relation $s_{j^{\prime}, 0}^{\theta, i, j} s_{j, 1}^{\theta, i, j^{\prime}}+a s_{j^{\prime}, 1}^{\theta, i, j}=0$ and $s_{k}^{\theta}=\left(s_{k, 0}^{\theta, i, j}: s_{k, 1}^{\theta, i, j}\right)$ with respect to coordinates $x_{0}^{\theta, i, j}, x_{1}^{\theta, i, j}$ such that $\left(s_{i, 0}^{\theta, i, j}: s_{i, 1}^{\theta, i, j}\right)=(0: 1),\left(s_{j, 0}^{\theta, i, j}: s_{j, 1}^{\theta, i, j}\right)=$ $(1: 0)$ (similar for $\left.\theta^{\prime}, j^{\prime}\right)$. Applying these base changes one verifies that the equations (10) hold for all $k$ with respect to $i, j$ if and only if they hold for all $k$ with respect to $i, j^{\prime}$. The properties of $C$ follow from the properties of $C_{i, j, k}$ given by the single equation (10) for $k$, which are easy to verify.

(c) follows from (b).

REMARK 2.17. Proposition 2.16 allows to restrict the sets of equations required to hold in functors like (9) in proposition 2.15. All equations (8) relating $V^{\theta}, V^{\theta^{\prime}}$ hold if the equations with respect to choices of $i, j$ hold such that the corresponding sets $U_{i, j}$ cover $Y$. Also, with respect to fixed $i, j$, all equations relating $V^{\theta}, V^{\theta^{\prime}}$ hold over $U_{i, j}$ if those for certain $k$ hold over $U_{i, j, k}$ and these sets cover $U_{i, j}$.

\section{Losev-Manin and Grothendieck-Knudsen moduli Spaces and ROOT SYSTEMS OF TYPE $A$}

3.1. Losev-Manin and GrothendiecK-Knudsen moduli spaces. The LosevManin moduli spaces of stable $n$-pointed chains of $\mathbb{P}^{1}$ 's were introduced in [23].

Definition 3.1. A stable n-pointed chain of $\mathbb{P}^{1}$ over an algebraically closed field is a tuple $\left(C, s_{0}, s_{\infty}, s_{1}, \ldots, s_{n}\right)$, where $\left(C, s_{0}, s_{\infty}\right)$ is a chain of $\mathbb{P}^{1}$, i.e. its irreducible components are isomorphic to projective lines with two distinct closed points $\left(\mathbb{P}^{1}, 0, \infty\right)$ which intersect transversally such that the point 0 of one component meets the point $\infty$ of another component and the remaining points $0, \infty$ are denoted $s_{0}, s_{\infty}$, further $s_{1}, \ldots, s_{n} \in C$ are closed regular points different from $s_{0}, s_{\infty}$, and each component contains at least one of the marked points $s_{1}, \ldots, s_{n}$.

DeFinition - THEOREM 3.2 ([23]). Let $n \in \mathbb{Z}_{\geqslant 1}$. The Losev-Manin moduli space $\bar{L}_{n}$ is the fine moduli space of stable $n$-pointed chains of $\mathbb{P}^{1}$, i.e. $\bar{L}_{n}$ represents the moduli functor (denoted by the same symbol)

$$
Y \mapsto\left\{\text { isomorphism classes of stable } n \text {-pointed chains of } \mathbb{P}^{1} \text { over } Y\right\}
$$

where a stable n-pointed chain of $\mathbb{P}^{1}$ over a scheme $Y$ is a flat proper morphism $C \rightarrow Y$ with sections $s_{0}, s_{\infty}, s_{1}, \ldots, s_{n}: Y \rightarrow C$ such that the geometric fibres $\left(C_{y}, s_{0}(y), s_{\infty}(y), s_{1}(y), \ldots, s_{n}(y)\right)$ are stable $n$-pointed chains of $\mathbb{P}^{1}$ over algebraically closed fields.

$\bar{L}_{n}$ is toric, it compactifies the algebraic torus $L_{n}=\left(\mathbb{G}_{m}\right)^{n} / \mathbb{G}_{m}$, the moduli space of $n$ points in $\mathbb{P}^{1} \backslash\{0, \infty\}$. It has been shown in [23] that the moduli functor of stable $n$-pointed chains of $\mathbb{P}^{1}$ is represented by a projective scheme of relative dimension $n-1$, using an inductive construction of $\bar{L}_{n}$ together with the universal curve which is isomorphic to $\bar{L}_{n+1} \rightarrow \bar{L}_{n}$. This morphism $\bar{L}_{n+1} \rightarrow \bar{L}_{n}$, studied in a similar setting in [21], is a special case of the following morphisms that arise by forgetting sets of sections, see [3, Construction 3.15]. 
Proposition 3.3. Let $\varnothing \neq I \subseteq\{1, \ldots, n\}$. We write $\bar{L}_{I}$ for $\bar{L}_{|I|}$ with sections indexed by $I$. Then there is a morphism

$$
\gamma_{I}: \bar{L}_{n} \rightarrow \bar{L}_{I}
$$

such that a stable n-pointed chain is transformed to a stable I-pointed chain by forgetting the sections $\left\{s_{i} \mid i \notin I\right\}$ and contracting components which have become unstable.

The Grothendieck-Knudsen moduli space $\bar{M}_{0, n}$ is the moduli space of stable $n$ pointed curves of genus 0 , i.e. of stable $n$-pointed trees of $\mathbb{P}^{1}$. More generally, stable $n$-pointed curves of genus $g$ occur already in $[14$, I.5] and their moduli spaces and stacks have been systematically studied in [21].

Definition 3.4. A stable n-pointed curve of genus 0 over an algebraically closed field is a tuple $\left(C, s_{1}, \ldots, s_{n}\right)$ where $C$ is a complete connected reduced curve $C$ of genus 0 with at most ordinary double points, i.e. a tree of $\mathbb{P}^{1}, s_{1}, \ldots, s_{n}$ are closed points of $C$ such that each $s_{i}$ is a regular point of $C, s_{i} \neq s_{j}$ for $i \neq j$ and each component of $C$ has a least 3 special points (i.e. singular points and marked points $s_{i}$ ).

Definition - Theorem 3.5 ([21]). Let $n \in \mathbb{Z}_{\geqslant 3}$. The Grothendieck-Knudsen moduli space $\bar{M}_{0, n}$ is the fine moduli space of stable $n$-pointed curves of genus 0 , i.e. $\bar{M}_{0, n}$ represents the moduli functor (denoted by the same symbol)

$$
Y \mapsto\{\text { isomorphism classes of stable n-pointed curves of genus } 0 \text { over } Y\}
$$

where a stable n-pointed curve of genus 0 over a scheme $Y$ is a flat proper morphism $C \rightarrow Y$ with sections $s_{1}, \ldots, s_{n}: Y \rightarrow C$ such that the geometric fibres $\left(C_{y}, s_{1}(y), \ldots, s_{n}(y)\right)$ are stable $n$-pointed curves of genus 0 .

$\bar{M}_{0, n}$ compactifies the moduli space $M_{0, n}$ of $n$ distinct points in $\mathbb{P}^{1}$. The fact that the moduli functor of stable $n$-pointed curves of genus 0 is represented by a projective scheme of relative dimension $n-3$ has been shown in [21] using an inductive argument on $n$, showing that the universal family over $\bar{M}_{0, n}$ is formed by the morphism $\bar{M}_{0, n+1} \rightarrow \bar{M}_{0, n}$. This morphism is a special case of the following morphisms for inclusions $I \subset\{1, \ldots, n\}$ which can be defined by mapping a stable $n$-pointed tree $\left(C \rightarrow Y, s_{1}, \ldots, s_{n}\right)$ to its image under the morphism defined by the sheaf $\omega_{C / Y}\left(\sum_{i \in I} s_{i}\right)$, where $\omega_{C / Y}$ is the relative dualising sheaf (see [21]).

Proposition 3.6. Let $I \subseteq\{1, \ldots, n\},|I| \geqslant 3$. We write $\bar{M}_{0, I}$ for $\bar{M}_{0,|I|}$ with the sections of poined trees indexed by $I$. Then there is a morphism

$$
\gamma_{I}: \bar{M}_{0, n} \rightarrow \bar{M}_{0, I}
$$

such that a stable n-pointed tree is transformed to a stable I-pointed tree by forgetting the sections $\left\{s_{i} \mid i \notin I\right\}$ and contracting components which have become unstable.

3.2. Embeddings into PRoducts of $\mathbb{P}^{1}$ AND RELATion to RoOT SYSTEMS of TYPE $A$. We consider the cross-ratio varieties for root subsystems of type $A_{3}$ in $A_{n-1}$ defined and studied in $[27,28]$. It was already observed in [28] that the crossratio variety for root subsystems of type $A_{3}$ in $A_{n-1}$ should be isomorphic to the Grothendieck-Knudsen moduli space $\bar{M}_{0, n}$.

The root lattice of $A_{n}$ is the sublattice $M\left(A_{n-1}\right) \subset L\left(A_{n-1}\right)=\mathbb{Z}^{n}$ generated by the roots $\alpha_{i, j}=e_{i}-e_{j}$ where $i, j \in\{1, \ldots, n\}$ and $e_{1}, \ldots, e_{n}$ are the standard basis vectors of $\mathbb{Z}^{n}$. We have the lattice $L\left(A_{n-1}\right)^{*}$ dual to $L\left(A_{n-1}\right)$ and the lattice $N\left(A_{n-1}\right)=L\left(A_{n-1}\right)^{*} /(1, \ldots, 1) \mathbb{Z}$ dual to $M\left(A_{n-1}\right)$.

We consider cross-ratio varieties for root systems of type $A_{3}$ in $A_{n-1}$. Let $\mathbb{A}\left(L\left(A_{n-1}\right)\right)$ be the $n$-dimensional affine space with coordinates $t_{1}, \ldots, t_{n}$ corresponding to the basis $e_{1}, \ldots, e_{n}$. We have the open subscheme $U\left(A_{n-1}\right)=$ 
$\mathbb{A}\left(L\left(A_{n-1}\right)\right) \backslash \bigcup_{i \neq j}\left\{t_{i}=t_{j}\right\}$. For root subsystems $A_{3} \cong \Delta \subseteq A_{n-1}$ consisting of roots $\alpha_{i, j}$ for $i, j \in I=\left\{i_{1}, \ldots, i_{4}\right\} \subseteq\{1, \ldots, n\},|I|=4$ there is the morphism

$$
c_{A_{n-1}, \Delta}^{i_{1}, i_{2}, i_{3}, i_{4}}=\left(\left(t_{i_{1}}-t_{i_{4}}\right)\left(t_{i_{2}}-t_{i_{3}}\right):\left(t_{i_{1}}-t_{i_{3}}\right)\left(t_{i_{2}}-t_{i_{4}}\right)\right): U\left(A_{n-1}\right) \rightarrow \mathbb{P}^{1}
$$

depending on the choice of an ordering $I=\left\{i_{1}, i_{2}, i_{3}, i_{4}\right\}$ or equivalently a simple system $\alpha_{i_{1}, i_{2}}, \alpha_{i_{2}, i_{3}}, \alpha_{i_{3}, i_{4}}$ in $\Delta \cong A_{3}$. The morphisms $c_{A_{n-1}, \Delta}^{i_{1}, i_{2}, i_{3}, i_{4}}$ for different orderings $I=\left\{i_{1}, \ldots, i_{4}\right\}$ are related by isomorphisms of $\mathbb{P}^{1}$ : for a permutation $\sigma$ there is an isomorphism $\varphi_{\sigma}$ of $\mathbb{P}^{1}$ such that $c_{A_{n-1}, \Delta}^{\sigma\left(i_{1}\right), \sigma\left(i_{2}\right), \sigma\left(i_{3}\right), \sigma\left(i_{4}\right)}=\varphi_{\sigma} \circ c_{A_{n-1}, \Delta}^{i_{1}, i_{2}, i_{3}, i_{4}}$, where $\varphi_{\sigma}$ for generators of the permutation group is given by the following matrices $M_{\sigma}$ :

$$
M_{\left(i_{1} i_{2}\right)}=\left(\begin{array}{ll}
0 & 1 \\
1 & 0
\end{array}\right), \quad M_{\left(i_{1} i_{3}\right)}=\left(\begin{array}{cc}
-1 & 1 \\
0 & 1
\end{array}\right), \quad M_{\left(i_{3} i_{4}\right)}=\left(\begin{array}{ll}
0 & 1 \\
1 & 0
\end{array}\right)
$$

EXAMPLE $3.7 . \bar{X}_{A_{3}, A_{3}} \cong \mathbb{P}^{1}$.

For each ordering $\{1, \ldots, 4\}=\left\{i_{1}, i_{2}, i_{3}, i_{4}\right\}$ we have a copy of $\mathbb{P}^{1}$ with homogeneous coordinates $x_{i_{4}, 0}^{i_{1}, i_{2}, i_{3}}, x_{i_{4}, 1}^{i_{1}, i_{2}, i_{3}}$ and the morphism $c_{A_{3}, A_{3}}^{i_{1}, i_{2}, i_{3}, i_{4}}: U\left(A_{3}\right) \rightarrow \mathbb{P}^{1}$ defined in (11). Let $X_{A_{3}, A_{3}} \subset \prod_{i \in S_{4}} \mathbb{P}^{1}$ the image of $c_{A_{3}, A_{3}}=\prod_{i \in S_{4}} c_{A_{3}, A_{3}}^{i_{1}, i_{2}, i_{3}, i_{4}}$, then $X_{A_{3}, A_{3}} \cong \mathbb{P}^{1} \backslash$ $\{(0: 1),(1: 0)\}$ and its closure $\bar{X}_{A_{3}, A_{3}} \cong \mathbb{P}^{1}$ via projections to the factors. Using (12), $\bar{X}_{A_{3}, A_{3}}$ can be described as the subscheme of $\prod_{i \in S_{4}} \mathbb{P}^{1}$ defined by equations of the form

$$
\begin{aligned}
& x_{i_{4}, 0}^{i_{2}, i_{1}, i_{3}} x_{i_{4}, 0}^{i_{1}, i_{2}, i_{3}}=x_{i_{4}, 1}^{i_{2}, i_{1}, i_{3}} x_{i_{4}, 1}^{i_{1}, i_{2}, i_{3}} \\
& x_{i_{4}, 0}^{i_{3}, i_{2}, i_{1}} x_{i_{4}, 1}^{i_{1}, i_{2}, i_{3}}=x_{i_{4}, 1}^{i_{3}, i_{2}, i_{1}}\left(x_{i_{4}, 1}^{i_{1}, i_{2}, i_{3}}-x_{i_{4}, 0}^{i_{1}, i_{2}, i_{3}}\right) \\
& x_{i_{3}, 0}^{i_{1}, i_{2}, i_{4}} x_{i_{4}, 0}^{i_{1}, i_{2}, i_{3}}=x_{i_{3}, 1}^{i_{1}, i_{2}, i_{4}} x_{i_{4}, 1}^{i_{1}, i_{2}, i_{3}}
\end{aligned}
$$

There are the factorisations $c_{A_{3}, A_{3}}^{i_{1}, i_{2}, i_{3}, i_{4}}=\varphi_{A_{3}}^{i_{1}, i_{2}, i_{3}, i_{4}} \circ c_{A_{3}, A_{3}}: U\left(A_{3}\right) \rightarrow \bar{X}_{A_{3}, A_{3}} \stackrel{\sim}{\rightarrow} \mathbb{P}^{1}$ where $\varphi_{A_{3}}^{i_{1}, i_{2}, i_{3}, i_{4}}: \bar{X}_{A_{3}, A_{3}} \stackrel{\sim}{\rightarrow} \mathbb{P}^{1}$ is induced by the projection onto the corresponding factor.

In general for root systems $\Delta \cong A_{3}$ in $A_{n-1}$ we have a morphism $c_{A_{n-1}, \Delta}$ : $U\left(A_{n-1}\right) \rightarrow U(\Delta) \stackrel{c_{\Delta, \Delta}}{\longrightarrow} \bar{X}_{\Delta, \Delta}$ such that

$$
c_{A_{n-1}, \Delta}^{i_{1}, i_{2}, i_{3}, i_{4}}=\varphi_{\Delta}^{i_{1}, i_{2}, i_{3}, i_{4}} \circ c_{A_{n-1}, \Delta}: U\left(A_{n-1}\right) \rightarrow U(\Delta) \rightarrow \bar{X}_{\Delta, \Delta} \stackrel{\sim}{\rightarrow} \mathbb{P}^{1} .
$$

DEFinition 3.8. We denote the image $X_{A_{n-1}, A_{3}}=\left(\prod_{A_{3} \cong \Delta \subseteq A_{n-1}} c_{A_{n-1}, \Delta}\right)\left(U\left(A_{n-1}\right)\right)$ and define the cross-ratio variety $\bar{X}_{A_{n-1}, A_{3}}$ for root systems of type $A_{3}$ in $A_{n-1}$ as the closure

$$
\bar{X}_{A_{n-1}, A_{3}}=\bar{X}_{A_{n-1}, A_{3}} \subseteq \prod_{A_{3} \cong \Delta \subseteq A_{n-1}} \bar{X}_{\Delta, \Delta} \cong \prod_{A_{3} \cong \Delta \subseteq A_{n-1}} \mathrm{P}^{1} .
$$

Let $\bar{c}_{A_{n-1}, \Delta}: \bar{X}_{A_{n-1}, A_{3}} \rightarrow \bar{X}_{\Delta, \Delta}$ be the morphisms induced by the projection on the factors of the product.

In a similar way we can construct a scheme from the set of root subsystems of type $A_{1}$ in $A_{n-1}$. We have the $n$-dimensional projective space $\mathbb{P}\left(L\left(A_{n-1}\right)\right)$ with homogeneous coordinates $t_{1}, \ldots, t_{n}$ corresponding to the basis $e_{1}, \ldots, e_{n}$. The open subscheme $T\left(A_{n-1}\right)=\mathbb{P}\left(L\left(A_{n-1}\right)\right) \backslash \bigcup_{i}\left\{t_{i}=0\right\} \subset \mathbb{P}\left(L\left(A_{n-1}\right)\right)$ is the algebraic torus with character lattice $M\left(A_{n-1}\right)$. For root subsystems $A_{1} \cong\left\{ \pm \alpha_{i, j}\right\} \subseteq A_{n-1}$ we have morphisms

$$
c_{A_{n-1},\left\{ \pm \alpha_{i, j}\right\}}^{i, j}=\left(t_{i}: t_{j}\right): T\left(A_{n-1}\right) \rightarrow \mathbb{P}^{1}
$$

depending on the choice of an ordering of $\{i, j\}$ or equivalently on the choice of a simple system $\alpha_{i, j}$ in $\left\{ \pm \alpha_{i, j}\right\}$. The morphisms $c_{A_{n-1},\left\{ \pm \alpha_{i, j}\right\}}^{i, j}$ and $c_{A_{n-1},\left\{ \pm \alpha_{i, j}\right\}}^{j, i}$ for the two different orderings of $\{i, j\}$ are related by the isomorphism of $\mathbb{P}^{1}$ given by the matrix $\left(\begin{array}{ll}0 & 1 \\ 1 & 0\end{array}\right)$. 
EXAMPLE 3.9. $\bar{X}_{A_{1}, A_{1}} \cong \mathbb{P}^{1}$.

In the case of $A_{1}=\left\{ \pm \alpha_{i, j}\right\}$ for each ordering of $\{i, j\}$ we have a copy of $\mathbb{P}^{1}$ with homogeneous coordinates $x_{i, 0}^{j}, x_{i, 1}^{j}$ and a morphism $c_{A_{1}, A_{1}}^{i, j}: U\left(A_{3}\right) \rightarrow \mathbb{P}^{1}$. Let $X_{A_{1}, A_{1}} \subset$ $\mathbb{P}^{1} \times \mathbb{P}^{1}$ be the image of $c_{A_{1}, A_{1}}=c_{A_{1}, A_{1}}^{i, j} \times c_{A_{1}, A_{1}}^{j, i}$, then $X_{A_{1}, A_{1}} \cong \mathbb{P}^{1} \backslash\{(0: 1),(1: 0)\}$ and its closure $\bar{X}_{A_{1}, A_{1}} \cong \mathbb{P}^{1}$ via projections to the factors. $\bar{X}_{A_{1}, A_{1}}$ can be described as the subscheme of $\mathbb{P}^{1} \times \mathbb{P}^{1}$ defined by the equation $x_{j, 0}^{i}, x_{i, 0}^{j}=x_{j, 1}^{i} x_{i, 1}^{j}$. The morphism $c_{A_{1}, A_{1}}^{i, j}$ factors as $T\left(A_{1}\right) \stackrel{c_{A_{1}, A_{1}}}{\longrightarrow} \bar{X}_{A_{1}, A_{1}} \stackrel{\sim}{\rightarrow} \mathbb{P}^{1}$, where the last morphism is induced by the projection onto the corresponding factor.

For root subsystems $A_{1} \cong\left\{ \pm \alpha_{i, j}\right\} \subseteq A_{n-1}$ the projection $T\left(A_{n-1}\right) \rightarrow T\left(\left\{ \pm \alpha_{i, j}\right\}\right)$ composed with $c_{\left\{ \pm \alpha_{i, j}\right\},\left\{ \pm \alpha_{i, j}\right\}}$ gives the morphism

$$
c_{A_{n-1},\left\{ \pm \alpha_{i, j}\right\}}: T\left(A_{n-1}\right) \rightarrow T\left(\left\{ \pm \alpha_{i, j}\right\}\right) \rightarrow \bar{X}_{\left\{ \pm \alpha_{i, j}\right\},\left\{ \pm \alpha_{i, j}\right\}}
$$

that satisfies $c_{\left\{ \pm \alpha_{i, j}\right\},\left\{ \pm \alpha_{i, j}\right\}}^{i, j} \circ c_{A_{n-1},\left\{ \pm \alpha_{i, j}\right\}}=c_{A_{n-1},\left\{ \pm \alpha_{i, j}\right\}}^{i, j}: T\left(A_{n-1}\right) \rightarrow \mathbb{P}^{1}$.

The morphisms $c_{A_{n-1},\left\{ \pm \alpha_{i, j}\right\}}: T\left(A_{n-1}\right) \stackrel{\rightarrow}{\rightarrow} X_{\left\{ \pm \alpha_{i, j}\right\},\left\{ \pm \alpha_{i, j}\right\}} \cong \mathbb{G}_{m}$ are homomorphisms of algebraic tori. Their product, the homomorphism of tori $\prod_{A_{1} \cong\left\{ \pm \alpha_{i, j}\right\} \subseteq A_{n-1}} c_{A_{n-1},\left\{ \pm \alpha_{i, j}\right\}}$, embeds $T\left(A_{n-1}\right)$ into the open dense torus of $\prod_{A_{1} \cong\left\{ \pm \alpha_{i, j}\right\} \subseteq A_{n-1}} \bar{X}_{\left\{ \pm \alpha_{i, j}\right\},\left\{ \pm \alpha_{i, j}\right\}}$, we denote its image $X_{A_{n-1}, A_{1}}$. The closure

$$
\bar{X}_{A_{n-1}, A_{1}}=\overline{X_{A_{n-1}, A_{1}}} \subseteq \prod_{A_{3} \cong\left\{ \pm \alpha_{i, j}\right\} \subseteq A_{n-1}} \bar{X}_{\left\{ \pm \alpha_{i, j}\right\},\left\{ \pm \alpha_{i, j}\right\}} \cong \prod_{A_{1} \cong\left\{ \pm \alpha_{i, j}\right\} \subseteq A_{n-1}} \mathbb{P}^{1}
$$

coincides with the toric variety $X\left(A_{n-1}\right)$ associated with the root system $A_{n-1}$, i.e. its fan is the fan of Weyl chambers of the root system $A_{n-1}$ in the lattice $N\left(A_{n-1}\right)$, because on the open dense torus $T\left(A_{n-1}\right) \subset X\left(A_{n-1}\right)$ the morphisms $c_{A_{n-1},\left\{ \pm \alpha_{i, j}\right\}}$ coincide with the morphisms

$$
\gamma_{\left\{ \pm \alpha_{i j}\right\}}: X\left(A_{n-1}\right) \rightarrow X\left(A_{1}\right) \cong \mathbb{P}^{1}
$$

for inclusions of root subsystems $\left\{ \pm \alpha_{i j}\right\} \subseteq A_{n-1}$ of type $A_{1}$ and the morphism $\prod_{\left\{ \pm \alpha_{i j}\right\}} \gamma_{\left\{ \pm \alpha_{i j}\right\}}$ is a closed embedding (see [3] or theorem 3.11 below).

Already in $[23,(2.6 .3)]$ the Losev-Manin moduli space $\bar{L}_{n}$ has been identified as the smooth projective toric variety associated with the $(n-1)$-dimensional permutohedron, which coincides with $X\left(A_{n-1}\right)$. In [3] an alternative proof that the functor $\bar{L}_{n}$ is representable and the fine moduli space $\bar{L}_{n}$ is isomorphic to the smooth projective toric variety $X\left(A_{n-1}\right)$ is given after a systematic study of toric varieties $X(R)$ associated with root systems $R$, their functorial properties with respect to maps of root systems and their functor of points, and it is shown that the morphisms $\gamma_{R}: X\left(A_{n-1}\right) \rightarrow$ $X(R) \cong X\left(A_{k-1}\right)$ corresponding to inclusions of root systems $A_{k-1} \cong R \subset A_{n-1}$ can be identified with the contraction morphisms $\gamma_{I}: \bar{L}_{n} \rightarrow \bar{L}_{I} \cong \bar{L}_{k}$ determined by subsets $I \subset\{1, \ldots, n\},|I|=k$.

EXAMPLE 3.10. $\bar{L}_{2} \cong \mathbb{P}^{1}$.

Each $i \in\{1,2\}$ defines via the line bundle $\mathcal{O}_{C}\left(s_{i}\right)$ a contraction of any stable 2-pointed chain $\left(C \rightarrow Y, s_{0}, s_{\infty}, s_{1}, s_{2}\right)$ over a scheme $Y$ to a trivial $\mathbb{P}^{1}$-bundle over $Y$ with three pairwise disjoint sections $s_{0}^{i}, s_{\infty}^{i}, s_{i}^{i}$ and a fourth section $s_{j}^{i}$. We can choose homogeneous coordinates $x_{j, 0}^{i}, x_{j, 1}^{i}$ such that $s_{0}^{i}=(0: 1), s_{\infty}^{i}=(1: 0), s_{i}^{i}=(1: 1)$. The remaining section $s_{j}^{i}=\left(s_{j, 0}^{i}: s_{j, 1}^{i}\right)$ of $\mathbb{P}_{Y}^{1}$ determines an isomorphism $\bar{L}_{2} \cong \mathbb{P}^{1}$. Since $\left(s_{j, 0}^{i}: s_{j, 1}^{i}\right)=\left(s_{i, 1}^{j}: s_{i, 0}^{j}\right), \bar{M}_{0,4}$ can be embedded in $\mathbb{P}^{1} \times \mathbb{P}^{1}$ as the subscheme defined by the same equation $x_{j, 0}^{i}, x_{i, 0}^{j}=x_{j, 1}^{i} x_{i, 1}^{j}$ as in example 3.9 , and the two projections induce the above isomorphisms $\bar{M}_{0,4} \cong \mathbb{P}^{1}$ corresponding to the two choices of an ordering of $\{1,2\}$. 
THEOREM 3.11 ([3]). The morphism

$$
\prod_{\{i, j\}} \gamma_{\{i, j\}}: \bar{L}_{n} \rightarrow \prod_{\{i, j\}} \bar{L}_{\{i, j\}} \text { or equivalently } \prod_{\left\{ \pm \alpha_{i, j}\right\}} \gamma_{\left\{ \pm \alpha_{i, j}\right\}}: X\left(A_{n-1}\right) \rightarrow \prod_{\left\{ \pm \alpha_{i, j}\right\}} X\left(\left\{ \pm \alpha_{i, j}\right\}\right),
$$

where the product is over all subsets $I=\{i, j\} \subset\{1, \ldots, n\},|I|=2$ or equivalently over all root subsystems $A_{1} \cong\left\{ \pm \alpha_{i, j}\right\} \subseteq A_{n-1}$, is a closed embedding. Its image in $\prod_{\{i, j\}} \bar{L}_{\{i, j\}}=\prod_{\left\{ \pm \alpha_{i, j}\right\}} X\left(\left\{ \pm \alpha_{i, j}\right\}\right)$ is given by the equations of the form

$$
x_{i, 0}^{j} x_{k, 0}^{i} x_{k, 1}^{j}=x_{i, 1}^{j} x_{k, 1}^{i} x_{k, 0}^{j}
$$

for all subsets $J=\{i, j, k\} \subseteq\{1, \ldots, n\},|J|=3$ or equivalently all root subsystems $A_{2} \cong\left\{ \pm \alpha_{i, j}, \pm \alpha_{j, k}, \pm \alpha_{i, k}\right\} \subseteq A_{n-1}$, where $x_{j, 0}^{i}, x_{j, 1}^{i}$ are the homogeneous coordinates of $\bar{L}_{\{i, j\}}=X\left(\left\{ \pm \alpha_{i, j}\right\}\right)$ as defined in examples 3.9 and 3.10. The functor of points of $\bar{L}_{n}=X\left(A_{n-1}\right)$ is isomorphic to the contravariant functor

$$
Y \mapsto\left\{\begin{array}{l|l}
\text { families of sections } & \forall i: s_{i, 0}^{i}=s_{i, 1}^{i} \\
\left(s_{j}^{i}: Y \rightarrow \mathbb{P}_{Y}^{1}\right)_{i, j \in\{1, \ldots, n\}} & \forall i, j, k: s_{i, 0}^{j} s_{k, 0}^{i} s_{k, 1}^{j}=s_{i, 1}^{j} s_{k, 1}^{i} s_{k, 0}^{j}
\end{array}\right\}
$$

Similarly, we can describe $\bar{M}_{0, n}$ and its embedding into a product of $\mathbb{P}^{1}$ 's in terms of the root system $A_{n-1}$. First we consider the case of $\bar{M}_{0,4}$.

EXAMPLE $3.12 \cdot \bar{M}_{0,4} \cong \mathbb{P}^{1}$.

We choose a permutation $I=\{1, \ldots, 4\}=\left\{i_{1}, \ldots, i_{4}\right\}$. Three sections $s_{i_{1}}, s_{i_{2}}, s_{i_{3}}$ define a contraction of any stable 4 -pointed curve $\left(C \rightarrow Y, s_{i_{1}}, s_{i_{2}}, s_{i_{3}}, s_{i_{4}}\right)$ to a trivial $\mathbb{P}^{1}$-bundle over $Y$ via the line bundle $\omega_{C / Y}\left(s_{i_{1}}+s_{i_{2}}+s_{i_{3}}\right)$ (cf. [21]). Choosing homogeneous coordinates $x_{i_{4}, i_{1}, i_{3}}^{i_{1}, i_{3}}, x_{i_{4}, 1}^{i_{1}, i_{2}, i_{3}}$ of $\mathbb{P}_{Y}^{1}$ such that $s_{i_{1}}=(0: 1), s_{i_{2}}=(1: 0)$, $s_{i_{3}}=(1: 1)$, the remaining section $s_{i_{4}}=\left(s_{i_{4}, 0}^{i_{1}, i_{2}, i_{3}}: s_{i_{4}, 1}^{i_{1}, i_{2}, i_{3}}\right)$ of $\mathbb{P}_{Y}^{1}$ yields an isomorphism $\bar{M}_{0,4} \cong \mathbb{P}^{1}$. Under permutations of $I$ this fourth section is transformed by isomorphisms of $\mathbb{P}^{1}$ as in (12), so the sections in these coordinates are related by

$$
\begin{aligned}
& s_{i_{4}, 0}^{i_{2}, i_{1}, i_{3}} s_{i_{4}, 0}^{i_{1}, i_{2}, i_{3}}=s_{i_{i}, 1}^{i_{2}, i_{1}, i_{3}} s_{i_{4}, 1}^{i_{1}, i_{2}, i_{3}} \\
& s_{i_{4}, 0}^{i_{3}, i_{2}, i_{1}} s_{i_{4}, 1}^{i_{1}, i_{2}, i_{3}}=s_{i_{4}, 1}^{i_{3}, i_{2}, i_{1}}\left(s_{i_{4}, 1}^{i_{1}, i_{2}, i_{3}}-s_{i_{4}, 0}^{i_{1}, i_{2}, i_{3}}\right)
\end{aligned}
$$

for permutations of the upper indices and by

$$
s_{i_{4}, 0}^{i_{1}, i_{2}, i_{3}} s_{i_{3}, 0}^{i_{1}, i_{2}, i_{4}}=s_{i_{4}, 1}^{i_{1}, i_{2}, i_{3}} s_{i_{3}, 1}^{i_{1}, i_{2}, i_{4}} .
$$

Therefore the moduli space $\bar{M}_{0,4}$ can be described as the subscheme in $\prod_{i \in S_{4}} \mathbb{P}^{1}$ by equations of the form (13), where each projection induces the above isomorphism $\bar{M}_{0,4} \cong \mathbb{P}^{1}$ corresponding to a choice of an ordering of $I$.

The space $U\left(A_{n-1}\right)=\mathbb{A}\left(L\left(A_{n-1}\right)\right) \backslash \bigcup_{i \neq j}\left\{t_{i}=t_{j}\right\}$ parametrises $n$ distinct points in $\mathbb{A}^{1}=\mathbb{P}^{1} \backslash\{(1: 0)\}$, so its tautological family determines a morphism $\psi: U\left(A_{n-1}\right) \rightarrow M_{0, n}$. A point $\left(a_{1}, a_{2}, a_{3}, a_{4}\right) \in U\left(A_{3}\right)$, considered as tuple $\left.\left(\left(a_{1}: 1\right),\left(a_{2}: 1\right),\left(a_{3}: 1\right),\left(a_{4}: 1\right)\right)\right)$ of points in $\mathbb{P}^{1}$, can be transformed by the matrix $\left(\begin{array}{ll}-\left(a_{2}-a_{3}\right) & a_{1}\left(a_{2}-a_{3}\right) \\ -\left(a_{1}-a_{3}\right) & a_{2}\left(a_{1}-a_{3}\right)\end{array}\right)$ into the tuple $\left((0: 1),(1: 0),(1: 1),\left(\left(a_{1}-a_{4}\right)\left(a_{2}-a_{3}\right)\right.\right.$ : $\left.\left.\left(a_{2}-a_{4}\right)\left(a_{1}-a_{3}\right)\right)\right)$ ) (compare to $\left.(11)\right)$. We obtain the following result:

Lemma 3.13. Let $I=\left\{i_{1}, i_{2}, i_{3}, i_{4}\right\} \subseteq\{1, \ldots, n\},|I|=4$ and $A_{3} \cong \Delta=\left\{\alpha_{i, j} \mid i, j \in\right.$ $I\} \subseteq A_{n-1}$ the corresponding root subsystem. We identify $\bar{M}_{0, I} \cong \mathbb{P}^{1}$ as in example 3.12 by $\left(C \rightarrow Y,\left(s_{i}\right)_{i}\right) \mapsto\left(s_{i_{4}}: Y \rightarrow \mathbb{P}_{Y}^{1}\right)$ after contracting a curve $C \rightarrow Y$ over $Y$ to $\mathbb{P}_{Y}^{1}$ and choosing a basis such that the sections $s_{i_{1}}, s_{i_{2}}, s_{i_{3}}$ become $(0: 1),(1: 0)$, (1: 1$)$, and denote the composition $\gamma_{I}^{i_{1}, i_{2}, i_{3}, i_{4}}: \bar{M}_{0, n} \stackrel{\gamma_{I} \rightarrow \bar{M}_{0, I}}{\rightarrow} \stackrel{\sim}{\rightarrow} \mathbb{P}^{1}$. This relates to the morphism $c_{A_{n-1}, \Delta}^{i_{1}, i_{2}, i_{3}, i_{4}}: U\left(A_{n-1}\right) \rightarrow U(\Delta) \rightarrow \bar{X}_{\Delta, \Delta} \stackrel{\sim}{\rightarrow} \mathbb{P}^{1}$ of (11) as follows:

$$
c_{A_{n-1}, \Delta}^{i_{1}, i_{2}, i_{3}, i_{4}}=\gamma_{I}^{i_{1}, i_{2}, i_{3}, i_{4}} \circ \psi .
$$


The following theorem is based on the closed embeddings of $\bar{M}_{0, n}$ and its universal curve in products of $\mathbb{P}^{1}$ 's described in $[10$, Prop. 4]. We use this to deduce a characterisation of the functor of points of $\bar{M}_{0, n}$, add the interpretation in terms of root systems and the link to the cross-ratio varieties using lemma 3.13.

THEOREM 3.14. The morphism

$$
\prod_{|I|=4} \gamma_{I}: \bar{M}_{0, n} \rightarrow \prod_{|I|=4} \bar{M}_{0, I}
$$

where the product is over all subsets $I \subseteq\{1, \ldots, n\},|I|=4$, is a closed embedding. This embedding induces an isomorphism $\bar{M}_{0, n} \rightarrow \bar{X}_{A_{n-1}, A_{3}}$ and can be identified with the closed embedding

$$
\prod_{A_{3} \cong \Delta \subseteq A_{n-1}} \bar{c}_{A_{n-1}, \Delta}: \bar{X}_{A_{n-1}, A_{3}} \rightarrow \prod_{A_{3} \cong \Delta \subseteq A_{n-1}} \bar{X}_{\Delta, \Delta},
$$

where the product is over all root subsystems $\Delta$ isomorphic to $A_{3}$. The image of $\bar{M}_{0, n} \cong \bar{X}_{A_{n-1}, A_{3}}$ in $\prod_{I} \bar{M}_{0, I} \cong \prod_{\Delta} \bar{X}_{\Delta, \Delta}$ is given by the equations of the form

$$
x_{i_{4}, 0}^{i_{1}, i_{2}, i_{3}} x_{i_{5}, 1}^{i_{1}, i_{2}, i_{3}} x_{i_{5}, 0}^{i_{1}, i_{2}, i_{4}}=x_{i_{4}, 1}^{i_{1}, i_{2}, i_{3}} x_{i_{5}, 0}^{i_{1}, i_{2}, i_{3}} x_{i_{5}, 1}^{i_{1}, i_{2}, i_{4}}
$$

for all subsets $J \subseteq\{1, \ldots, n\},|J|=5$ or equivalently all root subsystems $A_{4} \cong$ $\Gamma \subseteq A_{n-1}$, where the homogeneous coordinates $x_{i_{4}, 0}^{i_{1}, i_{2}, i_{3}}, x_{i_{4}, 1}^{i_{1}, i_{2}, i_{3}}$ are defined as in examples 3.7 and 3.12, and homogeneous coordinates corresponding to different permutations of a set $I \subset\{1, \ldots, n\},|I|=4$ for the same $\bar{M}_{0, I}=\bar{X}_{\Delta, \Delta}$ are related by equations (13). The functor of points of $\bar{M}_{0, n}$ is isomorphic to the contravariant functor

$$
\begin{aligned}
& Y \mapsto \\
& \left\{\begin{array}{l|l}
\text { families of sections } & \forall T=\left\{i_{1}, i_{2}, i_{3}\right\},|T|=3: \\
s_{i_{4}, i_{2}, i_{3}}^{i_{1}} Y \rightarrow \mathbb{P}_{Y}^{1} & s_{i_{1}, i_{2}, i_{3}}^{i_{1}}=(0: 1), s_{i_{2}}^{i_{1}, i_{2}, i_{3}}=(1: 0), s_{i_{3}}^{i_{1}, i_{2}, i_{3}}=(1: 1) \\
\text { for } i_{1}, i_{2}, i_{3}, i_{4} \in\{1, \ldots, n\} & \forall I=\left\{i_{1}, i_{2}, i_{3}, i_{4}\right\},|I|=4: \\
\text { such that }\left|\left\{i_{1}, i_{2}, i_{3}\right\}\right|=3 & \text { equations }(15) \text { and }(16) \text { hold } \\
& \forall J=\left\{i_{1}, i_{2}, i_{3}, i_{4}, i_{5}\right\},|J|=5: \\
& s_{i_{4}, 0}^{i_{1}, i_{2}, i_{3}} s_{i_{5}, 1}^{i_{1}, i_{2}, i_{3}} s_{i_{5}, 0}^{i_{1}, i_{2}, i_{4}}=s_{i_{4}, 1}^{i_{1}, i_{2}, i_{3}} s_{i_{5}, 0}^{i_{1}, i_{2}, i_{3}} s_{i_{5}, 1}^{i_{1}, i_{2}, i_{4}}
\end{array}\right\}
\end{aligned}
$$

REMARK 3.15. The stable $n$-pointed tree can be reconstructed from data as in (17) over an $S$-scheme $Y$ as the curve $C \subseteq\left(\prod_{T} \mathbb{P}^{1}\right)_{Y}$, where the product is over subsets $T \subseteq\{1, \ldots, n\}$ such that $|T|=3$, defined by the equations

$$
s_{i_{4}, 0}^{i_{1}, i_{2}, i_{3}} x_{1}^{i_{1}, i_{2}, i_{3}} x_{0}^{i_{1}, i_{2}, i_{4}}=s_{i_{4}, 1}^{i_{1}, i_{2}, i_{3}} x_{0}^{i_{1}, i_{2}, i_{3}} x_{1}^{i_{1}, i_{2}, i_{4}},
$$

where $x_{0}^{i_{1}, i_{2}, i_{3}}, x_{1}^{i_{1}, i_{2}, i_{3}}$ are the homogeneous coordinates of the factor corresponding to $T=\left\{i_{1}, i_{2}, i_{3}\right\}$ and related under permutations of elements of $T$ by equations as the first two in (13) leaving out the lower index, and the sections are given as $s_{k}=\left(s_{k, 0}^{T}: s_{k, 1}^{T}\right)_{T}: Y \rightarrow C \subseteq\left(\prod_{T} \mathbb{P}^{1}\right)_{Y}$, where $\left(s_{k, 0}^{T}: s_{k, 1}^{T}\right)=\left(s_{k, 0}^{i_{1}, i_{2}, i_{3}}: s_{k, 1}^{i_{1}, i_{2}, i_{3}}\right)$ for $T=\left\{i_{1}, i_{2}, i_{3}\right\}$ with respect to the coordinates $x_{0}^{i_{1}, i_{2}, i_{3}}, x_{1}^{i_{1}, i_{2}, i_{3}}$. This follows easily from theorem 3.14 and the fact that $\bar{M}_{0, n+1} \rightarrow \bar{M}_{0, n}$ forms the universal $n$-pointed tree over $\bar{M}_{0, n}$. One may also compare this to proposition 2.16.

3.3. Losev-Manin And GrothendiecK-KNUdSEn MOdUli SPACES AS Limits of MODULI SPACES OF QUIVER REPRESENTATIONS. We relate moduli spaces of representations of $P_{n}$ to the Losev-Manin moduli spaces $\bar{L}_{n}$.

For $i \in\{1, \ldots, n\}$ we define the weight $\theta^{i} \in \Delta^{1} \times \Delta^{n-1} \subset H\left(P_{n}\right)$ by $\eta_{1}^{i}, \eta_{2}^{i}=-\frac{1}{2}$, $\theta_{i}^{i}=1-\varepsilon$ and $\theta_{j}^{i}=\frac{\varepsilon}{n-1}$ for $j \neq i$, where $1 \gg \varepsilon>0$. The weights $\theta^{i}$ are generic 
and any free representation $V^{\theta^{i}}$ that is $\theta^{i}$-stable has the property that, considered as tuple $\left(s_{j}^{\theta^{i}}\right)_{j}$ of sections of $\mathbb{P}^{1}, s_{i}^{\theta^{i}}$ does not meet $(0: 1)$ and $(1: 0)$. The moduli spaces $\mathcal{M}^{\theta^{i}}\left(P_{n}\right)$ are isomorphic to $\left(\mathbb{P}^{1}\right)^{n-1}$.

THEOREM 3.16. There is an isomorphism

$$
\bar{L}_{n} \cong \lim _{\theta} \mathcal{M}^{\theta}\left(P_{n}\right) \text {. }
$$

Proof. By proposition $2.15 \lim _{\theta} \mathcal{M}^{\theta}\left(P_{n}\right) \cong \operatorname{Lim}\left(P_{n}\right)$. Consider the functor $\operatorname{Lim}^{\prime}\left(P_{n}\right)$ defined by

$$
Y \mapsto\left\{\left(\varphi_{V^{\theta^{i}}}\right)_{i} \in \prod_{i} \mathcal{M}^{\theta^{i}}\left(P_{n}\right)(Y) \mid \forall i, j, k, l: s_{k, 0}^{\theta^{i}} s_{l, 1}^{\theta^{i}} s_{k, 1}^{\theta^{j}} s_{l, 0}^{\theta^{j}}=s_{k, 1}^{\theta^{i}} s_{l, 0}^{\theta^{i}} s_{k, 0}^{\theta^{j}} s_{l, 1}^{\theta^{j}}\right\}
$$

where $\varphi_{V^{\theta^{i}}}: Y \rightarrow \mathcal{M}^{\theta}(Q)$ is the morphism determined by a $\theta^{i}$-stable representation $V^{\theta^{i}}$ over $Y$, and the representations $V^{\theta^{i}}$ are considered as tuples of sections $\left(s_{k}^{\theta^{i}}\right)_{k}$ of $\mathrm{P}_{Y}^{1} \rightarrow Y$.

We claim that the natural morphism of functors $\operatorname{Lim}\left(P_{n}\right) \rightarrow \operatorname{Lim}^{\prime}\left(P_{n}\right)$ that arises by restricting elements and relations is an isomorphism. The property

$$
\operatorname{int}\left(\Theta\left(V^{\theta}(y)\right) \cap \Theta\left(V^{\theta^{\prime}}(y)\right)\right) \neq \varnothing \Longleftrightarrow V^{\theta} \cong V^{\theta^{\prime}} \text { in a neighbourhood of } y
$$

of $\left(V^{\theta}\right)_{\theta} \in \operatorname{Lim}\left(P_{n}\right)(Y)$ follows from the equations relating $V^{\theta}, V^{\theta^{\prime}}$ and thus also holds for elements of $\operatorname{Lim}^{\prime}\left(P_{n}\right)(Y)$ : by proposition 2.16.(c) we have an isomorphism $V^{\theta} \cong$ $V^{\theta^{\prime}}$ on an open set, and for $y$ in its complement there are $J, J^{\prime}$ such that $\left(s_{j}^{\theta}(y)\right)_{j \in J}$ coincide, $\left(s_{j}^{\theta^{\prime}}(y)\right)_{j \in J^{\prime}}$ coincide and $\left(J \cup J^{\prime}\right)^{\complement}=\varnothing$, which implies that $\Theta\left(V^{\theta}(y)\right)$ and $\Theta\left(V^{\theta^{\prime}}(y)\right)$ are separated by a wall. By this property for elements of $\operatorname{Lim}\left(P_{n}\right)(Y)$ (resp. $\left.\operatorname{Lim}^{\prime}\left(P_{n}\right)(Y)\right)$ the polytopes $\Theta\left(V^{\theta}(y)\right)$ (resp. $\left.\Theta\left(V^{\theta^{i}}(y)\right)\right)$ have either disjoint interiors or coincide. We construct the inverse morphism by showing that there is a unique way to extend an element $\left(V^{\theta^{i}}\right)_{i} \in \operatorname{Lim}^{\prime}\left(P_{n}\right)(Y)$ to an element $\left(V^{\theta}\right)_{\theta} \in \operatorname{Lim}\left(P_{n}\right)(Y)$. Such a $\left(V^{\theta}\right)_{\theta} \in \operatorname{Lim}\left(P_{n}\right)(Y)$ is uniquely determined by its restriction to $\operatorname{Lim}^{\prime}\left(P_{n}\right)(Y)$ because for any generic $\theta$ we have $\bigcup_{i}\left\{y \mid V^{\theta}(y) \theta^{i}\right.$-stable $\}=Y$. On the other hand we can extend a given $\left(V^{\theta^{i}}\right)_{i} \in \operatorname{Lim}^{\prime}\left(P_{n}\right)(Y)$ to an element of $\operatorname{Lim}\left(P_{n}\right)(Y)$ if for any generic $\theta$ we have $\bigcup_{i}\left\{y \mid V^{\theta^{i}}(y) \theta\right.$-stable $\}=Y$ or equivalently for all $y \in Y$ the polytopes $\Theta\left(V^{\theta^{i}}(y)\right)$ cover $\Delta^{1} \times \Delta^{n-1}$. The fibres $\left(V^{\theta^{i}}(y)\right)_{i}$ over a geometric point $y \in Y$ define a decomposition of $\Delta^{1} \times \Delta^{n-1}$ into polytopes: the polytopes $\Theta\left(V^{\theta^{i}}(y)\right)$ and possibly remaining parts. By remark 2.4 and lemma 2.6 each of the remaining parts is of the form

$$
P\left(J, J^{\prime}\right)=\left\{\theta \in \Delta^{1} \times \Delta^{n-1} \mid \sum_{i \in J} \theta_{i} \leqslant-\eta_{1}\right\} \cap\left\{\theta \in \Delta^{1} \times \Delta^{n-1} \mid \sum_{i \in J^{\prime}} \theta_{i} \leqslant-\eta_{2}\right\},
$$

i.e. bounded by walls $W_{J}$ and $W_{\left(J^{\prime}\right)^{\mathrm{C}}}$, for some $J, J^{\prime}$ such that $J \subsetneq\left(J^{\prime}\right)^{\complement}$. But any such $P\left(J, J^{\prime}\right)$ contains $\theta^{i}$ for $i \in\{1, \ldots, n\} \backslash\left(J \cup J^{\prime}\right)$. So already the polytopes $\Theta\left(V^{\theta^{i}}(y)\right)$ cover $\Delta^{1} \times \Delta^{n-1}$. Thus we have shown $\operatorname{Lim}\left(P_{n}\right) \cong \operatorname{Lim}^{\prime}\left(P_{n}\right)$.

By remark 2.17 we can restrict the equations $s_{k, 0}^{\theta^{i}} s_{l, 1}^{\theta^{i}} s_{k, 1}^{\theta^{j}} s_{l, 0}^{\theta^{j}}=s_{k, 1}^{\theta^{i}} s_{l, 0}^{\theta^{i}} s_{k, 0}^{\theta^{j}} s_{l, 1}^{\theta^{j}}$ in the functor $\operatorname{Lim}^{\prime}\left(P_{n}\right)$ relating $V^{\theta^{i}}, V^{\theta^{j}}$ to equations with $l=i$. Thus $\operatorname{Lim}^{\prime}\left(P_{n}\right)$ is isomorphic to the functor $\operatorname{Lim}^{\prime \prime}\left(P_{n}\right)$ defined by

$$
Y \mapsto\left\{\left(\varphi_{V^{\theta^{i}}}\right)_{i} \in \prod_{i} \mathcal{M}^{\theta^{i}}\left(P_{n}\right)(Y) \mid \forall i, j, k: s_{k, 0}^{\theta^{i}} s_{i, 1}^{\theta^{i}} s_{k, 1}^{\theta^{j}} s_{i, 0}^{\theta^{j}}=s_{k, 1}^{\theta^{i}} s_{i, 0}^{\theta^{i}} s_{k, 0}^{\theta^{j}} s_{i, 1}^{\theta^{j}}\right\}
$$

Each $\varphi_{V^{\theta^{i}}}$ is given by a family $\left(s_{j}^{\theta^{i}}\right)_{j=1, \ldots, n}$ of sections $s_{j}^{\theta^{i}}: Y \rightarrow \mathbb{P}_{Y}^{1}$ up to operation of $\mathbb{G}_{m}$ on $\mathbb{P}_{Y}^{1}$ fixing $(0: 1),(1: 0)$. Within its isomorphism class we can choose this 
family such that $s_{i}^{\theta^{i}}=(1: 1)$, then we denote $s_{j}^{i}=s_{j}^{\theta^{i}}$. The functor $\operatorname{Lim}^{\prime \prime}\left(P_{n}\right)$ is isomorphic to

$$
Y \mapsto\left\{\begin{array}{l|l}
\text { families }\left(s_{j}^{i}\right)_{i, j \in\{1, \ldots, n\}} & \forall i: s_{i, 0}^{i}=s_{i, 1}^{i} \\
\text { of sections } s_{j}^{i}: Y \rightarrow \mathbb{P}_{Y}^{1} & \forall i, j, k: s_{k, 0}^{i} s_{k, 1}^{j} s_{i, 0}^{j}=s_{k, 1}^{i} s_{k, 0}^{j} s_{i, 1}^{j}
\end{array}\right\}
$$

This functor is the same as the functor (14) in theorem 3.11.

In the same way we study the relation of moduli spaces of representations of $Q_{n}$ to the Grothendieck-Knudsen moduli spaces $\bar{M}_{0, n}$.

For $T \subseteq\{1, \ldots, n\},|T|=3$ we define the weight $\theta^{T}$ for the quiver $Q_{n}$ as $\theta_{i}^{T}=$ $\frac{2}{3}(1-\varepsilon)$ for $i \in T$ and $\theta_{i}^{T}=\frac{2}{n-3} \varepsilon$ for $i \notin T$, where $1 \gg \varepsilon>0$. The weights $\theta^{T}$ are generic and for any $\theta^{T}$-stable free representation $V^{\theta^{T}}$, considered as a tuple $\left(s_{j}^{\theta^{T}}\right)_{j}$ of sections of $\mathbb{P}^{1}$, the sections $\left(s_{i}^{\theta^{T}}\right)_{i \in T}$ are disjoint. The moduli spaces $\mathcal{M}^{\theta^{T}}\left(Q_{n}\right)$ are isomorphic to $\left(\mathbb{P}^{1}\right)^{n-3}$.

THEOREM 3.17. There is an isomorphism

$$
\bar{M}_{0, n} \cong \lim _{\theta} \mathcal{M}^{\theta}\left(Q_{n}\right) \text {. }
$$

Proof. By proposition $2.15 \lim _{\theta} \mathcal{M}^{\theta}\left(Q_{n}\right) \cong \operatorname{Lim}\left(Q_{n}\right)$. Consider the functor $\operatorname{Lim}^{\prime}\left(Q_{n}\right)$ defined by

$$
Y \mapsto\left\{\left(\varphi_{V^{\theta^{T}}}\right)_{T} \in \prod_{T} \mathcal{M}^{\theta^{T}}\left(Q_{n}\right)(Y) \mid \text { equations (8) hold }\right\}
$$

where $\varphi_{V^{\theta^{T}}}: Y \rightarrow \mathcal{M}^{\theta}(Q)$ is the morphism determined by a $\theta^{T}$-stable representation $V^{\theta^{T}}$ over $Y$, and the representations $V^{\theta^{T}}$ are considered as tuples of sections $\left(s_{k}^{\theta^{T}}\right)_{k}$ of $\mathrm{P}_{Y}^{1} \rightarrow Y$.

In the same way as in the proof in the case of $P_{n}$ we show that the natural morphism $\operatorname{Lim}\left(Q_{n}\right) \rightarrow \operatorname{Lim}^{\prime}\left(Q_{n}\right)$ is an isomorphism by showing that $\bigcup_{T}\left\{y \mid V^{\theta^{T}}(y) \theta\right.$-stable $\}=Y$ for all $\left(V^{\theta^{T}}\right)_{T}$ defining an element of $\operatorname{Lim}^{\prime}\left(Q_{n}\right)(Y)$ and all generic $\theta$. For such $\left(V^{\theta^{T}}\right)_{T}$ and fixed $y \in Y$ again the polytopes $\Theta\left(V^{\theta^{T}}(y)\right)$ either coincide or their interiors are disjoint, and $\Delta(2, n)$ decomposes into the polytopes $\Theta\left(V^{\theta^{T}}(y)\right)$ and remaining parts. Using remark 2.2 and lemma 2.3 we see that the remaining parts consist of polytopes bounded by walls $W_{\left\{J_{l}, J_{l}^{\mathrm{C}}\right\}}$ which do not intersect in the interior of $\Delta(2, n)$ and thus are of the form $\bigcap_{l}\left\{\theta \in \Delta(2, n) \mid \sum_{j \in J_{l}} \theta_{j} \leqslant 1\right\}$ for disjoint subsets $J_{l} \subset\{1, \ldots, n\}$, $2 \leqslant\left|J_{l}\right| \leqslant n-2$. Any such subset which is full-dimensional contains a $\theta^{T}$, thus $\bigcup_{T} \Theta\left(V^{\theta^{T}}(y)\right)=\Delta(2, n)$ for fixed $y \in Y$, which is equivalent to the above statement.

In the functor $\operatorname{Lim}^{\prime}\left(Q_{n}\right)$ the statement that equations 8 hold means that for all $T, T^{\prime}$ and for all $i, j$ locally over $\left\{y \mid s_{i}^{\theta^{T}}(y) \neq s_{j}^{\theta^{T}}(y), s_{i}^{\theta^{T^{\prime}}}(y) \neq s_{j}^{\theta^{T^{\prime}}}(y)\right\} \subseteq Y$, after a choice of coordinates such that $s_{i}^{\theta^{T}}, s_{i}^{\theta^{T^{\prime}}}=(0: 1)$ and $s_{j}^{\theta^{T}}, s_{j}^{\theta^{T^{\prime}}}=(1: 0)$, for all $k, l$ the equations

$$
s_{k, 0}^{\theta^{T}} s_{l, 1}^{\theta^{T}} s_{k, 1}^{\theta^{T^{\prime}}} s_{l, 0}^{\theta^{T^{\prime}}}=s_{k, 1}^{\theta^{T}} s_{l, 0}^{\theta^{T}} s_{k, 0}^{\theta^{T^{\prime}}} s_{l, 1}^{\theta^{T^{\prime}}}
$$

hold.

By the last part of the proof of lemma 2.14, if the union of the walls separating $\Theta\left(V^{\theta^{T}}(y)\right), \Theta\left(V^{\theta^{T^{\prime}}}(y)\right)$ and $\Theta\left(V^{\theta^{T^{\prime}}}(y)\right), \Theta\left(V^{\theta^{T^{\prime \prime}}}(y)\right)$ are exactly the walls that separate $\Theta\left(V^{\theta^{T}}(y)\right), \Theta\left(V^{\theta^{T^{\prime \prime}}}(y)\right)$ and if $V^{\theta^{T}}, V^{\theta^{T^{\prime}}}$ and $V^{\theta^{T^{\prime}}}, V^{\theta^{T^{\prime \prime}}}$ in a neighbourhood of $y$ are related by equations of the form (18), then so are $V^{\theta^{T}}, V^{\theta^{T^{\prime \prime}}}$. It follows that we can restrict to equations (18) for which $\Theta\left(V^{\theta^{T}}(y)\right), \Theta\left(V^{\theta^{T^{\prime}}}(y)\right)$ either coincide or are separated by a wall $W_{\left\{J, J^{\complement}\right\}}=\Theta\left(V^{\theta^{T}}(y)\right) \cap \Theta\left(V^{\theta^{T^{\prime}}}(y)\right)$. 
We claim that we can restrict this set of equations further to the set of equations for $T, T^{\prime}$ with $\left|T \cap T^{\prime}\right|=2$. Given $T, T^{\prime}$ with $\left|T \cap T^{\prime}\right|<2$ we show that we can replace $T$ by $\tilde{T}$ (and similarly $T^{\prime}$ by $\tilde{T}^{\prime}$ ), possibly multiple times, such that in each step $\theta^{\tilde{T}} \in \Theta\left(V^{\theta^{T}}(y)\right),|T \cap \tilde{T}|=2$ and finally $\left|\tilde{T} \cap T^{\prime}\right|=2$. As the case that $\Theta\left(V^{\theta^{T}}(y)\right)$ and $\Theta\left(V^{\theta^{T^{\prime}}}(y)\right)$ coincide is trivial, we can assume $\Theta\left(V^{\theta^{T}}(y)\right) \cap \Theta\left(V^{\theta^{T^{\prime}}}(y)\right)=W_{\left\{J, J^{\mathrm{C}}\right\}}$ for some $J$, so that $\left(s_{i}^{\theta^{T}}(y)\right)_{i \in J^{\mathrm{C}}}$ coincide and $\left(s_{j}^{\theta^{T^{\prime}}}(y)\right)_{j \in J}$ coincide. If $T \subset J$ then there is $\tilde{T}$ such that $\theta^{\tilde{T}} \in \Theta\left(V^{\theta^{T}}(y)\right),|T \cap \tilde{T}|=2, \tilde{T} \cap J^{\complement} \neq \varnothing$, thus we can assume that both $T$ and $T^{\prime}$ have nonempty intersection with $J$ and $J^{\complement}$. If $T \cap T^{\prime}=\varnothing$ there exists $\tilde{T}$ such that $\theta^{\tilde{T}} \in \Theta\left(V^{\theta^{T}}(y)\right),|T \cap \tilde{T}|=2,\left|\tilde{T} \cap T^{\prime}\right|=1$ : to define $\tilde{T}=\left\{i_{1}, i_{2}, i_{3}\right\}$ let $i_{1} \in T^{\prime} \cap J^{\complement}$ and then $i_{2}, i_{3} \in T$ such that $s_{i_{1}}^{\theta^{T}}(y) \neq s_{i_{2}}^{\theta^{T}}(y), s_{i_{3}}^{\theta^{T}}(y)$. If $\left|T \cap T^{\prime}\right|=1$ there is $\tilde{T}$ such that $\theta^{\tilde{T}} \in \Theta\left(V^{\theta^{T}}(y)\right),|T \cap \tilde{T}|=2=\left|\tilde{T} \cap T^{\prime}\right|$ : to define $\tilde{T}=\left\{i_{1}, i_{2}, i_{3}\right\}$ let $\left\{i_{1}\right\}=T \cap T^{\prime}$, then $i_{2} \in T^{\prime} \cap J$ if $i_{1} \in J^{\complement}$ and $i_{2} \in T^{\prime} \cap J^{\complement}$ if $i_{1} \in J$, and then $i_{3} \in T$ such that $s_{i_{3}}^{\theta^{T}}(y) \neq s_{i_{1}}^{\theta^{T}}(y), s_{i_{2}}^{\theta^{T}}(y)$.

Having shown that in the functor $\operatorname{Lim}^{\prime}\left(Q_{n}\right)$ the equations (18) with $\left|T \cap T^{\prime}\right|=2$ are sufficient, consider $T=\left\{i_{1}, i_{2}, i_{3}\right\}, T^{\prime}=\left\{i_{1}, i_{2}, i_{4}\right\}$. By remark 2.17 we can restrict to the equations with $i=i_{1}, j=i_{2}$, further the equations with $k=i_{4}$ suffice, and we write $i_{5}$ for $l$. There are two cases $i_{3}=i_{5}$ and $i_{3} \neq i_{5}$ giving rise to two sets of equations. This shows that $\operatorname{Lim}^{\prime}\left(Q_{n}\right)$ is isomorphic to the functor $\operatorname{Lim}^{\prime \prime}\left(Q_{n}\right)$ defined by

$$
\begin{aligned}
& Y \mapsto \\
& \left\{\begin{array}{l|l}
\left(\varphi_{V^{\theta^{T}}}\right)_{T} & \begin{array}{l}
\forall i_{1}, i_{2}, i_{3}, i_{4} \text { pairwise distinct: } \\
s_{i_{4}, 0}^{\left\{i_{1}, i_{2}, i_{3}\right\}} s_{i_{3}, 1}^{\left\{i_{1}, i_{2}, i_{3}\right\}} s_{i_{4}, 1}^{\left\{i_{1}, i_{2}, i_{4}\right\}} s_{i_{3}, 0}^{\left\{i_{1}, i_{2}, i_{4}\right\}}=s_{i_{4}, 1}^{\left\{i_{1}, i_{2}, i_{3}\right\}} s_{i_{3}, 0}^{\left\{i_{1}, i_{2}, i_{3}\right\}} s_{i_{4}, 0}^{\left\{i_{1}, i_{2}, i_{4}\right\}} s_{i_{3}, 1}^{\left\{i_{1}, i_{2}, i_{4}\right\}} \\
\forall i_{1}, i_{2}, i_{3}, i_{4}, i_{5} \text { pairwise distinct: } \\
s_{i_{4}, 0}^{\left\{i_{1}, i_{2}, i_{3}\right\}} s_{i_{5}, 1}^{\left\{i_{1}, i_{2}, i_{3}\right\}} s_{i_{4}, 1}^{\left\{i_{1}, i_{2}, i_{4}\right\}} s_{i_{5}, 0}^{\left\{i_{1}, i_{2}, i_{4}\right\}}=s_{i_{4}, 1}^{\left\{i_{1}, i_{2}, i_{3}\right\}} s_{i_{5}, 0}^{\left\{i_{1}, i_{2}, i_{3}\right\}} s_{i_{4}, 0}^{\left\{i_{1}, i_{2}, i_{4}\right\}} s_{i_{5}, 1}^{\left\{i_{1}, i_{2}, i_{4}\right\}}
\end{array}
\end{array}\right\}
\end{aligned}
$$

where we choose coordinates such that $s_{i_{1}}^{\theta^{\left\{i_{1}, i_{2}, i_{3}\right\}}}, s_{i_{1}}^{\theta^{\left\{i_{1}, i_{2}, i_{4}\right\}}}=(0: 1), s_{i_{2}}^{\theta^{\left\{i_{1}, i_{2}, i_{3}\right\}}}$, $s_{i_{2}}^{\theta^{\left\{i_{1}, i_{2}, i_{4}\right\}}}=(1: 0)$ and use the abbreviations of the form $s^{\left\{i_{1}, i_{2}, i_{3}\right\}}=s^{\theta^{\left\{i_{1}, i_{2}, i_{3}\right\}}}$.

Each $V^{\theta^{T}}$ defining an element $\mathcal{M}^{\theta^{T}}\left(Q_{n}\right)(Y)$ consists of a family $\left(s_{k}^{\theta^{T}}\right)_{k=1, \ldots, n}$ of sections $s_{k}^{\theta^{T}}: Y \rightarrow \mathbb{P}_{Y}^{1}$ up to operation of $\mathrm{PGL}(2)$ on $\mathbb{P}_{Y}^{1}$. Within its isomorphism class, if $T=\left\{i_{1}, i_{2}, i_{3}\right\}$, we can choose this family such that $s_{i_{1}}^{\theta^{T}}=(0: 1), s_{i_{2}}^{\theta^{T}}=(1: 0)$, $s_{i_{3}}^{\theta^{T}}=(1: 1)$, then we denote $s_{i_{4}}^{i_{1}, i_{2}, i_{3}}=s_{i_{4}}^{\theta^{T}}: Y \rightarrow \mathbb{P}_{Y}^{1}$. The 3 ! sections corresponding to $s_{i_{4}}^{\theta^{T}}$ for $T=\left\{i_{1}, i_{2}, i_{3}\right\}$ are related by equations (15). The first set of equations of $\operatorname{Lim}^{\prime \prime}\left(Q_{n}\right)$ gives equations (16). The second set of equations of $\operatorname{Lim}^{\prime \prime}\left(Q_{n}\right)$ gives the equations of the form

$$
s_{i_{4}, 0}^{i_{1}, i_{2}, i_{3}} s_{i_{5}, 1}^{i_{1}, i_{2}, i_{3}} s_{i_{5}, 0}^{i_{1}, i_{2}, i_{4}}=s_{i_{4}, 1}^{i_{1}, i_{2}, i_{3}} s_{i_{5}, 0}^{i_{1}, i_{2}, i_{3}} s_{i_{5}, 1}^{i_{1}, i_{2}, i_{4}}
$$

The functor that associates to an $S$-scheme $Y$ families of sections $s_{i_{4}}^{i_{1}, i_{2}, i_{3}}: Y \rightarrow \mathbb{P}_{Y}^{1}$ for $i_{1}, i_{2}, i_{3}, i_{4} \in\{1, \ldots, n\},\left|\left\{i_{1}, i_{2}, i_{3}\right\}\right|=3$ that satisfy these equations is functor (17).

\section{Hassett moduli SPACES AND MODUli SPACES OF QUiVER REPRESENTATIONS}

4.1. Hassett moduli spaces $\bar{M}_{0, a}$ OF Weighted POinted CuRves of Genus ZERo. The Hassett moduli spaces $\bar{M}_{g, a}$ of $a$-stable $n$-pointed curves of genus $g$ for a weight $a$ were introduced in [15]. Here we consider the Hassett moduli spaces $\bar{M}_{0, a}$ of 
curves of genus 0 . The weight $a=\left(a_{1}, \ldots, a_{n}\right) \in \mathbb{Q}^{n}$ satisfies $0<a_{i} \leqslant 1$ for all $i$ and $|a|=\sum_{i} a_{i}>2$.

DeFINITION 4.1. An a-stable n-pointed curve of genus 0 over an algebraically closed field is a tuple $\left(C, s_{1}, \ldots, s_{n}\right)$ where $C$ is a complete connected reduced curve $C$ of genus 0 with at most ordinary double points (i.e. a tree of $\mathbb{P}^{1}$ 's), $s_{1}, \ldots, s_{n}$ are closed points of $C$ such that each $s_{i}$ is a regular point of $C$, if $\left(s_{i}\right)_{i \in I}$ coincide then $\sum_{i \in I} a_{i} \leqslant 1$, and the $\mathbb{Q}$-divisor $K_{C}+a_{1} s_{1}+\ldots+a_{n} s_{n}$ is ample, i.e. for each irreducible component $C^{k}$ of $C$ we have (number of intersection points with other components) $+\sum_{s_{i} \in C^{k}} a_{i}>2$.

Definition - Theorem 4.2 ([15]). The Hassett moduli space $\bar{M}_{0, a}$ is the fine moduli space of a-stable n-pointed curves of genus 0 , i.e. $\bar{M}_{0, a}$ represents the moduli functor (denoted by the same symbol)

$$
Y \mapsto\{\text { isomorphism classes of a-stable n-pointed curves of genus } 0 \text { over } Y\}
$$

where an a-stable n-pointed curve of genus 0 over a scheme $Y$ is a flat proper morphism $C \rightarrow Y$ with sections $s_{1}, \ldots, s_{n}: Y \rightarrow C$ such that the geometric fibres $\left(C_{y}, s_{1}(y), \ldots, s_{n}(y)\right)$ are a-stable n-pointed curves of genus 0 .

For $a=(1, \ldots, 1)$ the Hassett moduli space $\bar{M}_{0, a}$ coincides with the GrothendieckKnudsen moduli space $\bar{M}_{0, n}$, for $a=(1,1, \varepsilon, \ldots, \varepsilon), 0<\varepsilon \ll 1$ the moduli space $\bar{M}_{0, a}$ coincides with the Losev-Manin moduli space $\bar{L}_{n}$ (cf. [15, 6.4 and 7.1]).

4.2. The Functor of POINTS OF $\bar{M}_{0, a}$. We give a description of the functor of $\bar{M}_{0, a}$ similar to theorem 3.11 and 3.14 based on natural embeddings of $a$-stable $n$-pointed curves of genus 0 in a product of $\mathbb{P}^{1}$ 's.

Let $\left(C \rightarrow Y, s_{1}, \ldots, s_{n}\right)$ be an $a$-stable $n$-pointed curve of genus 0 over $Y$. Using again the methods of $[21]$, for $T \subseteq\{1, \ldots, n\},|T|=3$ the sections $\left(s_{i}\right)_{i \in T}$ define a contraction morphism to a $\mathbb{P}^{1}$-bundle over $Y$. Restricting to the open subscheme $Y^{T} \subseteq Y$ where $\left(s_{i}\right)_{i \in T}$ are pairwise disjoint, we obtain a trivial $\mathbb{P}^{1}$-bundle $\mathbb{P}_{Y^{T}}^{1} \rightarrow Y^{T}$ with sections $s_{1}^{T}, \ldots, s_{n}^{T}$ such that $\left(s_{i}^{T}\right)_{i \in T}$ are pairwise disjoint. As in example 3.12 we can introduce homogeneous coordinates $x_{0}^{i_{1}, i_{2}, i_{3}}, x_{1}^{i_{1}, i_{2}, i_{3}}$ on $\mathbb{P}_{Y^{T}}^{1}$ such that $s_{i_{1}}^{T}=(0$ : 1), $s_{i_{2}}^{T}=(1: 0), s_{i_{3}}^{T}=(1: 1)$. We write the sections $s_{j}^{T}$ respect to these coordinates as $s_{j}^{i_{1}, i_{2}, i_{3}}=\left(s_{j, 0}^{i_{1}, i_{2}, i_{3}}: s_{j, 1}^{i_{1}, i_{2}, i_{3}}\right)$.

Under permutations of the elements of $T$ these sections are related by the formulae (15). For $I=\left\{i_{1}, i_{2}, i_{3}, i_{4}\right\} \subseteq\{1, \ldots, n\},|I|=4$ and $T, T^{\prime} \subset I, T=\left\{i_{1}, i_{2}, i_{3}\right\}$, $T^{\prime}=\left\{i_{1}, i_{2}, i_{4}\right\}$ over $Y^{T} \cap Y^{T^{\prime}}$ we have the relation (16). Let $J=\left\{i_{1}, i_{2}, i_{3}, i_{4}, i_{5}\right\} \subseteq$ $\{1, \ldots, n\},|J|=5$ and $I=\left\{i_{1}, i_{2}, i_{3}, i_{4}\right\}, I^{\prime}=\left\{i_{1}, i_{2}, i_{3}, i_{5}\right\}, I^{\prime \prime}=\left\{i_{1}, i_{2}, i_{4}, i_{5}\right\}$, $T=\left\{i_{1}, i_{2}, i_{3}\right\}, T^{\prime}=\left\{i_{1}, i_{2}, i_{4}\right\}$. Then over $Y^{T} \cap Y^{T^{\prime}}$ we have the relation

$$
s_{i_{4}, 0}^{i_{1}, i_{2}, i_{3}} s_{i_{5}, 1}^{i_{1}, i_{2}, i_{3}} s_{i_{5}, 0}^{i_{1}, i_{2}, i_{4}}=s_{i_{4}, 1}^{i_{1}, i_{2}, i_{3}} s_{i_{5}, 0}^{i_{1}, i_{2}, i_{3}} s_{i_{5}, 1}^{i_{1}, i_{2}, i_{4}}
$$

because over the open set $\left\{y \mid s_{i_{3}}(y) \neq s_{i_{4}}(y)\right\} \subseteq Y^{T} \cap Y^{T^{\prime}}$ we have the same situation as in the case $\bar{M}_{0, n}$ (see theorem 3.14), over $\left\{y \mid s_{i_{3}}(y), s_{i_{4}}(y)\right.$ in same component $\} \subseteq$ $Y^{T} \cap Y^{T^{\prime}}$ these equations correspond to usual base changes in $\mathbb{P}^{1}$. 
THEOREM 4.3. The functor of $\bar{M}_{0, a}$ is isomorphic to the contravariant functor

$$
\begin{aligned}
& Y \mapsto \\
& \left\{\begin{array}{l|l} 
& (0) \forall T=\left\{i_{1}, i_{2}, i_{3}\right\},|T|=3: \\
& s_{i_{1}}^{i_{1}, i_{2}, i_{3}}=(0: 1), s_{i_{2}}^{i_{1}, i_{2}, i_{3}}=(1: 0), s_{i_{3}}^{i_{1}, i_{2}, i_{3}}=(1: 1) \\
(1) \forall I=\left\{i_{1}, i_{2}, i_{3}, i_{4}\right\},|I|=4: & \\
\text { families of sections } & \text { equations }(15) \text { and }(16) \text { hold } \\
s_{i_{4}, i_{2}, i_{3}}^{i_{1}}: Y^{T} \rightarrow \mathbb{P}_{Y^{T}}^{1} & (2) \forall J=\left\{i_{1}, i_{2}, i_{3}, i_{4}, i_{5}\right\},|J|=5: \\
\text { for } i_{1}, i_{2}, i_{3}, i_{4} \in\{1, \ldots, n\}, & \text { equations }(19) \text { hold } \\
T=\left\{i_{1}, i_{2}, i_{3}\right\},|T|=3, & (3) \forall T \forall y \in Y^{T}: w(y, T, a)>2 \\
Y^{T} \subseteq Y \text { open } & (4) \forall y \in Y \forall a^{\prime} \in \mathbb{Q}_{>0}^{n},\left|a^{\prime}\right|>2, a^{\prime} \leqslant a \exists T: \\
& y \in Y^{T}, w\left(y, T, a^{\prime}\right)>2 \\
& (5) \forall T, T^{\prime} \forall y \in Y^{T^{\prime}}: \\
& \left(s_{j}^{T^{\prime}}(y)\right)_{j \in T} \text { pairwise distinct } \Rightarrow y \in Y^{T}
\end{array}\right\}
\end{aligned}
$$

where sets $T$ (same for $T^{\prime}$ ) are always subsets $T \subseteq\{1, \ldots, n\}$ with $|T|=3$,

$$
w(y, T, a)=\sum_{l} \min \left\{1, \sum_{i \in J_{l}(y, T)} a_{i}\right\}
$$

for geometric points $y \in Y^{T}$ and the partitions $\{1, \ldots, n\}=\bigsqcup_{l} J_{l}(y, T)$ defined by $i, j \in J_{l}(y, T)$ for some $l$ if and only if $s_{i}^{T}(y)=s_{j}^{T}(y)$. We write $s_{i}^{T}$ if we work with some fixed ordering of $T$ that is not relevant.

Proof. We show that the above construction gives a morphism from $\bar{M}_{0, a}$ to the functor (20). Of the remaining conditions (3) is satisfied because of $a$-stability. Concerning condition (4), for an $a$-stable $n$-pointed tree $C$ over an algebraically closed field each intersection point $p$ of components divides the tree into two components, where for a given $a^{\prime} \in \mathbb{Q}_{>0}^{n},\left|a^{\prime}\right|>2, a^{\prime} \leqslant a$ the weights $a_{i}^{\prime}$ of the marked points of at least one component sum up to $>1$. Let $C^{p}$ be this component if it is unique, otherwise $C^{p}=C$. The intersection $\bigcap_{p} C^{p}$ is nonempty and $w\left(y, T, a^{\prime}\right)>2$ for each $T$ which singles out an irreducible component of $\bigcap_{p} C^{p}$. Condition (5) is satisfied because our construction defines $Y^{T}$ as the maximal set where the sections $\left(s_{i}\right)_{i \in T}$ are disjoint.

We define the morphism in the opposite direction: for an $S$-scheme $Y$ and a collection of sections $s_{i_{4}}^{i_{1}, i_{2}, i_{3}}: Y^{T} \rightarrow \mathbb{P}_{Y^{T}}^{1}$ as in (20) we construct an $a$-stable $n$ pointed tree over $Y$. For a geometric point $y \in Y$ let $\mathcal{T}^{y}=\left\{T \mid y \in Y^{T}\right\}$ and $U_{y}=\left\{y^{\prime} \in \bigcap_{T \in \mathcal{T}^{y}} Y^{T} \mid \forall T \in \mathcal{T}^{y}: s_{i}^{T}\left(y^{\prime}\right)=s_{j}^{T}\left(y^{\prime}\right) \Rightarrow s_{i}^{T}(y)=s_{j}^{T}(y)\right\}$. Define $C^{y} \subseteq \prod_{T \in \mathcal{T}^{y}}\left(\mathbb{P}_{Y^{T}}^{1}\right)_{U_{y}}$ as the subscheme defined by the equations

$$
s_{i_{4}, 0}^{i_{1}, i_{2}, i_{3}} x_{1}^{i_{1}, i_{2}, i_{3}} x_{0}^{i_{1}, i_{2}, i_{4}}=s_{i_{4}, 1}^{i_{1}, i_{2}, i_{3}} x_{0}^{i_{1}, i_{2}, i_{3}} x_{1}^{i_{1}, i_{2}, i_{4}}
$$

where $x_{0}^{i_{1}, i_{2}, i_{3}}, x_{1}^{i_{1}, i_{2}, i_{3}}$ are homogeneous coordinates of $\mathbb{P}_{Y^{T}}^{1}, T=\left\{i_{1}, i_{2}, i_{3}\right\}$, as in remark 3.15. We define sections $s_{i}^{y}=\left.\prod_{T \in \mathcal{T} y} s_{i}^{T}\right|_{U_{y}}: U_{y} \rightarrow C^{y} \subseteq \prod_{T \in \mathcal{T} y}\left(\mathbb{P}_{Y^{T}}^{1}\right)_{U_{y}}$ for $i \in\{1, \ldots, n\}$.

Let $I \subseteq\{1, \ldots, n\}$ such $s_{i}^{y}(y) \neq s_{i^{\prime}}^{y}(y)$ for $i, i^{\prime} \in I$ and for any $j \in\{1, \ldots, n\}$ there is $i \in I$ such that $s_{j}^{y}(y)=s_{i}^{y}(y)$. Then the same construction but restricted to $T \in \mathcal{T}^{y}$ with $T \subseteq I$ yields a curve $\bar{C}^{y} \subseteq\left(\prod_{T \in \mathcal{T}^{y}, T \subseteq I} \mathbb{P}_{Y^{T}}^{1}\right)_{U_{y}}$ with sections $\left(\bar{s}_{i}^{y}\right)_{i}$ over $U_{y}$ isomorphic to $\left(C^{y},\left(s_{i}^{y}\right)_{i}\right)$. By remark $3.15\left(\bar{C}^{y},\left(\bar{s}_{i}^{y}\right)_{i \in I}\right)$ is a stable $|I|$-pointed tree over $U_{y}$.

Using conditions (3) and (4) we show that the fibres $\left(C_{y}^{y},\left(s_{i}^{y}(y)\right)_{i}\right)$ are $a$ stable. The property that for each irreducible component $C_{y}^{y, k}$ of $C_{y}^{y}$ we have 
(number of intersection points with other components) $+\sum_{s_{i}^{y}(y) \in C_{y}^{y, k}} a_{i}>2$ follows from condition (3). The property that if $\left(s_{j}^{y}(y)\right)_{j \in J}$ coincide then $a(J)=\sum_{j \in J} a_{j} \leqslant 1$ follows from condition (4): if $\left(s_{j}^{y}(y)\right)_{j \in J}$ coincide, then for $0<\varepsilon<\min \{|a|-a(J),|a|-$ $2\}$ there is $a^{\prime}<a$ such that $a_{j}^{\prime}=a_{j}$ for $j \in J$ and $\sum_{i \in J^{0}} a_{i}^{\prime}=\max \{0,2-a(J)\}+\varepsilon$, by (4) there exists $T \in \mathcal{T}^{y}$ such that $w\left(y, T, a^{\prime}\right)>2$, and $a(J) \leqslant 1$ follows, because choosing $\varepsilon<\min \{1, a(J)-1\}$ in case $a(J)>1$ would imply $w\left(y, T, a^{\prime}\right)<2$.

Thus we have shown that $\left(C^{y},\left(s_{i}^{y}\right)_{i}\right)$ is an $a$-stable $n$-pointed tree over $U_{y}$.

We show that the data for $T \in \mathcal{T}^{y}$ are sufficient for constructing the pointed curve over $U_{y}$ : for $y^{\prime} \in U_{y}$ it is $\mathcal{T}^{y} \subseteq \mathcal{T}^{y^{\prime}}$ and we show that the projection $\prod_{T^{\prime} \in \mathcal{T} y^{\prime}}\left(\mathbb{P}_{Y^{T^{\prime}}}^{1}\right)_{U_{y} \cap U_{y^{\prime}}} \rightarrow \prod_{T \in \mathcal{T} y}\left(\mathbb{P}_{Y^{T}}^{1}\right)_{U_{y} \cap U_{y^{\prime}}}$ induces an isomorphism

$$
\left.\left.\left(C^{y^{\prime}},\left(s_{i}^{y^{\prime}}\right)_{i}\right)\right|_{U_{y} \cap U_{y^{\prime}}} \rightarrow\left(C^{y},\left(s_{i}^{y}\right)_{i}\right)\right|_{U_{y} \cap U_{y^{\prime}}} .
$$

A set $T^{\prime} \in T^{y^{\prime}}$ is not contained in $\mathcal{T}^{y}$ if and only if $\left(s_{i}^{y}(y)\right)_{i \in T^{\prime}}$ are not pairwise distinct. Assume $T^{\prime}=\left\{i_{1}, i_{2}, i_{3}^{\prime}\right\}$ such that $s_{i_{1}}^{y}(y) \neq s_{i_{2}}^{y}(y)=s_{i_{3}^{\prime}}^{y}(y)$. Let $T=\left\{i_{1}, i_{2}, i_{3}\right\} \in \mathcal{T}^{y}$ such that the projection of $C^{y}$ onto the factor $\left(\mathbb{P}_{Y^{T}}^{1}\right)_{U_{y}}$ corresponding to $T$ induces an isomorphism of the irreducible component of $C_{y}^{y}$ that contains $s_{i_{2}}^{y}$ and $\left(\mathbb{P}_{Y^{T}}^{1}\right)_{y}$. Then the coordinates for $T$ and $T^{\prime}$ are related over $U_{y} \cap U_{y^{\prime}}$ by $s_{i_{3}^{\prime}, 0}^{i_{1}, i_{2}, i_{3}} x_{1}^{i_{1}, i_{2}, i_{3}} x_{0}^{i_{1}, i_{2}, i_{3}^{\prime}}=$ $s_{i_{3}^{\prime}, 1}^{i_{1}, i_{2}, i_{3}} x_{0}^{i_{1}, i_{2}, i_{3}} x_{1}^{i_{1}, i_{2}, i_{3}^{\prime}}$ where $s_{i_{3}^{\prime}}^{i_{1}, i_{2}, i_{3}}$ does not meet $(0: 1),(1: 0)$ since $s_{i_{3}^{\prime}}^{T}$ does not meet $s_{i_{1}}^{T}, s_{i_{2}}^{T}$ over $U_{y^{\prime}}$. In the case that three sections $\left(s_{i}^{y}\right)_{i \in T^{\prime \prime}}, T^{\prime \prime} \in \mathcal{T}^{y^{\prime}}$, coincide over $y$, there is a $T^{\prime} \in \mathcal{T}^{y^{\prime}}$ with $\left|T^{\prime} \cap T^{\prime \prime}\right|=2$ such that the coordinates for $T^{\prime \prime}$ and $T^{\prime}$ are related in a similar way and only two of the sections $\left(s_{i}^{y}(y)\right)_{i \in T^{\prime}}$ coincide.

It follows that the $a$-stable $n$-pointed trees $\left(C^{y} \rightarrow U_{y},\left(s_{i}^{y}\right)_{i}\right)$ glue to an $a$-stable $n$-pointed tree $\left(C \rightarrow Y,\left(s_{i}\right)_{i}\right)$ over $Y$. This construction defines a morphism from the functor (20) to $\bar{M}_{0, a}$.

One verifies that these two morphisms are mutually inverse.

4.3. Hassett moduli SPACES AS LIMITS OF MOdULI SPACES OF QUIVER REPRESENTATiONS. In theorem 3.16 we have described the Losev-Manin moduli space as the limit over moduli spaces of quiver representations $\mathcal{M}^{\theta}\left(P_{n}\right)$. By corollary 2.8 this is the same as the limit over moduli spaces of representations of the quiver $Q_{n+2}$ for weights in a neighbourhood of a vertex of $\Delta(2, n+2)$ : we have $\bar{L}_{n}=\varliminf_{\leftarrow<a} \mathcal{M}^{\theta}\left(Q_{n+2}\right)$ for $a=(1,1, \varepsilon, \ldots, \varepsilon) \in \mathbb{Q}^{n+2}, 0<\varepsilon \ll 1$. On the other hand for the same weight $a$ we have $\bar{L}_{n} \cong \bar{M}_{0, a}$. We show that a similar statement relating Hassett moduli spaces of weighted $n$-pointed curves of genus zero to an inverse limit of moduli spaces of representations of $Q_{n}$ holds in general.

TheOREM 4.4. Let $a=\left(a_{1}, \ldots, a_{n}\right) \in \mathbb{Q}^{n}$ such that $0<a_{i} \leqslant 1,|a|=\sum_{i} a_{i}>2$. The Hassett moduli space $\bar{M}_{0, a}$ is the limit over the moduli spaces of representations $\mathcal{M}^{\theta}\left(Q_{n}\right)$ for $\theta \in P(a)=\{\theta \in \Delta(2, n) \mid \theta<a\}$, i.e.

$$
\bar{M}_{0, a} \cong \lim _{\dot{\theta}<a} \mathcal{M}^{\theta}\left(Q_{n}\right)
$$

Proof. We verify that the results in subsection 2.4 remain valid if we consider the limit over weights in the convex polytope $P(a)$ and obtain the following analogue of proposition 2.15: The functor $\lim _{\theta<a} \mathcal{M}^{\theta}\left(Q_{n}\right)$ is isomorphic to the functor $\operatorname{Lim}\left(Q_{n}, a\right)$ defined by

$$
Y \mapsto\left\{\left(\varphi_{V^{\theta}}\right)_{\theta} \in \prod_{\theta<a \text { generic }} \mathcal{M}^{\theta}(Q)(Y) \mid \text { locally equations (8) hold for }\left(V^{\theta}\right)_{\theta}\right\}
$$


where the product is over representatives $\theta$ of the generic GIT equivalence classes that meet $P(a)$ and $\varphi_{V^{\theta}}: Y \rightarrow \mathcal{M}^{\theta}(Q)$ is the morphism determined by a $\theta$-stable representation $V^{\theta}$ over $Y$.

As in the proof of theorem 3.17 there is a description in terms of the weights $\theta^{T}$, but one has to take into consideration that for some $\left(V^{\theta}\right)_{\theta}$ defining an element of $\operatorname{Lim}\left(Q_{n}, a\right)(Y)$ there might be points $y \in Y$ such that the polytopes $\Theta\left(V^{\theta}(y)\right)$ do not cover $\Delta(2, n)$ and leave out some of the $\theta^{T}$. So $\operatorname{Lim}\left(Q_{n}, a\right)$ is isomorphic to the functor $\operatorname{Lim}^{\prime}\left(Q_{n}, a\right)$ defined by

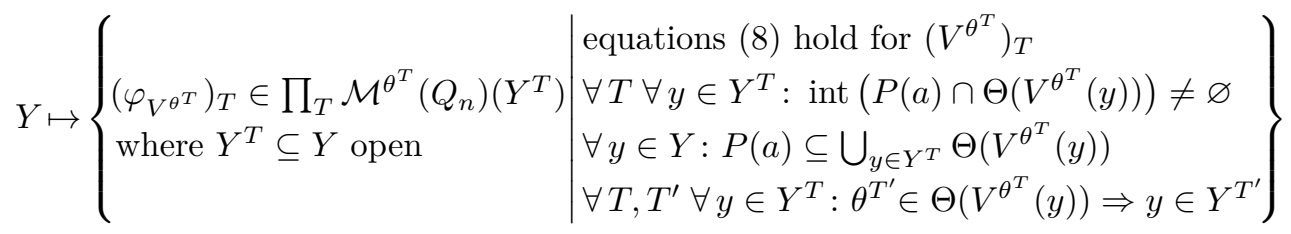

with the product over $T \subseteq\{1, \ldots, n\},|T|=3$.

As in the proof of theorem 3.17 the equations relating $V^{\theta^{T}}, V^{\theta^{T^{\prime}}}$ for $\left|T \cap T^{\prime}\right|=2$ are sufficient and we introduce the sections $s_{i_{4}}^{i_{1}, i_{2}, i_{3}}$. We obtain the functor $\operatorname{Lim}^{\prime \prime}\left(Q_{n}, a\right)$ defined by

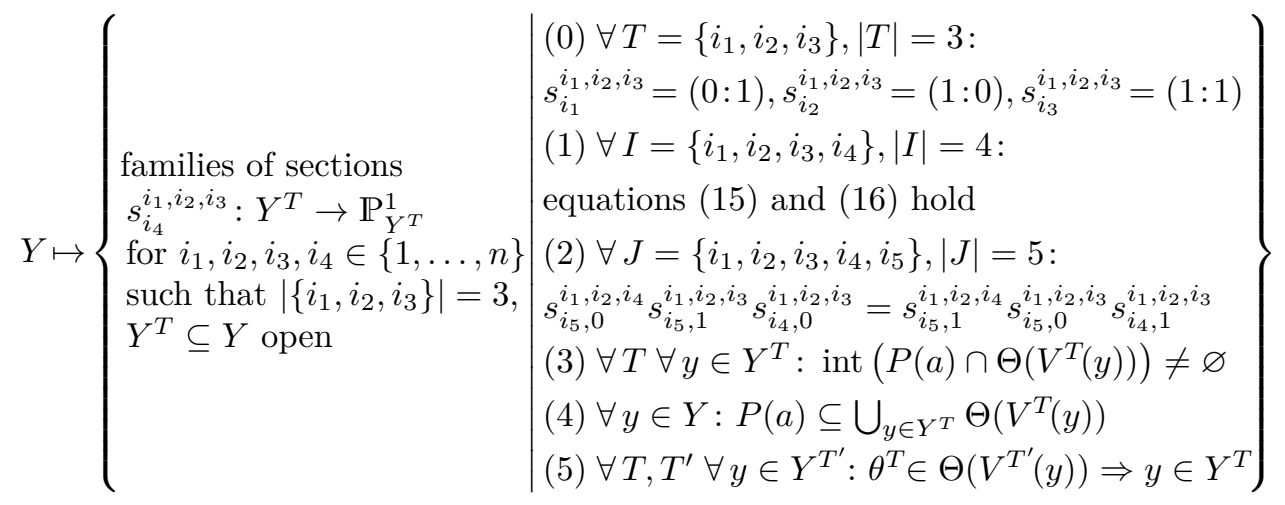

where we write $s_{j}^{T}$ for $s_{j}^{i_{1}, i_{2}, i_{3}}$ in case the order of elements of $T=\left\{i_{1}, i_{2}, i_{3}\right\}$ is irrelevant and $V^{T}$ for the representation (up to isomorphism) corresponding to $\left(s_{j}^{T}\right)_{j}$.

To show that this functor coincides with functor (20) we compare conditions $(3),(4),(5)$ in both. Let $\left(J_{l}(y, T)\right)_{l}$ and $w(y, T, a)$ be defined as in theorem 4.3, $\theta, a^{\prime}$ will always be elements of $\mathbb{Q}_{>0}^{n}$. Concerning condition(3) for a given $T$ and $y \in Y^{T}$ we have the equivalences

$$
\begin{aligned}
\operatorname{int}\left(P(a) \cap \Theta\left(V^{T}(y)\right)\right) \neq \varnothing & \Longleftrightarrow \exists \theta<a,|\theta|=2 \forall l: \sum_{i \in J_{l}(y, T)} \theta_{i}<1 \\
& \Longleftrightarrow \exists a^{\prime}<a: w\left(y, T, a^{\prime}\right)=\left|a^{\prime}\right|>2 \\
& \Longleftrightarrow w(y, T, a)>2 .
\end{aligned}
$$

Concerning condition (4) for fixed $y \in Y$ we have the equivalences

$$
\begin{aligned}
P(a) \subseteq \bigcup_{y \in Y^{T}} \Theta\left(V^{T}(y)\right) & \Longleftrightarrow \forall \theta<a,|\theta|=2 \exists T: y \in Y^{T}, V^{T}(y) \theta \text {-semistable } \\
& \Longleftrightarrow \forall \theta<a,|\theta|=2 \exists T: y \in Y^{T}, \forall l: \sum_{i \in J_{l}(y, T)} \theta_{i} \leqslant 1 \\
& \Longleftrightarrow \forall \theta<a,|\theta|=2 \exists T: y \in Y^{T}, w(y, T, \theta)=2 \\
& \Longleftrightarrow \forall a^{\prime} \leqslant a,\left|a^{\prime}\right|>2 \exists T: y \in Y^{T}, w\left(y, T, a^{\prime}\right)>2 .
\end{aligned}
$$


where for the implication " $\Longrightarrow$ " of the last equivalence use that for $a^{\prime} \leqslant a,\left|a^{\prime}\right|>2$ and $\theta \in \operatorname{int}\left(P\left(a^{\prime}\right)\right)$ we have $a^{\prime}=\theta+b$ with $b \in \mathbb{Q}_{>0}^{n}$. Concerning condition (5) we have $\theta^{T} \in \Theta\left(V^{T^{\prime}}(y)\right) \Longleftrightarrow V^{T^{\prime}}(y) \theta^{T}$-stable $\Longleftrightarrow\left(s_{j}^{T^{\prime}}(y)\right)_{j \in T}$ pairwise distinct.

Thus $\operatorname{Lim}^{\prime \prime}\left(Q_{n}, a\right)$ is isomorphic to the functor of $\bar{M}_{0, a}$ in (20).

REMARK 4.5. Our results fit well with the chamber decomposition in [15, section 5] and the observation in [15, section 8] that the corresponding GIT quotients can be interpreted as the moduli spaces $\bar{M}_{0, a}$ for $|a|=2$. In particular, we recover [15, Theorem 8.2, Theorem 8.3].

\section{REFERENCES}

[1] Jarod Alper, Adequate moduli spaces and geometrically reductive group schemes, Algebr. Geom. 1 (2014), no. 4, 489-531, https://arxiv.org/abs/1005.2398.

[2] Sivaramakrishna Anantharaman, Schémas en groupes, espaces homogènes et espaces algébriques sur une base de dimension 1, in Sur les groupes algébriques, Bull. Soc. Math. France, Mém., vol. 33, Soc. Math. France, 1973, pp. 5-79.

[3] Victor Batyrev and Mark Blume, The functor of toric varieties associated with Weyl chambers and Losev-Manin moduli spaces, Tohoku Math. J. (2) 63 (2011), no. 4, 581-604, https:// arxiv.org/abs/0911.3607.

[4] Sylvain Brochard, Topologies de Grothendieck, descente, quotients, in Autour des schémas en groupes. Vol. I, Panor. Synthèses, vol. 42/43, Soc. Math. France, Paris, 2014, https://arxiv. org/abs/1210.0431, pp. 1-62.

[5] Calin Chindris, Notes on GIT-fans for quivers, https://arxiv.org/abs/0805.1440.

[6] William Crawley-Boevey, Subrepresentations of general representations of quivers, Bull. London Math. Soc. 28 (1996), no. 4, 363-366.

[7] Igor V. Dolgachev and Yi Hu, Variation of geometric invariant theory quotients, Publ. Math. Inst. Hautes Étud. Sci. (1998), no. 87, 5-51, https://arxiv.org/abs/alg-geom/9402008.

[8] Israel M. Gel'fand, Mark Goresky, Robert D. MacPherson, and Vera V. Serganova, Combinatorial geometries, convex polyhedra, and Schubert cells, Adv. in Math. 63 (1987), no. 3, 301-316.

[9] Israel M. Gel'fand, Mikhail M. Kapranov, and Andrei V. Zelevinsky, Discriminants, resultants, and multidimensional determinants, Mathematics: Theory \& Applications, Birkhäuser Boston, Inc., Boston, MA, 1994

[10] Lothar Gerritzen, Frank Herrlich, and Marius van der Put, Stable n-pointed trees of projective lines, Nederl. Akad. Wetensch. Indag. Math. 50 (1988), no. 2, 131-163.

[11] Noah Giansiracusa, David Jensen, and Han-Bom Moon, GIT compactifications of $M_{0, n}$ and flips, Adv. Math. 248 (2013), 242-278, https://arxiv.org/abs/1112.0232.

[12] Alexander Grothendieck and Jean A. Dieudonné, Éléments de géométrie algébrique. I-IV, Publ. Math., Inst. Hautes Étud. Sci. 4, 8, 11, 17, 20, 24, 28, 32 (1960-1967).

[13]_ Éléments de géométrie algébrique. I, Grundlehren der Mathematischen Wissenschaften [Fundamental Principles of Mathematical Sciences], vol. 166, Springer-Verlag, Berlin, 1971.

[14] Alexander Grothendieck et al., Séminaire de géométrie algébrique, Groupes de monodromie en géométrie algébrique, Tome 1, Lecture Notes in Mathematics, vol. 288, Springer, 1972.

[15] Brendan Hassett, Moduli spaces of weighted pointed stable curves, Adv. Math. 173 (2003), no. 2, 316-352, https://arxiv.org/abs/math/0205009.

[16] Lutz Hille and José Antonio de la Peña, Stable representations of quivers, J. Pure Appl. Algebra 172 (2002), no. 2-3, 205-224.

[17] Mikhail M. Kapranov, Chow quotients of Grassmannians. I, in I. M. Gel'fand Seminar, Adv. Soviet Math., vol. 16, Amer. Math. Soc., Providence, RI, 1993, https://arxiv.org/abs/alg-geom/ 9210002, pp. 29-110.

[18] _ Veronese curves and Grothendieck-Knudsen moduli space $\bar{M}_{0, n}$, J. Algebraic Geom. 2 (1993), no. 2, 239-262.

[19] Mikhail M. Kapranov, Bernd Sturmfels, and Andrei V. Zelevinsky, Quotients of toric varieties, Math. Ann. 290 (1991), no. 4, 643-655.

[20] Alastair D. King, Moduli of representations of finite-dimensional algebras, Quart. J. Math. Oxford Ser. (2) 45 (1994), no. 180, 515-530.

[21] Finn F. Knudsen, The projectivity of the moduli space of stable curves. II. The stacks $M_{g, n}$, Math. Scand. 52 (1983), no. 2, 161-199.

[22] Daniel Lazard, Autour de la platitude, Bull. Soc. Math. France 97 (1969), 81-128. 
[23] Andrey A. Losev and Yuri I. Manin, New moduli spaces of pointed curves and pencils of flat connections, Michigan Math. J. 48 (2000), 443-472, https://arxiv.org/abs/math/0001003.

[24] David Mumford, John Fogarty, and Frances Kirwan, Geometric invariant theory, third ed., Ergebnisse der Mathematik und ihrer Grenzgebiete (2) [Results in Mathematics and Related Areas (2)], vol. 34, Springer-Verlag, Berlin, 1994.

[25] Nicolas Ressayre, The GIT-equivalence for G-line bundles, Geom. Dedicata 81 (2000), no. 1-3, 295-324, https://arxiv.org/abs/math/9811053.

[26] Aidan Schofield, General representations of quivers, Proc. London Math. Soc. (3) 65 (1992), no. $1,46-64$

[27] Jiro Sekiguchi, Cross ratio varieties for root systems, Kyushu J. Math. 48 (1994), no. 1, 123-168.

[28] _ Cross ratio varieties for root systems of type A and the Terada model, J. Math. Sci. Univ. Tokyo 3 (1996), no. 1, 181-197.

[29] Conjeeveram S. Seshadri, Geometric reductivity over arbitrary base, Advances in Math. 26 (1977), no. 3, 225-274.

[30] Stacks Project Authors, Stacks project, https://stacks.math.columbia.edu.

[31] Michael Thaddeus, Geometric invariant theory and flips, J. Amer. Math. Soc. 9 (1996), no. 3, 691-723, https://arxiv.org/abs/alg-geom/9405004.

[32] _ Complete collineations revisited, Math. Ann. 315 (1999), no. 3, 469-495, https:// arxiv.org/abs/math/9808114.

Mark Blume, Universität Münster, Mathematisches Institut, Einsteinstrasse 62, 48149 Münster, Germany

E-mail : mark.blume@uni-muenster.de

Lutz Hille, Universität Münster, Mathematisches Institut, Einsteinstrasse 62, 48149 Münster, Germany

E-mail : lutzhille@uni-muenster.de 